\title{
On the Density of Unnormalized Tamagawa Numbers of Orthogonal Groups I
}

\author{
By \\ Norihiko HAYASAKA* and Akihiko YUkIE**
}

\section{$\S 1$. Introduction}

This is the first part of a series of three papers. In this series of papers, we determine the density of unnormalized Tamagawa numbers of projective special orthogonal groups defined over a fixed number field.

Let $k$ and $\mathbb{A}$ be a number field and its ring of adèles. Throughout this series of papers

$$
G=\mathrm{GL}(1) \times \mathrm{GL}(n), V=\operatorname{Sym}^{2} \mathrm{Aff}^{n} .
$$

We regard $V$ as the space of quadratic forms in $n \geq 1$ variables. In these papers we mainly consider the case $n \geq 3$, but need to consider all positive integers $n \in \mathbb{Z}_{>0}$ for technical reasons. Let $V_{k}^{\text {ss }}=\left\{x \in V_{k} \mid \operatorname{det} x \neq 0\right\}$. For $x \in V_{k}^{\text {ss }}$, we define the special orthogonal group $\mathrm{SO}(x)$ in the well-known manner. We define $\mathrm{PSO}(x)$ to be $\mathrm{SO}(x)$ modulo its center, and call it the projective special orthogonal group of $x$. Then

$$
\operatorname{PSO}(x)= \begin{cases}\mathrm{SO}(x) & n \text { odd } \\ \mathrm{SO}(x) /\left\{ \pm I_{n}\right\} & n \text { even }\end{cases}
$$

We denote the set of $k$-isomorphism classes of algebraic groups over $k$ of the form $\operatorname{PSO}(x)$ by $S_{n}$. Then $S_{n}$ can be naturally identified with the set of $k$-isomorphism classes of algebraic groups over $k$ of the form $\mathrm{SO}(x)$.

\footnotetext{
Communicated by S. Mukai. Received April 10, 2007, Revised October 15, 2007.

2000 Mathematics Subject Classification(s): 11S90, 11R45.

Key words: Density theorem, prehomogeneous vector spaces, quadratic forms, Tamagawa numbers, local zeta functions.

The first author was partially supported by Teijin Kumura scholarship.

* Mathematical Institute, Tohoku University, Sendai 980-8578, Japan.

e-mail: sa3m26@math.tohoku.ac.jp

** Mathematical Institute, Tohoku University, Sendai 980-8578, Japan.

e-mail: yukie@math.tohoku.ac.jp
}

(C) 2008 Research Institute for Mathematical Sciences, Kyoto University. All rights reserved. 
In $\S 3$, we prove that the correspondence $G_{k} \backslash V_{k}^{\text {ss }} \ni x \mapsto \operatorname{PSO}(x) \in S_{n}$ is a bijective map. In $\S 5$, we define the discriminant $\Delta_{x} \in \mathbb{Z}_{>0}$ for $x \in V_{k}^{\mathrm{ss}}$. In $\S 8$, we define an invariant measure $d \widetilde{g}_{x}^{\prime \prime}$ on the adèlization $\operatorname{PSO}(x)_{\mathbb{A}}$ essentially using its Iwasawa decomposition. This $d \widetilde{g}_{x}^{\prime \prime}$ is not the classical Tamagawa measure on $\operatorname{PSO}(x)_{\mathbb{A}}$, which is defined using an invariant differential form defined over $k$.

The volume $\operatorname{vol}\left(\operatorname{PSO}(x)_{\mathbb{A}} / \operatorname{PSO}(x)_{k}\right)$ with respect to $d \widetilde{g}_{x}^{\prime \prime}$ is finite, and we call it the unnormalized Tamagawa number of $\operatorname{PSO}(x)$. This is an arithmetic invariant of some interest. For example, if $n=2$ then it can be described by the class number and the regulator of the quadratic extension of $k$ generated by the roots of $x$.

Our main theorems are Theorem 6.12 in Part II [9] and Theorem 5.9 in Part III [34]. Our results are over an arbitrary number field $k$, but we state them here assuming that $k=\mathbb{Q}$ for simplicity.

For convenience, we put $r=\left[\frac{n}{2}\right]$, i.e., $r=\frac{n-1}{2}(n$ odd $)$ and $r=\frac{n}{2}(n$ even). For a prime number $p$, we put

$$
\begin{aligned}
& E_{p}=1-\frac{3}{4} p^{-2}-\frac{1}{4} p^{-3}-p^{-r-1}+\frac{1}{2} p^{-r-2}+\frac{1}{2} p^{-r-3}+\frac{1}{4} p^{-2 r-2}-\frac{1}{4} p^{-2 r-3} \\
& E_{p}^{\prime}=1-p^{-2}-p^{-2 r-1}+p^{-2 r-2}+\frac{1}{4} p^{-3} \frac{\left(1-p^{-1}\right)^{2}\left(1-p^{-(r-1)}\right)\left(1-p^{-2 r}\right)}{1-p^{-2}}
\end{aligned}
$$

Let $\Gamma(s)$ be the classical gamma function. We put

$$
\Gamma_{\mathbb{R}}(s)=\pi^{-\frac{s}{2}} \Gamma\left(\frac{s}{2}\right), \Gamma_{\mathbb{C}}(s)=(2 \pi)^{1-s} \Gamma(s) .
$$

For $0 \leq \dot{\mathrm{i}} \leq r$, let $S_{n, \dot{\mathrm{i}}}$ be the subset of $S_{n}$ consisting of groups of the form $\operatorname{PSO}(x)$ where $x$ is a quadratic form with signature $(n-\dot{\mathbb{i}}, \dot{\mathbb{i}})$. Note that this implies that there are $n-\dot{\mathbf{i}}$ positive eigenvalues.

For the special case $k=\mathbb{Q}$, our main results can be formulated as follows.

Theorem 1.2. $\quad$ Suppose that $n=2 r+1 \geq 3$ is odd. Then

$$
\begin{aligned}
& \lim _{X \rightarrow \infty} X^{-\frac{n+1}{2}} \sum_{\substack{x, y \in S_{n, \mathrm{i}} \\
\Delta_{x} \Delta y<X}} \operatorname{vol}\left(\mathrm{SO}(x)_{\mathbb{A}} / \mathrm{SO}(x)_{\mathbb{Q}}\right) \operatorname{vol}\left(\mathrm{SO}(y)_{\mathbb{A}} / \mathrm{SO}(y)_{\mathbb{Q}}\right) \\
& =\frac{2^{-n+\dot{\mathrm{i}}(n-\dot{\mathrm{i}}+1)+2}}{n+1}\left(\prod_{1 \leq j \leq \dot{\mathrm{i}}} \Gamma_{\mathbb{R}}(j) \prod_{1 \leq j \leq n-\dot{\mathrm{i}}} \Gamma_{\mathbb{R}}(j) \prod_{1 \leq j \leq r} \zeta(2 j)\right)^{2} \prod_{p} E_{p} .
\end{aligned}
$$

Note that $\mathrm{SO}(x) \cong \operatorname{PSO}(x)$ if $n$ is odd. 
Theorem 1.3. Suppose that $n=2 r \geq 4$ is even. Then

$$
\begin{aligned}
& \lim _{X \rightarrow \infty} X^{-\frac{n+1}{2}} \sum_{\substack{x \in S_{n, \mathrm{i}} \\
\Delta_{x}<X}} \operatorname{vol}\left(\operatorname{PSO}(x)_{\mathbb{A}} / \operatorname{PSO}(x)_{\mathbb{Q}}\right) \\
& =\frac{2^{-n+\frac{\mathbf{i}(n-\hat{\mathrm{i}}+1)}{2}+2}}{n+1} \prod_{1 \leq j \leq \mathfrak{i}} \Gamma_{\mathbb{R}}(i) \prod_{1 \leq j \leq n-\mathbf{i}} \Gamma_{\mathbb{R}}(j) \prod_{1 \leq j \leq r} \zeta(2 j) \prod_{p} E_{p}^{\prime} .
\end{aligned}
$$

Since our work is a generalization of Datskovsky's work [3], our method works for $n=2$ also, and can prove the following known result of GoldfeldHoffstein [7]

Theorem 1.4 (Goldfeld-Hoffstein).

$$
\begin{gathered}
\lim _{X \rightarrow \infty} X^{-\frac{3}{2}} \sum_{\substack{[F: \mathbb{Q}]=2 \\
0<\Delta_{F} \leq X}} h_{F} R_{F}=\frac{\pi^{2}}{36} \prod_{p}\left(1-p^{-2}-p^{-3}+p^{-4}\right), \\
\lim _{X \rightarrow \infty} X^{-\frac{3}{2}} \sum_{\substack{[F: \mathbb{Q}]=2 \\
0<-\Delta_{F} \leq X}} h_{F}=\frac{\pi}{18} \prod_{p}\left(1-p^{-2}-p^{-3}+p^{-4}\right)
\end{gathered}
$$

where $h_{F}, R_{F}$ are the class number and the regulator of the quadratic field $F$ respectively.

Note that $1-p^{-2}-p^{-2 r-1}+p^{-2 r-2}=1-p^{-2}-p^{-3}+p^{-4}$ if $r=1$, which is the constant in the theorem of Goldfeld-Hoffstein. Also note that the theorem of Goldfeld-Hoffstein is stronger than Datskovsky's work (and hence our work also) in the sense that they obtained an error term estimate. This aspect on the error term is very difficult if one uses the zeta function method, but it is something which should eventually be achieved with the zeta function method also for even $n$.

For a nonzero integer $D$, let $h_{D}$ be the number of $\mathrm{SL}(2)_{\mathbb{Z}}$-equivalence classes of primitive integral binary quadratic forms with discriminant $D$. It is known that $h_{D}$ equals the narrow class number of the order of a quadratic field with discriminant $D$. If $D>0$ then one can define an analogue of the regulator for the above order, which we denote by $R_{D}$. It is very famous that Gauss conjectured that

$$
\sum_{0<-D<X} h_{D} \sim \frac{4 \pi}{21 \zeta(3)} X^{\frac{3}{2}} .
$$

The integral structure on the space of binary quadratic forms Gauss used was different from the integral structure used nowadays. If the integral structure 
of this paper (which is the same as that in Shintani [26], etc.,) is used, the constant $4 / 21$ must be replaced by $1 / 18$. If we use the integral structure of this paper, Gauss' conjecture for $D>0$ can be stated as follows:

$$
\sum_{0<D<X} h_{D} R_{D} \sim \frac{\pi^{2}}{18 \zeta(3)} X^{\frac{3}{2}} .
$$

Gauss' conjecture was proved by Lipschutz [18] for the imaginary case (i.e., for $D<0$ ). The real case was proved by Siegel [27]. Mertens [19], Vinogradov [29], Shintani [26] and Chamizo-Iwaniec [2] worked on the error term estimates for these cases. Shintani estimated the error term using the zeta function theory of prehomogeneous vector spaces. Siegel's result [27] contains the density theorem of equivalence classes of integral quadratic forms in $n \geq 2$ variables.

Gauss' conjecture was a conjecture essentially on integral equivalent classes of integral binary quadratic forms. One can naturally associate a quadratic field to a binary quadratic form. Then a natural question is whether or not $h_{D}$ is related to the class number of the quadratic field with discriminant $D$. The answer is yes in some sense. If $D$ is square-free then $h_{D}$ is indeed the narrow class number of the quadratic field with discriminant $D$. However, in Gauss' conjecture, $h_{D}$ 's for not necessarily square-free $D$ were counted. If $m$ is a nonzero integer and $D=m^{2} D^{\prime}$ then $h_{D}, R_{D}$ can be easily described by $h_{D^{\prime}}, R_{D^{\prime}}$ and $m$. So to get the density of $h_{k} R_{k}$ of quadratic fields, one has to filter out the above ambiguity.

This ambiguity was first removed by Goldfeld and Hoffstein in [7]. Goldfeld and Hoffstein used Eisenstein series of half integral weight to prove Theorem 1.4. Datskovsky gave another proof by using the zeta function theory of the prehomogeneous vector space (1.1) for the case $n=2$ in [3].

Theorems 1.2, 1.3 are density theorems on rational equivalence classes $G_{\mathbb{Q}} \backslash V_{\mathbb{Q}}^{\text {ss }}$ and so differs from Siegel's result in some sense. We obtain natural objects such as $\mathbb{Q}$-isomorphism classes of (projective) special orthogonal groups by considering $G_{\mathbb{Q}} \backslash V_{\mathbb{Q}}^{\text {ss }}$. What we do is to remove the ambiguity based on the difference between integral equivalence classes and rational equivalence classes.

Considering rational equivalence classes sometimes makes the consideration easier and sometimes more difficult. If there are not enough equivalence classes, the consideration becomes easier. This is the case for odd $n$. If there are still many equivalence classes, the consideration becomes more difficult, because it is difficult to count sparse objects. This is the case for even $n$. For this reason, we use different methods for odd $n$ and even $n$.

The notion of prehomogeneous vector spaces was introduced by M. Sato in 
the early 1960's. The pair (1.1) is a typical example of prehomogeneous vector spaces. The principal parts of the global zeta functions for some prehomogeneous vector spaces, including (1.1), were determined by Shintani [25], [26] and Yukie [35], [36], [37]. Ibukiyama-Saito [11] proved an "explicit formula" for the zeta function for (1.1) when the ground field is $\mathbb{Q}$. They expressed the zeta function as a sum of two functions which are products of Riemann zeta functions in the case where $n$ is odd, and expressed the zeta function using Riemann zeta functions and the Eisenstein series of half integral weight in the case where $n$ is even.

For the rest of this introduction, we consider (1.1) over an arbitrary number field $k$. The main purpose of Parts I, II is to prove Theorem 1.2. For this purpose, we use a Dirichlet series $\widetilde{Z}(s)$ defined by

$$
\widetilde{Z}(s)=\sum_{x \in G_{k} \backslash V_{k}^{\mathrm{ss}}} \frac{\operatorname{vol}\left(\mathrm{SO}(x)_{\mathbb{A}} / \mathrm{SO}(x)_{k}\right)}{\Delta_{x}^{s}}
$$

when $n$ is odd. This $\widetilde{Z}(s)$ is not the zeta function of the prehomogeneous vector space (1.1). In Part II, we shall express $\widetilde{Z}(s)$ as a sum of two Euler products by a technique used in [11], and prove that $\widetilde{Z}(s)^{2}$ has the rightmost pole at $s=\frac{n+1}{2}$ which is simple. Then the well-known Tauberian theorem (see Theorem I [21, p.464]) reduces the problem to the computation of the residue of $\widetilde{Z}(s)^{2}$ at $s=\frac{n+1}{2}$. The slightly complicated form of Theorem 1.2 is a reflexion of the fact that $\widetilde{Z}(s)^{2}$, rather than $\widetilde{Z}(s)$, has a simple pole at the rightmost pole. The location of the poles of $\widetilde{Z}(s)^{2}$ for $\operatorname{Re}(s)<\frac{n+1}{2}$ is related to the generalized Riemann hypothesis. So it seems difficult to obtain any error term estimate. Even though we shall not prove it, we expect that

$$
X^{\frac{n+1}{2}-\epsilon} \ll \sum_{\substack{x \in S_{n} \\ \Delta x<X}} \operatorname{vol}\left(\mathrm{SO}(x)_{\mathbb{A}} / \mathrm{SO}(x)_{k}\right) \ll X^{\frac{n+1}{2}}
$$

for any $\epsilon>0$ if $n$ is odd.

For odd $n, \operatorname{vol}\left(\operatorname{SO}(x)_{\mathbb{A}} / \mathrm{SO}(x)_{k}\right)$ can be expressed as $2 \prod_{v} \widetilde{c}_{v, x}^{\prime \prime}$, where 2 is the value of the classical Tamagawa number of $\mathrm{SO}(x)$ and $\widetilde{c}_{v, x}^{\prime \prime}$ is a certain Euler factor corresponding to the place $v$ of $k$. If $v$ is a finite place then it turns out that the computation of $\widetilde{c}_{v, x}^{\prime \prime}$ reduces to the computation of the "local density" of $x$. If $k=\mathbb{Q}$ then the local density is known for all cases (see [8], [30]). However, there is a slight difficulty dealing with arbitrary dyadic fields and so we use a method similar to that in [3], [15] to compute $\widetilde{c}_{v, x}^{\prime \prime}$ for $v \in \mathfrak{M}_{\mathrm{f}}$ in $\S 11$. For even $n$, we shall group local orbits according to their types and compute the sum of $\widetilde{c}_{v, x}^{\prime \prime}$ for each type for $v \in \mathfrak{M}_{\mathrm{f}}$ in Part III. We shall compute $\widetilde{c}_{v, x}^{\prime \prime}$ for 
infinite places (including the case where $n$ is even) in Part II. The knowledge of $\widetilde{c}_{v, x}^{\prime \prime}$ for all $v$ and $x$ and the relatively simple orbit space $G_{k} \backslash V_{k}^{\text {ss }}$ enables us to use a technique in [11] to $\widetilde{Z}(s)$. We shall discuss the method for odd $n$ in the introduction of Part II in more detail (also see the comment at the end of $\S 6)$.

We shall prove Theorem 1.3 in Part III. We use the "filtering process" used in [4], [3], [14], [15], [16] (also implicitly in [5], [6]) for that purpose. This approach is based on the zeta function theory of the prehomogeneous vector space (1.1). Roughly speaking, the zeta function $Z(s)$ for this case is in the following form:

$$
Z(s)=\sum_{x \in G_{k} \backslash V_{k}^{\mathrm{ss}}} \frac{\operatorname{vol}\left(\mathrm{SO}(x)_{\mathbb{A}} / \mathrm{SO}(x)_{k}\right)}{\Delta_{x}^{s}} L_{x}(s)
$$

where $L_{x}(s)$ is a certain $L$-function which depends on the orbit $x \in V_{k}^{\text {ss }}$. So, in a sense, we use the filtering process to remove the contribution from $L_{x}(s)$.

We speculate that it is possible to use the filtering process to the square of the zeta function and obtain the same result for odd $n$. However, it is probably easier to apply the explicit method in Part II. There is also a possibility that one can use the explicit method in Part II for even $n$. However, since the principal parts of the zeta function for the present case has been determined in [35], it is probably easier to use the zeta function theory at this point. We discuss the method for even $n$ in the introduction of Part III in more detail.

For the rest of the introduction, we discuss the organization of this part. Except for $\S 3$ where $k$ is an arbitrary field, $k$ is a number field. In this part $n$ is an arbitrary positive integer except for $\S 6,9,10,11$ where $n \geq 3$ is an odd integer.

In $\S 2$, we discuss notations used throughout this part. In $\S 3$, we investigate the relation between $S_{n}$ and the orbit space $G_{k} \backslash V_{k}^{\text {ss }}$ for an arbitrary field $k$. In $\S 4$, we choose a set of representatives for local orbit spaces of (1.1) at finite places. In $\S 5$, we define the notion of discriminant for quadratic forms, and determine values of discriminants for the local representatives which we choose in $\S 4$. In $\S 6$, we investigate the correspondence between the global orbit space and the product of the local orbit spaces for odd $n$. In $\S 7$ and 8 , we define invariant measures on $\mathrm{SO}(x)_{\mathbb{A}}$, etc., for $x \in V_{k}^{\text {ss }}$ essentially using their Iwasawa decompositions, and define the notion of the unnormalized Tamagawa number of $\mathrm{SO}(x)_{\mathbb{A}}$, etc., assuming the definition of the measures at infinite places in Part II. In this way, the reader can concentrate on finite places in this part, and on infinite places in Part II. In $\S 9$, we review some facts concerning the classical Tamagawa number of $\mathrm{SO}(x)$. In $\S 10$ and 11 , we compute $\widetilde{c}_{v, x}^{\prime \prime}$ for finite 
places $v$. Part of the computations of $\widetilde{c}_{v, x}^{\prime \prime}$ may follow from classical results, but we included them for the sake of the reader.

\section{$\S 2 . \quad$ Notation}

In this section, we define basic notations used throughout this paper. More specialized notations will be introduced in each section.

If $X$ is a finite set then $\sharp X$ will denote its cardinality. The symbols $\mathbb{Q}, \mathbb{R}$, $\mathbb{C}$ and $\mathbb{Z}$ will denote respectively the set of rational, real and complex numbers and the rational integers. If $a \in \mathbb{R}$, then $[a]$ will denote the largest integer $z$ such that $z \leq a$. The symbol $\mathbb{R}_{>0}$ (resp. $\mathbb{R}_{\geq 0}$ ) will denote the set of positive (resp. non-negative) real numbers. Similarly, $\mathbb{Z}_{>0}\left(\right.$ resp. $\left.\mathbb{Z}_{\geq 0}\right)$ will denote the set of positive (resp. non-negative) integers. If $R$ is any ring, then $R^{\times}$is the set of invertible elements of $R$. If $V$ is a variety defined over $R$, then $V_{R}$ denotes the set of $R$-points. If $G$ is an algebraic group, then $G^{\circ}$ denotes its identity component.

In this paper, we assume that $k$ is a number field except for $\S 3$ where $k$ is an arbitrary field. We shall denote the ring of integers of $k$ by $\mathcal{O}$. The symbols $\mathfrak{M}, \mathfrak{M}_{\infty}, \mathfrak{M}_{\mathrm{f}}, \mathfrak{M}_{\mathrm{dy}}, \mathfrak{M}_{\mathbb{R}}$ and $\mathfrak{M}_{\mathbb{C}}$ will denote respectively the set of all places of $k$, all infinite places, all finite places, all dyadic places (those dividing the place of $\mathbb{Q}$ at 2 ), all real places and all imaginary places.

If $v \in \mathfrak{M}, k_{v}$ denotes the completion of $k$ at $v$ and ||$_{v}$ the normalized absolute value on $k_{v}$. If $v \in \mathfrak{M}_{\mathrm{f}}$, then $\mathcal{O}_{v}$ denotes the ring of integers of $k_{v}$, $\pi_{v}$ a uniformizer of $\mathcal{O}_{v}, \mathfrak{p}_{v}$ the maximal ideal of $\mathcal{O}_{v}$ and $q_{v}$ the cardinality of $\mathcal{O}_{v} / \mathfrak{p}_{v}$. If $a \in k_{v}$ and $(a)=\mathfrak{p}_{v}^{i}$, then we write $\operatorname{ord}_{v}(a)=i(\operatorname{or} \operatorname{ord}(a)=i$ if there is no confusion). If $\mathfrak{i}$ is a fractional ideal in $k_{v}$ and $a-b \in \mathfrak{i}$, then we write $a \equiv b(\mathfrak{i})$ or $a \equiv b(c)$ if $c$ generates $\mathfrak{i}$.

If $k_{1} / k_{2}$ is a finite extension either of local fields or of number fields, then we denote the relative discriminant of the extension by $\Delta_{k_{1} / k_{2}}$, which is an ideal in the ring of integers of $k_{2}$. If $k_{2}$ is either $\mathbb{Q}_{p}$ or $\mathbb{Q}$, we denote $\Delta_{k_{1} / k_{2}}$ by $\Delta_{k_{1}}$. We also denote the classical absolute discriminant of $k_{1}$ over $\mathbb{Q}$ by the same symbol $\Delta_{k_{1}}$. Since this number generates the ideal $\Delta_{k_{1}}$, the resulting notational identification is harmless.

We now return to $k$. The symbols $r_{1}, r_{2}, h_{k}, R_{k}$ and $e_{k}$ will denote respectively, the number of real places, the number of imaginary places, the class number, the regulator and the number of roots of unity contained in $k$. We put

$$
\mathfrak{C}_{k}=2^{r_{1}}(2 \pi)^{r_{2}} h_{k} R_{k} e_{k}^{-1} .
$$


We next define notations concerning adèles and idèles (see [31]). The ring of adèles, the group of idèles and the adèlic absolute value of $k$ are denoted by $\mathbb{A}, \mathbb{A}^{\times}$and || respectively. Let $\mathbb{A}^{1}=\left\{t \in \mathbb{A}^{\times}|| t \mid=1\right\}$ and $\mathbb{A}_{\mathrm{f}}$ be the finite part of $\mathbb{A}$. For $\lambda \in \mathbb{R}_{+}, \underline{\lambda} \in \mathbb{A}^{\times}$is the idèle whose component at any infinite place is $\lambda^{1 /[k: \mathbb{Q}]}$ and whose component at any finite place is 1 . Then $|\underline{\lambda}|=\lambda$.

We choose a Haar measure $d x$ on $\mathbb{A}$ so that $\int_{\mathbb{A} / k} d x=1$. For any $v \in \mathfrak{M}_{\mathrm{f}}$, we choose a Haar measure $d x_{v}$ on $k_{v}$ so that $\int_{\mathcal{O}_{v}} d x_{v}=1$. Let $d x_{v}$ be the Lebesgue measure if $v \in \mathfrak{M}_{\mathbb{R}}$, and two times the Lebesgue measure if $v \in \mathfrak{M}_{\mathbb{C}}$. It is known that $d x=\left|\Delta_{k}\right|^{-1 / 2} \prod_{v} d x_{v}$ (see [31, p. 91]).

We define a Haar measure $d^{\times} t^{1}$ on $\mathbb{A}^{1}$ so that $\int_{\mathbb{A}^{1} / k^{\times}} d^{\times} t^{1}=1$. Using this measure, we choose a Haar measure $d^{\times} t$ on $\mathbb{A}^{\times}$so that

$$
\int_{\mathbb{A}^{\times}} f(t) d^{\times} t=\int_{0}^{\infty} \int_{\mathbb{A}^{1}} f\left(\underline{\lambda} t^{1}\right) d^{\times} \lambda d^{\times} t^{1},
$$

where $d^{\times} \lambda=\lambda^{-1} d \lambda$. For any $v \in \mathfrak{M}_{\mathrm{f}}$, we choose a Haar measure $d^{\times} t_{v}$ on $k_{v}^{\times}$ so that $\int_{\mathcal{O}_{v}^{\times}} d^{\times} t_{v}=1$. Let $d^{\times} t_{v}=\left|t_{v}\right|_{v}^{-1} d t_{v}$ if $v \in \mathfrak{M}_{\infty}$.

We later have to consider the product of local measures, and for that purpose it is convenient to denote the product of local measures on $\mathbb{A}, \mathbb{A}^{\times}$as follows

$$
d_{\mathrm{pr}} x=\prod_{v} d x_{v}, d_{\mathrm{pr}}^{\times} t=\prod_{v} d^{\times} t_{v}
$$

It is well-known (see [31, pp. 91, 95]) that

$$
d x=\left|\Delta_{k}\right|^{-1 / 2} d_{\mathrm{pr}} x, d^{\times} t=\mathfrak{C}_{k}^{-1} d_{\mathrm{pr}}^{\times} t .
$$

Let $\zeta_{k}(s)$ be the Dedekind zeta function of $k$. We define

$$
Z_{k}(s)=\left|\Delta_{k}\right|^{\frac{s}{2}}\left(\pi^{-\frac{s}{2}} \Gamma\left(\frac{s}{2}\right)\right)^{r_{1}}\left((2 \pi)^{1-s} \Gamma(s)\right)^{r_{2}} \zeta_{k}(s) .
$$

This definition differs from that in $\left[31\right.$, p. 129] by the inclusion of the $\left|\Delta_{k}\right|^{s / 2}$ factor and from that in [35] by a factor of $(2 \pi)^{r_{2}}$. It is known $([31$, p. 129]) that

$$
\operatorname{Res}_{s=1} \zeta_{k}(s)=\left|\Delta_{k}\right|^{-\frac{1}{2}} \mathfrak{C}_{k}, \quad \text { and so } \quad \operatorname{Res}_{s=1} Z_{k}(s)=\mathfrak{C}_{k}
$$

For positive integers $l, m$, we define $\mathrm{M}(l, m)$ to be the set of $l \times m$ matrices. We denote the zero matrix of $\mathrm{M}(l, m)$ by $0_{l, m}$. If there is no confusion, we may write 0 instead of $0_{l, m}$. We denote the unit matrix of $\mathrm{M}(m, m)$ by $I_{m}$. 


\section{$\S 3 . \quad$ Structure of the Orbit Space}

In this section, we assume that $k$ is an arbitrary field. We denote its separable closure by $k^{\mathrm{sep}}$. The main purpose of this section is to investigate the relation between the sets of $k$-forms of orthogonal groups of various types and the set of rational orbits in the space of quadratic forms.

Let $n \geq 1$ be an integer. We consider the following pair $(G, V)$ :

$$
G=\mathrm{GL}(1) \times \mathrm{GL}(n), V=\mathrm{Sym}^{2} \mathrm{Aff}^{n}
$$

where $\mathrm{Sym}^{2} \mathrm{Aff}^{n}$ is the space of $n$-ary quadratic forms over $k$. In this paper, we mainly investigate (3.1) for $n \geq 3$. We express an element $x \in V$ as

$$
x[v]=\sum_{1 \leq i \leq j \leq n} x_{i j} v_{i} v_{j}
$$

where $v=\left(v_{1}, \cdots, v_{n}\right)$ ( $v$ is an $n$-dimensional row vector) and $v_{1}, \cdots, v_{n}$ are variables.

We associate to $x$, the symmetric matrix

$$
M_{x}=\left(\begin{array}{cccc}
2 x_{11} & x_{12} & \cdots & x_{1 n} \\
x_{12} & 2 x_{22} & \ddots & \vdots \\
\vdots & \ddots & \ddots & x_{n-1 n} \\
x_{1 n} & \cdots & x_{n-1 n} & 2 x_{n n}
\end{array}\right) .
$$

If $\operatorname{ch} k \neq 2$ then $x[v]=2^{-1} v M_{x}{ }^{t} v$ and we can identify $M_{x}$ with $x$. Let $n^{\prime} \geq 1$ and $u \in \mathrm{M}\left(n^{\prime}, n\right)$. We denote $2^{-1} u M_{x}{ }^{t} u$ by $x[u]$.

We define an action of $g=\left(t_{0}, g_{1}\right) \in G=\mathrm{GL}(1) \times \mathrm{GL}(n)$ on $V$ as follows:

$$
(g x)[v]=t_{0} x\left[v g_{1}\right] .
$$

Let $\widetilde{T}=\operatorname{Ker}(G \rightarrow \operatorname{GL}(V))$ and $\widetilde{G}=G / \widetilde{T}$. It is easy to see that

$$
\widetilde{T}=\left\{\left(\widetilde{t}_{0}^{-2}, \widetilde{t}_{0} I_{n}\right) \mid \widetilde{t}_{0} \in \mathrm{GL}(1)\right\} .
$$

We put

$$
P(x)=\left\{\begin{array}{lll}
\frac{1}{2} \operatorname{det} M_{x} & n & \text { odd }, \\
\operatorname{det} M_{x} & n & \text { even. }
\end{array}\right.
$$

We define a character $\chi$ of $G$ as follows:

$$
\chi(g)=t_{0}^{n} \operatorname{det} g_{1}^{2} \quad\left(g=\left(t_{0}, g_{1}\right) \in G\right) .
$$


Then $P(g x)=\chi(g) P(x)$. We say that a point $x \in V$ is semi-stable if $P(x) \neq 0$. We denote the set of semi-stable points of $V$ by $V^{\text {ss }}$.

For the rest of this paper, we put

$$
H=\left(\begin{array}{ll}
0 & 1 \\
1 & 0
\end{array}\right) .
$$

Let $w, w^{\prime}$ be the elements of $V$ such that

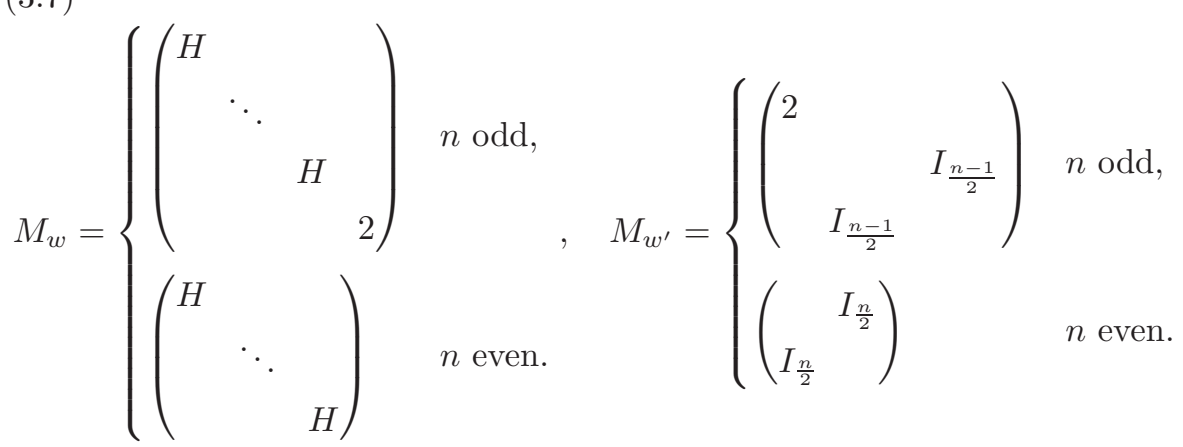

It is easy to see that $P(w)= \pm 1$ and so $w \in V_{k}^{\text {ss }}$. It is obvious that there exists a permutation matrix $\sigma$ such that $\sigma w=w^{\prime}$, which implies that $G_{k} w=G_{k} w^{\prime}$. So $P\left(w^{\prime}\right)= \pm 1$ also. If $n=2 r$ is even, we can choose such $\sigma$ so that the $(j, 2 j-1)$-entry and the $(r+j, 2 j)$-entry are 1 for $1 \leq j \leq r$. The point $w$ is more convenient for our purposes, but many textbooks on Lie groups use $w^{\prime}$ to describe the split orthogonal groups.

If $x \in V^{\text {ss }}$ then we write

$$
G_{x}=\{g \in G \mid g x=x\}, \quad \widetilde{G}_{x}=\{g \in \widetilde{G} \mid g x=x\} .
$$

We regard GL( $n)$ as a subgroup of $G$ by the natural map GL $(n) \ni g \mapsto(1, g) \in$ $G$. We define subgroups $\mathrm{GO}(x), \mathrm{O}(x)$ and $\mathrm{SO}(x)$ of $\mathrm{GL}(n)$ respectively as follows:

$$
\begin{aligned}
\mathrm{GO}(x) & =\{g \in \mathrm{GL}(n) \mid \exists \gamma(g) \in \mathrm{GL}(1) \text { s.t. } g x[v]=\gamma(g) x[v]\}, \\
\mathrm{O}(x) & =\{g \in \mathrm{GL}(n) \mid g x[v]=x[v]\}, \\
\mathrm{SO}(x) & =\mathrm{O}(x) \cap \mathrm{SL}(n) .
\end{aligned}
$$

We denote the identity component of $\mathrm{GO}(x)$ by $\mathrm{GO}(x)^{\circ}$. We call the map

$$
\gamma: \mathrm{GO}(x) \ni g \mapsto \gamma(g) \in \mathrm{GL}(1)
$$

in the definition of $\mathrm{GO}(x)$, the multiplicator of $\mathrm{GO}(x)$. 
By simple Lie algebra computations, one can show that the groups $\mathrm{GO}(x)$ and $\mathrm{SO}(x)$ are smooth algebraic groups over any $k$ (even if $\operatorname{ch} k=2$ ). The group $\mathrm{O}(x)$ is a smooth algebraic group over $k$ if $\operatorname{ch} k \neq 2$. We consider the above groups only set-theoretically if $\operatorname{ch} k=2$. For the rest of this section, we assume that $\operatorname{ch} k \neq 2$. It is well-known that $\mathrm{SO}(x)$ is the identity component of $\mathrm{O}(x)$. It is reductive if $n \geq 2$ and semi-simple if $n \geq 3$.

Let $Z=\left\{t I_{n} \mid t \in \operatorname{GL}(1)\right\}$. Then $Z$ is the center of $\operatorname{GO}(x)$. If $n$ is odd (resp. even) then $Z \cap \mathrm{SO}(x)=\left\{I_{n}\right\}$ (resp. $Z \cap \mathrm{SO}(x)=\left\{ \pm I_{n}\right\}$ ). In both cases $Z \cap \mathrm{SO}(x)$ is the center of $\mathrm{SO}(x)$. We define

$$
\mathrm{PSO}(x)=\mathrm{SO}(x) /(Z \cap \mathrm{SO}(x)), \quad \mathrm{PGO}(x)=\mathrm{GO}(x) / Z .
$$

It is easy to see that $\operatorname{GO}(x)^{\circ} / Z$ is the identity component of $\operatorname{PGO}(x)$. It is well-known that $\mathrm{PSO}(x) \cong \operatorname{PGO}(x)^{\circ}$ as algebraic groups (however, if $n$ is even then the set-theoretic quotients $\mathrm{SO}(x)_{k} /\left\{ \pm I_{n}\right\}, \mathrm{GO}(x)_{k}^{\circ} / Z_{k}$ may not coincide).

The following lemma is easy to prove and we simply state it without proof.

Lemma 3.9. If $x \in V^{\mathrm{ss}}$ then the projection to the second factor induces an isomorphism $G_{x} \cong \mathrm{GO}(x)$.

Let $n \geq 3$ for the rest of this section. Let Aut $(\mathrm{SO}(w))$ and $\operatorname{Aut}\left(\mathrm{PGO}(w)^{\circ}\right)$ (resp. $\operatorname{Int}(\mathrm{SO}(w))$ and $\operatorname{Int}\left(\mathrm{PGO}(w)^{\circ}\right)$ ) be the automorphism groups (resp. the inner automorphism groups) of $\mathrm{SO}(w)$ and $\mathrm{PGO}(w)^{\circ}$.

If $h \in \mathrm{PGO}(w)$ then we define an automorphism $\operatorname{Ad}(h)$ of $\mathrm{PGO}(w)^{\circ}$ as follows:

$$
\operatorname{Ad}(h): \operatorname{PGO}(w)^{\circ} \ni x \mapsto h x h^{-1} \in \operatorname{PGO}(w)^{\circ} .
$$

The group $\operatorname{PGO}(w)^{\circ}$ is semi-simple since $n \geq 3$. If we denote the Dynkin diagram of $\operatorname{PGO}(w)^{\circ}$ by $\operatorname{Dyn}\left(\mathrm{PGO}(w)^{\circ}\right)$, then applying Proposition [1, p. 190] to $\operatorname{PGO}(w)^{\circ}$, there exists a natural injection

$$
\operatorname{Aut}\left(\operatorname{PGO}(w)^{\circ}\right) / \operatorname{Int}\left(\operatorname{PGO}(w)^{\circ}\right) \rightarrow \operatorname{Aut}\left(\operatorname{Dyn}\left(\operatorname{PGO}(w)^{\circ}\right)\right) \text {. }
$$

Since the Dynkin diagrams of $\mathrm{PGO}(w)^{\circ}$ and $\mathrm{SO}(w)$ are of the same type,

$$
\operatorname{Aut}\left(\operatorname{Dyn}\left(\operatorname{PGO}(w)^{\circ}\right)\right) \cong \begin{cases}\{1\} & n \text { is odd, } \\ \mathbb{Z} / 2 \mathbb{Z} & n \neq 8 \text { is even, } \\ \mathfrak{S}_{3} & n=8\end{cases}
$$

where $\mathfrak{S}_{3}$ is the symmetric group of degree 3.

Lemma 3.11. If $n \geq 3$ then

$$
\operatorname{PGO}(w) \cong \operatorname{Aut}\left(\operatorname{PGO}(w)^{\circ}\right) \cong \operatorname{Aut}(\mathrm{SO}(w)) .
$$


Proof. We first assume that $n \geq 3$ is odd. We briefly review the proof of the fact that $\mathrm{GO}(w)^{\circ}=\mathrm{GO}(w)$ (which implies that $\mathrm{PGO}(w)^{\circ}=\operatorname{PGO}(w)$ ). It is well-known that $\mathrm{SO}(w)$ is connected as an algebraic group. Since $Z \cong$ $\mathrm{GL}(1) \subset \mathrm{GO}(w)$ is connected, $Z \subseteq \mathrm{GO}(w)^{\circ}$. We show that $\mathrm{GO}(w)_{\bar{k}}=$ $\mathrm{SO}(w)_{\bar{k}} Z_{\bar{k}}$, which proves that $\mathrm{GO}(w)$ is connected. It is easy to see that if $g \in \operatorname{GO}(w)_{\bar{k}}$ then there exists $t \in Z_{\bar{k}}$ such that $\gamma(t)=\gamma(g)$. So we may assume that $g \in \mathrm{O}(w)$. Then $\operatorname{det} g= \pm 1$. Since $-I_{n} \in Z$ and $\operatorname{det}\left(-I_{n}\right)=-1$, $g \in \mathrm{SO}(w)_{\bar{k}} Z_{\bar{k}}$. This proves that $\mathrm{GO}(w)$ is connected.

The map $\operatorname{PGO}(w) \ni h \mapsto \operatorname{Ad}(h) \in \operatorname{Aut}(\operatorname{PGO}(w))$ is surjective by (3.10). Moreover this map is injective because the center of $\operatorname{PGO}(w)$ is trivial. Therefore, $\mathrm{PGO}(w)$ and Aut $(\mathrm{PGO}(w))$ are isomorphic by the map $\mathrm{PGO}(w) \ni h \mapsto$ $\operatorname{Ad}(h) \in \operatorname{Aut}(\operatorname{PGO}(w))$. The argument is similar for Aut $(\mathrm{SO}(w))$.

We next assume that $n$ is even. For $g \in \operatorname{PGO}(x)$, we define $\operatorname{Ad}(g) \in$ Aut $\left(\operatorname{PGO}(x)^{\circ}\right)$ similarly as above. We first prove that

$$
\operatorname{Aut}\left(\operatorname{PGO}(w)^{\circ}\right) / \operatorname{Int}\left(\operatorname{PGO}(w)^{\circ}\right) \cong \mathbb{Z} / 2 \mathbb{Z} \text {. }
$$

(It is proved in $[23$, p. 90$]$ that $\operatorname{Aut}(\mathrm{SO}(w)) / \operatorname{Int}(\mathrm{SO}(w)) \cong \mathbb{Z} / 2 \mathbb{Z})$. We put

$$
\tau=\left(\begin{array}{cc}
I_{n-2} & \\
& H
\end{array}\right), \quad \tau^{\prime}=\left(\begin{array}{ccc}
I_{\frac{n}{2}-1} & & \\
& & 1 \\
& I_{\frac{n}{2}-1}
\end{array}\right) .
$$

If $\sigma$ is the permutation matrix defined after (3.7) then simple computations show that $\sigma \tau \sigma^{-1}=\tau^{\prime}$. It is easy to see that $\tau \in \mathrm{O}(w)$ and $\tau^{\prime} \in \mathrm{O}\left(w^{\prime}\right)$.

It is easy to see that $\operatorname{Ad}\left(\tau^{\prime}\right)$ stabilizes the standard Borel subgroup of $\operatorname{PGO}\left(w^{\prime}\right)^{\circ}$ and exchanges the last two roots of the Dynkin diagram of the group $\operatorname{PGO}\left(w^{\prime}\right)^{\circ}$. So $\operatorname{Ad}\left(\tau^{\prime}\right)$ is an outer automorphism of $\operatorname{PGO}\left(w^{\prime}\right)^{\circ}$. This implies that $\operatorname{Ad}(\tau)$ is an outer automorphism of $\operatorname{PGO}(w)^{\circ}$ also. Thus, by (3.10),

$$
\text { Aut }\left(\operatorname{PGO}(w)^{\circ}\right) / \operatorname{Int}\left(\operatorname{PGO}(w)^{\circ}\right) \cong \mathbb{Z} / 2 \mathbb{Z}
$$

for $n \neq 8$.

Suppose that $n=8$. We assume that $\operatorname{Aut}\left(\operatorname{PGO}(w)^{\circ}\right) / \operatorname{Int}\left(\operatorname{PGO}(w)^{\circ}\right) \cong$ $\mathfrak{S}_{3}$ and deduce a contradiction.

We denote the spin group of degree 8 by $\operatorname{Spin}(8)$. Then

$$
\operatorname{Aut}(\operatorname{Spin}(8)) / \operatorname{Int}(\operatorname{Spin}(8)) \cong \mathfrak{S}_{3} \text {. }
$$

So every automorphism of $\operatorname{Spin}(8)$ is realized by an element of $\operatorname{Aut}\left(\operatorname{PGO}(w)^{\circ}\right)$. Let $(\rho, W)$ be the vector representation of $\operatorname{Spin}(8)$. By assumption, there exists 
$\phi \in \operatorname{Aut}\left(\operatorname{PGO}(w)^{\circ}\right)$ such that $\rho \circ \phi$ is one of the half-spin representations. Note that $\rho \circ \phi(-1)=\chi(-1)$ is the identity. Here -1 is the scalar -1 in the Clifford algebra. However, the image of $-1 \in \operatorname{Spin}(8)$ by the half-spin representation is non-trivial, which is a contradiction. Therefore, (3.12) holds for $n=8$ also.

Since $h$ maps to the non-trivial element of

$$
\text { Aut }\left(\operatorname{PGO}(w)^{\circ}\right) / \operatorname{Int}\left(\operatorname{PGO}(w)^{\circ}\right) \cong \mathbb{Z} / 2 \mathbb{Z}
$$

and the center of $\operatorname{PGO}(w)$ is trivial, $\operatorname{PGO}(w) \cong \operatorname{Aut}\left(\operatorname{PGO}(w)^{\circ}\right)$. The rest of the argument (including that for Aut $(\mathrm{SO}(w))$ ) is similar to the case where $n$ is odd.

Lemma 3.11 implies that $\left[\mathrm{GO}(w): \mathrm{GO}(w)^{\circ}\right]=2$ if $n$ is even.

Lemma 3.14. If $n \geqq 3$ is odd then $G_{w} \cong \mathrm{SO}(w) \times \widetilde{T}$.

Proof. Let $\left(t_{0}, g\right) \in G_{w \bar{k}}$. All automorphisms of $\mathrm{SO}(w)$ are inner by Lemma 3.11. So there exists $\bar{g} \in \mathrm{SO}(w)_{\bar{k}}$ such that $\operatorname{Ad}(g)(h)=g h g^{-1}=\bar{g} h \bar{g}^{-1}$ for all $h \in \mathrm{SO}(w)_{\bar{k}}$. Since $\bar{g}^{-1} g$ commutes with all elements of $\mathrm{SO}(w)_{\bar{k}}$, there exists $t_{0}^{\prime} \in \bar{k}^{\times}$such that $g=t_{0}^{\prime} \bar{g}$. So $\mathrm{GO}(w)_{\bar{k}}=\mathrm{SO}(w)_{\bar{k}} \widetilde{T}_{\bar{k}}$. Since $\mathrm{SO}(w)_{\bar{k}} \cap \widetilde{T}_{\bar{k}}=$ $\left\{\left(1, I_{n}\right)\right\}$, the map

$$
\mathrm{SO}(w)_{\bar{k}} \times \widetilde{T}_{\bar{k}} \rightarrow G_{w \bar{k}}
$$

is an isomorphism.

Simple Lie algebra computations show that the differential of the above map is an isomorphism. Note that $\mathrm{SO}(w) \times \widetilde{T}$ and $G_{w}$ are both smooth over $k$ and there is a natural map $\phi_{w}: \mathrm{SO}(w) \times \widetilde{T} \rightarrow G_{w}$. Since $\phi_{w}$ is an isomorphism over $\bar{k}$, it is an isomorphism over $k$.

We next consider the relation between the sets of $k$-forms of the groups $\mathrm{SO}(w), \operatorname{PGO}(w)^{\circ}$ and the orbit space $G_{k} \backslash V_{k}^{\text {ss }}$. Let $G_{1}$ and $G_{2}$ be algebraic groups over $k$. We say that $G_{2}$ is a $k$-form of $G_{1}$ if there exists a separable algebraic extension $K / k$ such that $G_{1} \times_{k} K \cong G_{2} \times_{k} K$. We define the first Galois cohomology set $\mathrm{H}^{1}(k, G)$ for an algebraic group $G$ over $k$ in the same manner as in [13, p. 317], i.e. a 1-cocycle $h=\left\{h_{\eta}\right\}_{\eta \in \operatorname{Gal}\left(k^{\text {sep }} / k\right)}$ satisfies the condition $h_{\eta_{1} \eta_{2}}=h_{\eta_{2}} h_{\eta_{1}}^{\eta_{2}}$ for all $\eta_{1}, \eta_{2} \in \operatorname{Gal}\left(k^{\mathrm{sep}} / k\right)$.

Proposition 3.15. $\quad$ Let $n \geq 3$. The orbit space $G_{k} \backslash V_{k}^{\text {ss }}$ is in bijective correspondence with the set of $k$-isomorphism classes of algebraic groups in the form $\mathrm{SO}(x)$ where $x \in V_{k}^{\mathrm{ss}}$. It is also in bijective correspondence with the set of $k$-isomorphism classes of algebraic groups in the form $\mathrm{PGO}(x)^{\circ}$. Moreover, if $n$ is odd then the set $\{\mathrm{SO}(x)\}_{x \in G_{k} \backslash V_{k}^{\mathrm{ss}}}=\left\{\mathrm{PGO}(x)^{\circ}\right\}_{x \in G_{k} \backslash V_{k}^{\mathrm{ss}}}$ exhausts all $k$-forms of $\mathrm{SO}(w)=\mathrm{PGO}(w)^{\circ}$. 
Proof. We first assume that $n$ is odd. Using Theorem (1.7) [13, p. 318], there is a bijective map from $G_{k} \backslash V_{k}^{\text {ss }}$ to $\mathrm{H}^{1}\left(k, G_{w}\right)=\mathrm{H}^{1}(k$, Aut $(\mathrm{SO}(w))) \times$ $\mathrm{H}^{1}(k, \widetilde{T})$. Note that $\mathrm{H}^{1}(k, \widetilde{T})=\{1\}$ by Hilbert's Theorem 90. It is known that $\mathrm{H}^{1}(k$, Aut $(\mathrm{SO}(w)))$ is in bijective correspondence with the set of $k$-forms of $\mathrm{SO}(w)$ (see [23, p. 67]). Therefore, $G_{k} \backslash V_{k}^{\text {ss }}$ is in bijective correspondence with the set of $k$-forms of $\mathrm{SO}(w)$ if $n$ is odd.

We now prove that $x \in V_{k}^{\text {ss }}$ corresponds to the $k$-form $\mathrm{SO}(x)$ by this correspondence. If $x=g_{x} w$ for $g_{x}=\left(t_{x, 0}, g_{x, 1}\right) \in G_{k^{\text {sep }}}$, then $\operatorname{Ad}\left(g_{x, 1}^{-1} g_{x, 1}^{\eta}\right) \in$ Aut $\left(\mathrm{SO}(w)_{k^{\text {sep }}}\right)$. So $x$ corresponds to the class of $\left\{\operatorname{Ad}\left(g_{x, 1}^{-1} g_{x, 1}^{\eta}\right)\right\}_{\eta \in \operatorname{Gal}\left(k^{\operatorname{sep}} / k\right)}$. Let $G(x)$ be the $k$-form of $\mathrm{SO}(w)$ corresponding to $\left\{\operatorname{Ad}\left(g_{x, 1}^{-1} g_{x, 1}^{\eta}\right)\right\}_{\eta \in \operatorname{Gal}\left(k^{\text {sep }} / k\right)}$.

We show that there is a natural isomorphism $G(x)_{R} \cong \mathrm{SO}(x)_{R}$ for any $k$-algebra $R$. Let $R_{s}=R \otimes k^{\mathrm{sep}}$. We define an action of $\eta \in \operatorname{Gal}\left(k^{\mathrm{sep}} / k\right)$ on $R_{s}$ by $(r \otimes x)^{\eta}=r \otimes x^{\eta}$. Let

$$
\nu_{x}(\eta): \mathrm{SO}(w)_{R_{s}} \ni g \mapsto \operatorname{Ad}\left(g_{x, 1}^{-1} g_{x, 1}^{\eta}\right)\left(g^{\eta}\right) \in \mathrm{SO}(w)_{R_{s}} .
$$

Then the set $G(x)_{R}$ of $R$-rational points of $G(x)$ can be expressed as

$$
G(x)_{R}=\left\{g \in \mathrm{SO}(w)_{R_{s}} \mid \nu_{x}(\eta)(g)=g \quad \forall \eta \in \operatorname{Gal}\left(k^{\mathrm{sep}} / k\right)\right\} .
$$

If $g \in \mathrm{SO}(w)_{R_{s}}$ satisfies $\nu_{x}(\eta)(g)=g$ for all $\eta$, then $g_{x, 1} g g_{x, 1}^{-1} \in \operatorname{SO}(x)_{R_{s}}$ and

$$
\left(g_{x, 1} g g_{x, 1}^{-1}\right)^{\eta}=g_{x, 1} g g_{x, 1}^{-1} .
$$

So there is a natural isomorphism

$$
\mathrm{SO}(w)_{R_{s}} \supset G(x)_{R} \ni g \mapsto g_{x, 1} g g_{x, 1}^{-1} \in \mathrm{SO}(x)_{R} .
$$

Since there is a natural isomorphism $G(x)_{R} \cong \mathrm{SO}(x)_{R}$ for any $k$-algebra $R$, there is an isomorphism between the algebraic groups $G(x)$ and $\mathrm{SO}(x)$ over $k$ (see THEOREM $[20, \mathrm{p} .17]$ ). Thus, $x \in V_{k}^{\text {ss }}$ corresponds to the $k$-isomorphism class of the $k$-form $\mathrm{SO}(x)$.

We next assume that $n$ is even. We consider $\widetilde{G}_{k} \backslash V_{k}^{\text {ss }}$ instead of $G_{k} \backslash V_{k}^{\text {ss }}$. Note that $\widetilde{G}_{k} \cong G_{k} / \widetilde{T}_{k}$ since $\mathrm{H}^{1}(k, \widetilde{T})=\{1\}$. By Theorem (1.6) [13, p. 318], there is a bijective map

$$
\widetilde{G}_{k} \backslash V_{k}^{\mathrm{ss}}=G_{k} \backslash V_{k}^{\mathrm{ss}} \rightarrow \operatorname{ker}\left(\mathrm{H}^{1}\left(k, \widetilde{G}_{w}\right) \rightarrow \mathrm{H}^{1}(k, \widetilde{G})\right) .
$$

By Lemma 3.9 and Lemma 3.11, there is a bijective correspondence between

$$
\mathrm{H}^{1}\left(k, \widetilde{G}_{w}\right)=\mathrm{H}^{1}(k, \operatorname{Aut}(\mathrm{SO}(w)))
$$


and the set of $k$-forms of $\mathrm{SO}(w)$. Using (3.16) and this correspondence, we obtain a map from $G_{k} \backslash V_{k}^{\text {ss }}$ into the set of $k$-forms of $\mathrm{SO}(w)$. By the above argument, this map is injective. It can be verified that it associates $x \in V_{k}^{\text {ss }}$ to the $k$-form $\mathrm{SO}(x)$ by the same argument as in the case where $n$ is odd. The argument is similar for $k$-forms of $\operatorname{PGO}(w)^{\circ}$.

This completes the proof of the proposition.

If $n$ is even, we define a real subgroup $\mathrm{SO}^{*}(n)$ of $\mathrm{SO}(n)_{\mathbb{C}}$ as follows:

$$
\mathrm{SO}^{*}(n)=\left\{g \in \mathrm{SO}(n)_{\mathbb{C}} \mid g J g^{*}=J\right\}
$$

where $J=\left(I_{n / 2} I_{n / 2}\right)$ and $g^{*}$ is the complex conjugate of ${ }^{t} g$. It is known that $\mathrm{SO}^{*}(n)$ corresponds to the Satake diagram of type DIII and $\mathrm{SO}(x)_{\mathbb{R}}$ corresponds to the Satake diagram of type DI or DII for any $x \in V_{\mathbb{R}}^{\mathrm{ss}}$. Therefore, $\mathrm{SO}^{*}(n)$ is isomorphic to $\mathrm{SO}(w)$ over $\mathbb{C}$, but not isomorphic to $\mathrm{SO}(x)$ over $\mathbb{R}$ for any $x \in V_{\mathbb{R}}^{\text {ss }}$ (see [10, pp. 445-446, 453, 527,533]). Therefore, the $\mathbb{R}$-form $\mathrm{SO}^{*}(n)$ of $\mathrm{SO}(w)$ does not come from $G_{\mathbb{R}} \backslash V_{\mathbb{R}}^{\mathrm{ss}}$.

\section{$\S 4$. A Set of Representatives for the Local Orbit Space}

For the rest of this paper, we assume that $k$ is a number field. The main purpose of this section is to choose a set of representatives for $G_{k_{v}} \backslash V_{k_{v}}^{\text {ss }}$ for $n \geq 2$.

We assume that $v \in \mathfrak{M}_{\mathrm{f}}$. Let

$$
m_{v}=\operatorname{ord}_{v} 2, \quad \lambda_{v}=\sharp\left(k_{v}^{\times} /\left(k_{v}^{\times}\right)^{2}\right)-2 .
$$

First we review some facts concerning quadratic extensions of $k_{v}$. There is a unique unramified quadratic extension $F_{0}$ of $k_{v}$ and it is generated by a root of an irreducible polynomial

$$
p_{0}(z)=z^{2}+a_{0} z+b_{0}
$$

for a suitable choice of $a_{0}, b_{0} \in \mathcal{O}_{v}^{\times}$whose discriminant $a_{0}^{2}-4 b_{0}$ is a unit. Moreover, $F_{0}$ is also generated by the square root of a non-square unit in the form

$$
\mu_{v}=1+4 c
$$

for some $c \in \mathcal{O}_{v}^{\times}$. This $\mu_{v}$ corresponds to $\Delta$ in [22, p.164]. 
Now we consider ramified quadratic extensions of $k_{v}$. Every ramified quadratic extension $F$ of $k_{v}$ is generated by either root of an Eisenstein polynomial

$$
p(z)=z^{2}+a z+b .
$$

Let $\pi_{F}$ be a root of $p(z)$. Then $\pi_{F}$ is a uniformizer of $F$. We have $\mathcal{O}_{F}=\mathcal{O}_{v}\left[\pi_{F}\right]$ and $\Delta_{F / k_{v}}=\left(a^{2}-4 b\right) \mathcal{O}_{v}$. Let $l=\operatorname{ord}_{v}(a)$. If $l \geq m_{v}+1$, we may assume that $a=0$ by the transformation $z \mapsto z-(a / 2)$. In this case, $F$ is generated by the square root of a uniformizer of $k_{v}$ and $\Delta_{F / k_{v}}=\mathfrak{p}_{v}^{2 m_{v}+1}$. If $1 \leq l \leq m_{v}$ then $\Delta_{F / k_{v}}=\mathfrak{p}_{v}^{2 l}$ and $F$ is generated by the square root of $a^{2}-4 b$ and also by the square root of $1-4 a^{-2} b=1+\pi_{v}^{2\left(m_{v}-l\right)+1} c$ for suitable $c \in \mathcal{O}_{v}^{\times}$. This exhausts all quadratic extensions of $k_{v}$. There are $\lambda_{v}$ isomorphism classes of ramified extensions of $k_{v}$. We denote their representatives by $F_{1}, \cdots, F_{\lambda_{v}}$. Note that $F_{0}$ is the unramified extension of $k_{v}$ which corresponds to the Artin-Schreier polynomial (4.2). For each $1 \leq j \leq \lambda_{v}$, let

$$
p_{j}(z)=z^{2}+a_{j} z+b_{j}
$$

be an Eisenstein polynomial which corresponds to $F_{j}$.

It is known that the orbit space $G_{k} \backslash V_{k}^{\text {ss }}$ for $n=2$ is in bijective correspondence with the set of isomorphism classes of Galois extensions of $k$ which are splitting fields of degree two equations without multiple roots (see [33, pp. 285, 309-310]).

Let

$$
A_{v, \text { in }}=\left(\begin{array}{cc}
2 & a_{0} \\
a_{0} & 2 b_{0}
\end{array}\right), \quad A_{v,(\mathrm{rm}, j)}=\left(\begin{array}{cc}
2 & a_{j} \\
a_{j} & 2 b_{j}
\end{array}\right) 1 \leq j \leq \lambda_{v}
$$

Then, for $n=2$, we can choose a set of representatives for $G_{k_{v}} \backslash V_{k_{v}}^{\text {ss }}$ as follows:

$$
\left\{H=\left(\begin{array}{ll}
0 & 1 \\
1 & 0
\end{array}\right), A_{v, \mathrm{in}}, A_{v,(\mathrm{rm}, 1)}, \cdots, A_{v,\left(\mathrm{rm}, \lambda_{v}\right)}\right\} \subset \mathrm{M}(2,2)_{k_{v}} .
$$

Now we consider all places $v \in \mathfrak{M}$ again. We recall definitions of some invariants of quadratic forms over $k_{v}$. An $n$-ary quadratic form $x$ is called isotropic if there exists a nonzero vector $v \in k_{v}^{n}$ such that $x[v]=0$, anisotropic if not. It is known that by the action of $\mathrm{GL}(n)_{k_{v}}, x \in V_{k_{v}}^{\mathrm{ss}}$ can be made into the following form:

$$
\left(\begin{array}{llll}
H & & & \\
& \ddots & & \\
& & H & \\
& & & x^{\prime}
\end{array}\right)
$$


where $x^{\prime}$ is an anisotropic quadratic form. If the size of $x^{\prime}$ is $m_{0} \times m_{0}$ then $m_{0}$ does not depend on the choice of $x^{\prime}$ (see [22, pp. 98-99]). We call $\left(n-m_{0}\right) / 2$ the Witt index of $x$. It is the split rank of $\mathrm{SO}(x)$. It is easy to see that $\alpha x^{\prime}$ is anisotropic for all $\alpha \in \mathrm{GL}(1)_{k_{v}}$ if $x^{\prime}$ is anisotropic. Since $\alpha H \in \mathrm{GL}(2)_{k_{v}} H$ for all $\alpha \in k_{v}^{\times}$, the Witt index is also invariant under the action of $G_{k_{v}}$. If $v \in \mathfrak{M}_{\mathbb{C}}$ then the Witt index is clearly $[n / 2]$. If $v \in \mathfrak{M}_{\mathbb{R}}$ and the Witt index of $x \in V_{\mathbb{R}}^{\text {ss }}$ is $m$, then

$$
\left(\begin{array}{cc}
-I_{m} & \\
& I_{n-m}
\end{array}\right) \text { or }\left(\begin{array}{cc}
I_{m} & \\
& -I_{n-m}
\end{array}\right)
$$

belongs to $\operatorname{GL}(n)_{\mathbb{R}} x$. So the signature of $x$ is $(n-m, m)$ or $(m, n-m)$.

Let $x \in V_{k_{v}}^{\text {ss }}$. We define $d_{v}(x)$ to be the class of $\operatorname{det} M_{x}$ in $k_{v}^{\times} /\left(k_{v}^{\times}\right)^{2}$, i.e.,

$$
d_{v}(x) \equiv \operatorname{det} M_{x} \equiv\left\{\begin{array}{lll}
2 P(x) & n \text { odd } \\
P(x) & n \text { even }
\end{array} \quad \bmod \left(k_{v}^{\times}\right)^{2} .\right.
$$

Note that this congruence is multiplicative. We always regard $d_{v}(x)$ as an element of $k_{v}^{\times} /\left(k_{v}^{\times}\right)^{2}$. It is clear that $d_{v}$ is invariant under the action of $\operatorname{GL}(n)_{k_{v}}$. In this paper, we call $d_{v}(x)$ the classical discriminant of $x$.

We next define the Hasse symbol of $x \in V_{k_{v}}^{\text {ss }}$. It is known that there exists $a \in \operatorname{GL}(n)_{k_{v}} x$ such that

$$
M_{a}=\left(\begin{array}{ccc}
\alpha_{1} & & 0 \\
& \ddots & \\
0 & & \alpha_{n}
\end{array}\right)\left(\alpha_{1}, \cdots, \alpha_{n} \in k_{v}^{\times}\right)
$$

(see [22, p. 90]). We define the Hasse symbol $S_{v}(x)$ by

$$
S_{v}(x)=\prod_{1 \leq i \leq j \leq n}\left(\alpha_{i}, \alpha_{j}\right)_{v}
$$

where $(,)_{v}$ is the Hilbert symbol. The Hasse symbol $S_{v}(x)$ does not depend on the choice of $a$ and is invariant under the action of $\operatorname{GL}(n)_{k_{v}}$ (see [22, p. 167]).

In the classical theory of quadratic forms, a quadratic form

$$
x(v)=\sum_{1 \leq i \leq j \leq n} x_{i j} v_{i} v_{j}
$$

corresponds to the symmetric matrix $2^{-1} M_{x}$. The symbols $d_{v}(x)$ and $S_{v}(x)$ are the discriminant and the Hasse symbol of $2 M_{x}$ in [22, pp. 87, 167]. It can be verified that the above $d_{v}(x)$ and $S_{v}(x)$ have the same properties as the discriminant and the Hasse symbol in [22, pp. 87, 167].

The following theorem is Theorem (63:20) [22, p. 170]. 
Theorem 4.6. Suppose that $x, y \in V_{k_{v}}^{\mathrm{ss}}$. Then $\mathrm{GL}(n)_{k_{v}} x=\mathrm{GL}(n)_{k_{v}} y$ if and only if $d_{v}(x)=d_{v}(y)$ and $S_{v}(x)=S_{v}(y)$.

We define the modified Hasse symbol $\widetilde{S}_{v}$ of $x \in V_{k_{v}}^{\text {ss }}$ by

$$
\widetilde{S}_{v}(x)=S_{v}\left(\operatorname{det} M_{x} x\right)
$$

if $n \geq 3$ is odd.

Lemma 4.7. Suppose that $n \geq 3$ is odd, $v \in \mathfrak{M}_{\mathrm{f}}$ and $x, y \in V_{k_{v}}^{\mathrm{ss}}$. Then $G_{k_{v}} x=G_{k_{v}} y$ if and only if $\widetilde{S}_{v}(x)=\widetilde{S}_{v}(y)$.

Proof. Since $n$ is odd,

$d_{v}\left(\left(\operatorname{det} M_{x}\right) x\right) \equiv \operatorname{det}\left(\left(\operatorname{det} M_{x}\right) M_{x}\right)=\left(\operatorname{det} M_{x}\right)^{n+1} \equiv 1 \bmod \left(k_{v}^{\times}\right)^{2}$.

Let $\left(t_{0}, g_{1}\right) \in G_{k_{v}}$ and $y=\left(t_{0}, g_{1}\right) x$. Then

$$
\left(\operatorname{det} M_{y}\right) y=\left(t_{0}^{n+1} \operatorname{det} g_{1}^{2}, g_{1}\right)\left(\operatorname{det} M_{x}\right) x \text {. }
$$

Since $\left(\operatorname{det} M_{y}\right) y$ can be regarded as $\left(1,\left(t_{0}^{(n+1) / 2} \operatorname{det} g_{1}\right) g_{1}\right)\left(\operatorname{det} M_{x}\right) x$ and $S_{v}$ is invariant under the action of $\operatorname{GL}(n)_{\tilde{S}_{v}}, S_{v}\left(\left(\widetilde{S}_{v} M_{y}\right) y\right)=S_{v}\left(\left(\operatorname{det} M_{x}\right) x\right)$.

Conversely, let $x, y \in V_{k_{v}}^{\text {ss }}$ and $\widetilde{S}_{v}(x)=\widetilde{S}_{v}(y)$. Since

$$
d_{v}\left(\left(\operatorname{det} M_{x}\right) x\right) \equiv d_{v}\left(\left(\operatorname{det} M_{y}\right) y\right) \equiv 1,
$$

we have

$$
\operatorname{GL}(n)_{k_{v}}\left(\left(\operatorname{det} M_{x}\right) x\right)=\operatorname{GL}(n)_{k_{v}}\left(\left(\operatorname{det} M_{y}\right) y\right)
$$

by Theorem 4.6. Since $\operatorname{det} M_{x} \operatorname{det} M_{y}^{-1}$ is a scalar, $G_{k_{v}} x=G_{k_{v}} y$.

Suppose that $n$ is even, $x \in V_{k_{v}}^{\mathrm{ss}}$ and $\alpha \in k_{v}^{\times}$. Then

$$
\widetilde{S}_{v}(\alpha x)=S_{v}\left(\alpha^{n+1} \operatorname{det} M_{x} x\right)=S_{v}\left(\alpha \operatorname{det} M_{x} x\right) .
$$

If $y \in V_{k_{v}}^{\text {ss }}$ then

$$
S_{v}(\alpha y)=\left(\alpha,(-1)^{\frac{n(n+1)}{2}}\left(\operatorname{det} M_{y}\right)^{n+1}\right) S_{v}(y)=\left(\alpha,(-1)^{\frac{n(n+1)}{2}} \operatorname{det} M_{y}\right) S_{v}(y) .
$$

So

$$
\begin{aligned}
\widetilde{S}_{v}(\alpha x) & =\left(\alpha,(-1)^{\frac{n(n+1)}{2}}\left(\operatorname{det} M_{x}\right)^{n+1}\right) S_{v}\left(\operatorname{det} M_{x} x\right) \\
& =\left(\alpha,(-1)^{\frac{n(n+1)}{2}} \operatorname{det} M_{x}\right) S_{v}\left(\operatorname{det} M_{x} x\right)=\left(\alpha,(-1)^{\frac{n(n+1)}{2}} \operatorname{det} M_{x}\right) \widetilde{S}_{v}(x) .
\end{aligned}
$$


If $\operatorname{ord}\left(\operatorname{det} M_{x}\right)=1$ then there exists $\alpha \in k_{v}^{\times}$such that $\left(\alpha,(-1)^{\frac{n(n+1)}{2}} \operatorname{det} M_{x}\right)=$ -1 ((63:11a) [22, p. 165]). Therefore, $\widetilde{S}_{v}(x)$ is not $G_{k_{v}}$-invariant if $n$ is even.

We now choose a set of representatives for $G_{k_{v}} \backslash V_{k_{v}}^{\text {ss }}$. We first consider the case $v \in \mathfrak{M}_{\mathrm{f}}$. Suppose that $n \geq 3$ is odd. By the above lemma, there are at most two $G_{k_{v}}$-orbits in $V_{k_{v}}^{\text {ss }}$. We define quadratic forms $w_{v, \text { sp }}^{0}$ and $w_{v, \text { rm }}^{0}$ over $k_{v}$ as follows:

$$
w_{v, \mathrm{sp}}^{0}=(2), \quad w_{v, \mathrm{rm}}^{0}=\left(\begin{array}{lll}
A_{v, \mathrm{in}} & \\
& 2 \pi_{v}
\end{array}\right)
$$

where $A_{v \text {,in }}$ is the symmetric matrix in (4.5). The indices "sp" and "rm" stand respectively for "split" and "ramified". It is easy to see that the above $w_{v, \mathrm{sp}}^{0}$ and $w_{v, \mathrm{rm}}^{0}$ are both anisotropic.

Definition 4.9. Let $n \geq 3$ be odd. We define $n$-ary quadratic forms $w_{v, \mathrm{sp}}, w_{v, \mathrm{rm}} \in V_{k_{v}}^{\mathrm{ss}}$ as follows:

$$
w_{v, \mathrm{sp}}=\left(\begin{array}{cccc}
H & & & \\
& \ddots & & \\
& & H & \\
& & & w_{v, \mathrm{sp}}^{0}
\end{array}\right)=w, \quad w_{v, \mathrm{rm}}=\left(\begin{array}{cccc}
H & & & \\
& \ddots & & \\
& & H & \\
& & & w_{v, \mathrm{rm}}^{0}
\end{array}\right) \text {. }
$$

If there is no confusion, we write $w_{\mathrm{sp}}^{0}, w_{\mathrm{rm}}^{0}$ and $w_{\mathrm{sp}}, w_{\mathrm{rm}}$.

Proposition 4.11. Let $n \geq 3$ be odd. If $v \in \mathfrak{M}_{\mathrm{f}}$ then $\left\{w_{\mathrm{sp}}, w_{\mathrm{rm}}\right\}$ is a complete set of representatives for $G_{k_{v}} \backslash V_{k_{v}}^{\mathrm{ss}}$. If $v \in \mathfrak{M}_{\mathrm{f}} \backslash \mathfrak{M}_{\mathrm{dy}}$ then

$$
\widetilde{S}_{v}\left(w_{\mathrm{sp}}\right)=1, \quad \widetilde{S}_{v}\left(w_{\mathrm{rm}}\right)=-1 .
$$

Proof. The Witt indices of $w_{\mathrm{sp}}$ and $w_{\mathrm{rm}}$ are respectively $(n-1) / 2$ and $(n-3) / 2$. So $G_{k_{v}} w_{\mathrm{sp}} \neq G_{k_{v}} w_{\mathrm{rm}}$. By Lemma 4.7 , there are at most two $G_{k_{v}}-$ orbits in $V_{k_{v}}^{\mathrm{ss}}$. Therefore, $\left\{w_{\mathrm{sp}}, w_{\mathrm{rm}}\right\}$ is a complete set of representatives for $G_{k_{v}} \backslash V_{k_{v}}^{\text {ss }}$.

Suppose that $v \in \mathfrak{M}_{\mathrm{f}} \backslash \mathfrak{M}_{\mathrm{dy}}$. It can easily be verified that $\left(\alpha_{1}, \alpha_{2}\right)=1$ for all $\alpha_{1}, \alpha_{2} \in \mathcal{O}_{v}^{\times}$. The quadratic form $w_{\mathrm{sp}}$ can be made into the form $\left({ }^{2 I_{(n+1) / 2}}-2 I_{(n-1) / 2}\right)$ by the action of $\operatorname{GL}(n)_{k_{v}}$. Since $\pm 2 \in \mathcal{O}_{v}^{\times}, \widetilde{S}_{v}\left(w_{\mathrm{sp}}\right)=1$. Since $G_{k_{v}} w_{\mathrm{sp}} \neq G_{k_{v}} w_{\mathrm{rm}}, \widetilde{S}_{v}\left(w_{\mathrm{rm}}\right)=-1$.

We next assume that $n \geq 4$ is even. We continue to assume that $v \in \mathfrak{M}_{\mathrm{f}}$. We define a quaternary quadratic form $w_{\mathrm{dq}}^{0}$ as follows:

$$
w_{v, \mathrm{dq}}^{0}=\left(\begin{array}{lll}
A_{v, \text { in }} & & \\
& \pi_{v} A_{v, \text { in }}
\end{array}\right)
$$


where $A_{v, \text { in }}$ is the symmetric matrix in (4.5). It is easy to see that $w_{v, \mathrm{dq}}^{0}$ is anisotropic.

Remark 4.13. The index "dq" stands for "division quaternion algebra". The above $w_{v, \mathrm{dq}}^{0}$ corresponds to the norm of the unique division quaternion algebra over $k_{v}$ (see [22, pp. 142-149]).

Definition 4.14. Let $n \geq 4$ be even. We define $n$-ary quadratic forms $w_{v, \mathrm{dq}}, w_{v, \mathrm{sp}}, w_{v, \text { in }}$ and $w_{v,(\mathrm{rm}, j)}$ for $1 \leq j \leq \lambda_{v}$ as follows:

$$
\begin{aligned}
& w_{v, \mathrm{sp}}=\left(\begin{array}{lll}
H & & \\
& \ddots & \\
& & H
\end{array}\right), \quad w_{v, \text { in }}=\left(\begin{array}{cccc}
H & & & \\
& \ddots & & \\
& & H & \\
& & & A_{v, \text { in }}
\end{array}\right), \\
& w_{v,(\mathrm{rm}, j)}=\left(\begin{array}{cccc}
H & & & \\
& \ddots & & \\
& & H & \\
& & A_{v,(\mathrm{rm}, j)}
\end{array}\right), \quad w_{v, \mathrm{dq}}=\left(\begin{array}{llll}
H & & \\
& \ddots & & \\
& & H & \\
& & & w_{v, \mathrm{dq}}^{0}
\end{array}\right) .
\end{aligned}
$$

We put $w_{v, \text { in }}^{0}=A_{v, \text { in }}$ and $w_{v,(\mathrm{rm}, j)}^{0}=A_{v,(\mathrm{rm}, j)}$ for $1 \leq j \leq \lambda_{v}$. If there is no confusion, we write $w_{\mathrm{in}}^{0}, w_{(\mathrm{rm}, j)}^{0}, w_{\mathrm{dq}}^{0}$ and $w_{\mathrm{in}}, w_{(\mathrm{rm}, j)}, w_{\mathrm{dq}}$ (we did not define $\left.w_{v, \mathrm{sp}}^{0}\right)$.

In the above definition, the index "in" stands for "inert". From now on, we shall use the symbol $\dot{i}$ (or $\dot{1}_{1}$, etc.,) for the index of the representatives. So $\dot{\mathrm{i}}=\mathrm{sp}$ or $\mathrm{rm}$ if $n$ is odd, and $\dot{\mathrm{i}}=\mathrm{sp}$, in, $(\mathrm{rm}, j)$ or dq if $n$ is even.

Proposition 4.16. Let $n \geq 4$ be even and $v \in \mathfrak{M}_{\mathrm{f}}$. Then

$$
\left\{w_{\mathrm{sp}}, w_{\mathrm{in}}, w_{(\mathrm{rm}, 1)}, \ldots, w_{\left(\mathrm{rm}, \lambda_{v}\right)}, w_{\mathrm{dq}}\right\}
$$

is a complete set of representatives for $G_{k_{v}} \backslash V_{k_{v}}^{\mathrm{ss}}$.

Proof. We first prove that $G_{k_{v}} w_{\dot{\mathrm{i}}_{1}} \neq G_{k_{v}} w_{\dot{\mathrm{i}}_{2}}$ for all $\dot{\mathrm{i}}_{1} \neq \dot{\mathbb{1}}_{2}$. Since $n$ is even, $d_{v}$ is invariant under the action of $G_{k_{v}}$. It is easy to see that

$$
\begin{aligned}
& d_{v}\left(w_{\mathrm{dq}}\right) \equiv d_{v}\left(w_{\mathrm{sp}}\right) \equiv(-1)^{\frac{n}{2}}, \quad d_{v}\left(w_{\mathrm{in}}\right) \equiv(-1)^{\frac{n}{2}-1} \operatorname{det} A_{v, \mathrm{in}}, \\
& d_{v}\left(w_{(\mathrm{rm}, j)}\right) \equiv(-1)^{\frac{n}{2}-1} \operatorname{det} A_{v,(\mathrm{rm}, j)}
\end{aligned}
$$

for $1 \leq j \leq \lambda_{v}$. So

$$
d_{v}\left(w_{\dot{\mathrm{i}}_{1}}\right) \not \equiv d_{v}\left(w_{\dot{\mathrm{i}}_{2}}\right)\left(\dot{\mathrm{i}}_{1} \neq \dot{\mathrm{i}}_{2}\right)
$$


except for $\left(\dot{\mathbb{i}}_{1}, \dot{\mathbb{1}}_{2}\right)=(\mathrm{sp}, \mathrm{dq})$. Therefore,

$$
G_{k_{v}} w_{\dot{\mathrm{i}}_{1}} \neq G_{k_{v}} w_{\dot{\mathrm{i}}_{2}}\left(\dot{\mathrm{i}}_{1} \neq \dot{\mathrm{i}}_{2}\right)
$$

except for $\left(\dot{\mathbb{1}}_{1}, \dot{\mathbb{1}}_{2}\right)=(\mathrm{sp}, \mathrm{dq})$. It is easy to see that the Witt index of $w_{\mathrm{dq}}$ (resp. $\left.w_{\mathrm{sp}}\right)$ is $(n-4) / 2(\operatorname{resp} . n / 2)$. So $G_{k_{v}} w_{\mathrm{dq}} \neq G_{k_{v}} w_{\mathrm{sp}}$. Thus, we have $G_{k_{v}} w_{\dot{\mathrm{i}}_{1}} \neq G_{k_{v}} w_{\dot{\mathrm{i}}_{2}}$ for all $\dot{\mathrm{i}}_{1} \neq \dot{\mathrm{i}}_{2}$.

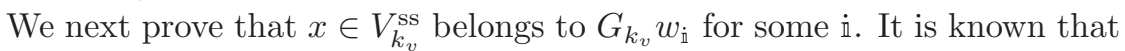
an $n$-ary quadratic form $x$ over $k_{v}$ is isotropic if $v \in \mathfrak{M}_{\mathrm{f}}$ and $n \geq 5$ (see (63:19) [22, p. 170]). Therefore, the Witt index of $x$ is $n / 2,(n-2) / 2$ or $(n-4) / 2$. If it is $n / 2, x$ clearly belongs to $G_{k_{v}} w_{\text {sp }}$. Let $\mu_{v}$ be a non-square unit whose square root generates the unramified quadratic extension of $k_{v}$. It is known that every anisotropic quaternary quadratic form can be made into the following form:

$$
\left(\begin{array}{llll}
1 & & & \\
& -\mu_{v} & & \\
& & \pi_{v} & \\
& & & -\mu_{v} \pi_{v}
\end{array}\right)
$$

by the action of GL(4) $k_{v}$ (see [22, p. 169]). So the above quadratic form belongs to the GL(4) $k_{v}$-orbit of $w_{\mathrm{dq}}^{0}$. If the Witt index of $x$ is $(n-4) / 2$ then $x$ is in the orbit of (4.17). Since $w_{\mathrm{dq}}^{0}$ is also in the orbit of (4.17), $x$ is in the orbit of $w_{\mathrm{dq}}$.

Suppose that the Witt index of $x$ is $(n-2) / 2$. Then by the action of $G_{k_{v}}$, $x$ belongs to the $G_{k_{v}}$-orbit of a quadratic form in the following form:

$$
\left(\begin{array}{llll}
H & & & \\
& \ddots & & \\
& & H & \\
& & & A
\end{array}\right)
$$

where $A$ corresponds to an anisotropic binary quadratic form. Note that $A$ belongs to the $\left(k_{v}^{\times} \times \mathrm{GL}(2)_{k_{v}}\right)$-orbit of one of the symmetric matrices $A_{v,(\mathrm{rm}, j)}$ in (4.5). If $\alpha \in k_{v}^{\times}$is a scalar then $\alpha H \in \mathrm{GL}(2)_{k_{v}} H$. Therefore, $x$ belongs to $G_{k_{v}} w_{\text {in }}$ or $G_{k_{v}} w_{(\mathrm{rm}, j)}$ for some $1 \leq j \leq \lambda_{v}$.

This completes the proof of the proposition.

We now consider an arbitrary $n \geq 2$ (but still assume that $v \in \mathfrak{M}_{\mathrm{f}}$ ). Let $w_{v, \mathrm{i}}$ be one of the representatives in (4.10), (4.15). We denote the Witt index of $w_{v, \text { i }}$ by $m$ and put $m_{0}=n-2 m$. Multiplying an element of $\mathrm{GL}(1)_{\mathcal{O}_{v}} \times \mathrm{GL}(n)_{\mathcal{O}_{v}}$, 
$w_{v, \mathrm{i}}$ can be made into the following form:

$$
w_{v, \mathrm{i}}^{\prime}= \begin{cases}\left(\begin{array}{cc}
0 & -I_{\frac{n}{2}} \\
-I_{\frac{n}{2}} & 0
\end{array}\right) & m_{0}=0, \\
\left(\begin{array}{ccc}
0 & 0 & -I_{m} \\
0 & w_{v, \mathrm{i}}^{0} & 0 \\
-I_{m} & 0 & 0
\end{array}\right) & m_{0} \neq 0 .\end{cases}
$$

Clearly $G_{k_{v}} w_{v, \mathrm{i}}=G_{k_{v}} w_{v, \mathrm{i}}^{\prime}$.

If $v \in \mathfrak{M}_{\mathrm{f}}$ then we call $w_{v, \mathrm{i}}$ and $w_{v, \dot{\mathrm{i}}}^{\prime}$ respectively the standard orbital representative and the alternative orbital representative (or simply the standard representative and the alternative representative) for $G_{k_{v}} \backslash V_{k_{v}}^{\text {ss }}$. We use the standard orbital representatives and the alternative orbital representatives depending on purposes. If there is no confusion, we write $w_{\mathfrak{i}}^{\prime}$ instead of $w_{v, \mathbb{1}}^{\prime}$.

Definition 4.19. Let $v \in \mathfrak{M}_{\mathrm{f}}$ and $x \in V_{k_{v}}^{\mathrm{ss}}$. If $n$ is odd, we say that $x$ is unramified (resp. ramified) if $x \in G_{k_{v}} w_{\mathrm{sp}}$ (resp. $x \in G_{k_{v}} w_{\mathrm{rm}}$ ). If $n$ is even, we say that $x$ is unramified (resp. ramified) if $x \in G_{k_{v}} w_{\text {sp }}$ or $x \in G_{k_{v}} w_{\text {in }}$ (resp. $x \in G_{k_{v}} w_{(\mathrm{rm}, j)}$ or $\left.x \in G_{k_{v}} w_{\mathrm{dq}}\right)$.

We consider this notion only for finite places.

If $n$ is even and $x \in V_{k_{v}}^{\text {ss }}$, then we can associate the quadratic extension $k_{v}\left(\sqrt{(-1)^{n / 2} d_{v}(x)}\right) / k_{v}$. There is a notion of ramification for quadratic extensions. If $x=w_{v, \mathrm{dq}}$ then $x$ is ramified according to Definition 4.19. However, the extension $k_{v}\left(\sqrt{(-1)^{n / 2} d_{v}(x)}\right) / k_{v}$ is trivial and so it is unramified. So the notion of ramification in the sense of Definition 4.19 does not coincide with the notion of ramification of the extension $k_{v}\left(\sqrt{(-1)^{n / 2} d_{v}(x)}\right) / k_{v}$.

We next consider the case $v \in \mathfrak{M}_{\infty}$. Let $r=[n / 2]$.

Definition 4.20. Let $n \geq 2$. If $v \in \mathfrak{M}_{\mathbb{C}}$ then we define $n$-ary quadratic forms $w_{v \text {,sp }}$ and $w_{v, \text { sp }}^{\prime}$ respectively as follows:

$$
w_{v, \mathrm{sp}}=I_{n}, \quad w_{v, \mathrm{sp}}^{\prime}=\left(\begin{array}{cr}
I_{n-2 r} & -I_{r} \\
-I_{r} &
\end{array}\right) .
$$

If $v \in \mathfrak{M}_{\mathbb{R}}$ then we define $n$-ary quadratic forms $w_{v, \mathrm{i}}$ and $w_{v, \mathbb{i}}^{\prime}$ for $0 \leq \dot{\mathrm{i}} \leq r$ respectively as follows:

$$
w_{v, \mathrm{i}}=\left(\begin{array}{cc}
-I_{\dot{\mathrm{i}}} & 0 \\
0 & I_{n-\mathrm{i}}
\end{array}\right), \quad w_{v, \mathrm{i}}^{\prime}=\left(\begin{array}{c}
I_{n-2 \mathrm{i}} \\
-I_{\mathrm{i}}
\end{array}\right) .
$$


If there is no confusion, we write $w_{\mathrm{sp}}, w_{\mathrm{sp}}^{\prime}$ and $w_{\mathrm{i}}, w_{\mathfrak{\mathrm { i }}}^{\prime}$.

It is easy to see that $G_{k_{v}} w_{v, \mathrm{i}}=G_{k_{v}} w_{v, \mathrm{i}}^{\prime}$ for all $\dot{\mathrm{i}}$ and $v \in \mathfrak{M}_{\infty}$. Clearly, $G_{\mathbb{C}} \backslash V_{\mathbb{C}}^{\text {ss }}$ consists of a single orbit and so we can choose $w_{v, \mathrm{sp}}$ or $w_{v, \mathrm{sp}}^{\prime}$ as the representative. It is well-known that either of $\left\{w_{0}, \cdots, w_{r}\right\}$ or $\left\{w_{0}^{\prime}, \cdots, w_{r}^{\prime}\right\}$ is a complete set of representatives for $G_{\mathbb{R}} \backslash V_{\mathbb{R}}^{\mathrm{ss}}$.

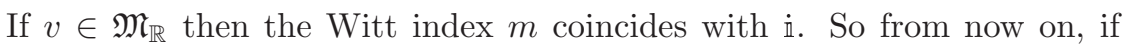
$v \in \mathfrak{M}_{\mathbb{R}}$ then we shall use $\dot{\mathrm{i}}$ instead of $m$ to express various matrices related to orbits.

Remark 4.21. Clearly, $\left|P\left(w_{v, \mathrm{i}}\right)\right|_{v}=\left|P\left(w_{v, \mathrm{i}}^{\prime}\right)\right|_{v}=|1 / 2|_{v}$ if $n$ is odd, and $\left|P\left(w_{v, \mathrm{i}}\right)\right|_{v}=\left|P\left(w_{v, \mathrm{i}}^{\prime}\right)\right|_{v}=1$ if $n$ is even. It would have been desirable to choose representatives $x$ so that $|P(x)|_{v}=1$. However, we shall consider the Iwasawa decompositions of the stabilizers in Part II, and for that purpose, it will turn out to be more convenient to choose the above representatives $w_{v, \mathrm{i}}$ or $w_{v, \mathrm{i}}^{\prime}$.

For $v \in \mathfrak{M}_{\infty}$, we call $w_{v, \mathrm{i}}$ and $w_{v, \mathrm{i}}^{\prime}$ respectively a standard orbital representative and an alternative orbital representative for $G_{k_{v}} \backslash V_{k_{v}}^{\mathrm{ss}}$.

Let $r=[n / 2]$ as above. We define $\sigma_{v, \text { sp }}$ for $v \in \mathfrak{M}_{\mathbb{C}}$ and $\sigma_{v, \text { i }}$ for $v \in \mathfrak{M}_{\mathbb{R}}$ as follows:

$$
\sigma_{v, \mathrm{sp}}=\left(\begin{array}{ccc}
\frac{\sqrt{-2}}{2} I_{r} & 0_{r, n-2 r} & \frac{\sqrt{2}}{2} I_{r} \\
0_{n-2 r, r} & I_{n-2 r} & 0_{n-2 r, r} \\
\frac{\sqrt{-2}}{2} I_{r} & 0_{r, n-2 r} & -\frac{\sqrt{2}}{2} I_{r}
\end{array}\right), \quad \sigma_{v, \mathrm{i}}=\left(\begin{array}{ccc}
\frac{\sqrt{2}}{2} I_{\mathrm{i}} & 0_{\mathrm{i}, n-2 \mathrm{i}} & \frac{\sqrt{2}}{2} I_{\dot{\mathrm{i}}} \\
0_{n-2 \mathrm{i}, \mathrm{i}} & I_{n-2 \mathrm{i}} & 0_{n-2 \mathrm{i}, \mathrm{i}} \\
\frac{\sqrt{2}}{2} I_{\mathrm{i}} & 0_{\mathrm{i}, n-2 \mathfrak{\mathrm { i }}}-\frac{\sqrt{2}}{2} I_{\mathrm{i}}
\end{array}\right) .
$$

Then $\sigma_{v, \mathrm{sp}} w_{v, \mathrm{sp}}=w_{v, \mathrm{sp}}^{\prime}$ for $v \in \mathfrak{M}_{\mathbb{C}}$, and $\sigma_{v, \mathrm{i}} w_{v, \mathrm{i}}=w_{v, \mathrm{i}}^{\prime}$ for $v \in \mathfrak{M}_{\mathbb{R}}$.

We need the following proposition for later purposes.

Proposition 4.22. Let $n=2 r \geq 2$ be even. If $v \in \mathfrak{M}$ and $x \in V_{k_{v}}^{\mathrm{ss}}$ is a standard representative then there exists an element $\tau_{x}$ of $G_{x k_{v}}$ not in $G_{x k_{v}}^{\circ}$ whose order is two. If $v \in \mathfrak{M}_{\mathrm{f}}$ then one can choose $\tau_{x}$ in $G_{x k_{v}} \cap K_{v}$.

Proof. The point of the first statement is that we can take $\tau_{x}$ rationally over $k_{v}$.

We first consider the case $v \in \mathfrak{M}_{\mathrm{f}}$. The statements are clearly satisfied for $w_{v, \text { sp }}$ and so we only consider $w_{v, \text { i }}$ for $\dot{\mathrm{i}}=\mathrm{in},(\mathrm{rm}, j)$ and dq (if $\left.n \geq 4\right)$. Let $\alpha_{j}, \beta_{j}$ be the roots of $p_{j}(z)$. We put

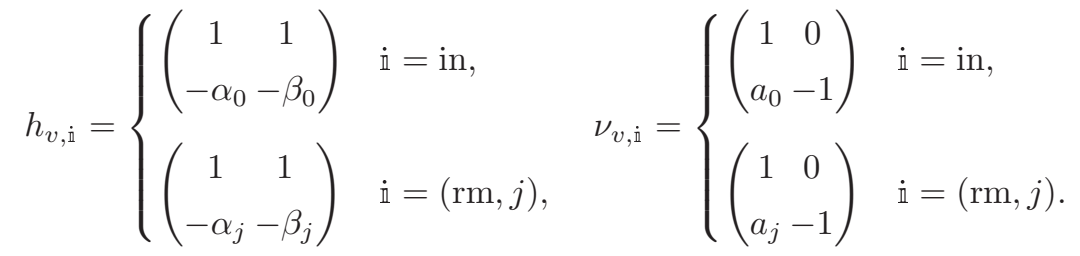


Then $h_{v, \text { in }} H^{t} h_{v, \dot{\mathrm{i}}}=A_{v, \dot{\mathrm{i}}}, h_{v, \mathrm{i}} H h_{v, \mathfrak{\mathrm { i }}}^{-1}=\nu_{v, \mathrm{i}}$ for $\dot{\mathrm{i}}=\mathrm{in},(\mathrm{rm}, j)$ by straightforward computations.

Let $\tau$ be as in (3.13). If we put

$$
\begin{aligned}
& g(v, \dot{\mathrm{i}})=\left(\begin{array}{ccc}
I_{n-2} & \\
& h_{v, \mathrm{i}}
\end{array}\right), \tau_{v, \mathrm{i}}=\left(\begin{array}{cc}
I_{n-2} & \\
& \nu_{v, \mathrm{i}}
\end{array}\right) \dot{\mathrm{i}}=\mathrm{in},(\mathrm{rm}, j), \\
& g(v, \mathrm{dq})=\left(\begin{array}{ccc}
I_{n-4} & & \\
& h_{v, \text { in }} & \\
& & \sqrt{\pi_{v}} h_{v, \text { in }}
\end{array}\right), \tau_{v, \mathrm{dq}}=\left(\begin{array}{cc}
I_{n-2} & \\
& \nu_{v, \text { in }}
\end{array}\right),
\end{aligned}
$$

then for $\dot{\mathrm{i}}=\mathrm{in},(\mathrm{rm}, j)$ and $\mathrm{dq}$,

$$
w_{v, \dot{\mathrm{i}}}=g(v, \dot{\mathrm{i}}) w, \quad g(v, \dot{\mathrm{i}}) \tau g(v, \dot{\mathrm{i}})^{-1}=\tau_{v, \dot{\mathrm{i}}} .
$$

We pointed out that $\tau$ is an outer automorphism of $\operatorname{GO}(w)^{\circ}$ in the proof of Lemma 3.11. Since $\tau_{v, \text { i }} \in K_{v}$, this completes the proof of the proposition for $v \in \mathfrak{M}_{\mathrm{f}}$.

We next consider the case $v \in \mathfrak{M}_{\infty}$. If $v \in \mathfrak{M}_{\mathbb{C}}$ then the proposition is obvious since $V_{\mathbb{C}}^{\text {ss }}$ consists of a single orbit, and so we assume that $v \in \mathfrak{M}_{\mathbb{R}}$.

Let $0 \leq \dot{i} \leq r$. We put

$$
S=\frac{1}{\sqrt{2}}\left(\begin{array}{cc}
1 & 1 \\
-\sqrt{-1} \sqrt{-1}
\end{array}\right), \quad g(v, \dot{\mathrm{i}})=\left(\begin{array}{cccc}
I_{2 \mathrm{i}} & & & \\
& S & & \\
& & \ddots & \\
& & & S
\end{array}\right) .
$$

Since $S H^{t} S=I_{2}$, the signature of $g(v, \dot{\mathrm{i}}) w$ is $(n-\dot{\mathrm{i}}, \dot{\mathrm{i}})$. So $g(v, \dot{\mathrm{i}}) w \in G_{k_{v}} w_{v, \mathrm{i}}$. Therefore, it is enough to prove the statement of the proposition for $x=$ $g(v, \dot{\mathrm{I}}) w$.

By simple computations,

$$
g(v, \dot{\mathrm{I}}) \tau g(v, \grave{\mathrm{I}})^{-1}=a(1, \cdots, 1,-1) \in G_{x k_{v}} .
$$

This completes the proof of the proposition.

\section{$\S 5 . \quad$ The Discriminants of Orbits}

The main purpose of this section is to define the notion of discriminant for orbits both locally and globally. We continue to assume that $k$ is a number field. We use notations such as $\lambda_{v}$ in (4.1), etc., also in this section. 
We first define some notations concerning $G$. Let $T_{n} \subseteq \mathrm{GL}(n)$ be the set of diagonal matrices and $N_{n} \subseteq \mathrm{GL}(n)$ the set of lower triangular matrices whose diagonal entries are 1 . Then $B_{n}=T_{n} N_{n}$ is a Borel subgroup of GL $(n)$.

We define the classical orthogonal group and the special orthogonal group as follows:

$$
\mathrm{O}(n)=\left\{X \in \mathrm{GL}(n)_{\mathbb{R}} \mid X^{t} X=I_{n}\right\}, \mathrm{SO}(n)=\left\{X \in \mathrm{SL}(n)_{\mathbb{R}} \mid X^{t} X=I_{n}\right\} .
$$

We define the classical unitary group $\mathrm{U}(n)$ by $\mathrm{U}(n)=\left\{X \in \mathrm{GL}(n)_{\mathbb{C}} \mid X X^{*}=\right.$ $\left.I_{n}\right\}$ where $X^{*}={ }^{t} \bar{X}$ is the conjugate transpose of $X$. We define a subgroup $K_{n, v}$ of $\operatorname{GL}(n)_{k_{v}}$ as follows:

$$
K_{n, v}= \begin{cases}\mathrm{GL}(n)_{\mathcal{O}_{v}} & v \in \mathfrak{M}_{\mathrm{f}} \\ \mathrm{O}(n) & v \in \mathfrak{M}_{\mathbb{R}} \\ \mathrm{U}(n) & v \in \mathfrak{M}_{\mathbb{C}}\end{cases}
$$

It is known that $\mathrm{GL}(n)_{k_{v}}$ contains $K_{n, v}$ as a maximal compact subgroup and has the decomposition $\operatorname{GL}(n)_{k_{v}}=K_{n, v} B_{n k_{v}}$. We define a subgroup $K_{v}$ of $G_{k_{v}}$ as follows:

$$
K_{v}=K_{1, v} \times K_{n, v} .
$$

For the rest of this section we assume that $v \in \mathfrak{M}_{\mathrm{f}}$.

Definition 5.2. $\quad$ For each $v \in \mathfrak{M}_{\mathrm{f}}$, we define an integral structure on $V$ by

$$
V_{\mathcal{O}_{v}}=\left\{\left(\begin{array}{cccc}
2 x_{11} & x_{12} & \cdots & x_{1 n} \\
x_{12} & 2 x_{22} & \ddots & \vdots \\
\vdots & \ddots & \ddots & x_{n-1 n} \\
x_{1 n} & \cdots & x_{n-1 n} & 2 x_{n n}
\end{array}\right) \in V_{k_{v}} \mid x_{i j} \in \mathcal{O}_{v}, 1 \leq i \leq j \leq n\right\} .
$$

Lemma 5.3. We have $P(x) \in \mathbb{Z}[x]$.

Proof. The statement is obvious if $n$ is even. So we assume that $n$ is odd. We prove the lemma by induction on odd $n$. The case $n=1$ is obvious and so we assume that $n \geq 3$ is odd.

Let $M_{11}$ be the matrix obtained by removing the first row and the first column of $x$, and $M_{i j}^{\prime}$ the matrix obtained by removing the first and the $i$-th rows and the first and the $j$-th columns of $x$ for $2 \leq i \leq j \leq n$. We consider 
the cofactor expansion of $x$ with respect to the first row and then consider the cofactor expansion with respect to the first column. Since $x$ is symmetric,

$$
\operatorname{det} x=2 x_{11} \operatorname{det} M_{11}-\sum_{i=2}^{n} x_{1 i}^{2} \operatorname{det} M_{i i}^{\prime}+2 \sum_{2 \leq i<j \leq n}(-1)^{i+j+1} \operatorname{det} M_{i j}^{\prime} .
$$

Since $M_{i i}^{\prime}$ is a symmetric matrix, $\operatorname{det} M_{i i}^{\prime}$ is divisible by 2 as a polynomial in $\mathbb{Z}[x]$ by induction. So $P(x)=2^{-1} \operatorname{det} M_{x} \in \mathbb{Z}[x]$.

The point $w$ is "universally generic" in the sense of Section 2 [17]. So we may apply Proposition 1 [17] to the present situation. However, since the proof is very easy, we chose to include the above explicit proof.

Definition 5.4. We define the discriminant $\Delta_{x, v}$ of $x \in V_{k_{v}}^{\mathrm{ss}}$ for $v \in \mathfrak{M}_{\mathrm{f}}$ as follows:

$$
\Delta_{x, v}=\min \left\{q_{v}^{\operatorname{ord}_{v}\left(P\left(x^{\prime}\right)\right)} \mid x^{\prime} \in G_{k_{v}} x \cap V_{\mathcal{O}_{v}}\right\} .
$$

Remark 5.5. The discriminant of $x$ depends only on the orbit $G_{k_{v}} x$. We defined the above discriminant so that the discriminant of quadratic form $w_{\mathrm{sp}}$ in $\S 4$ is 1 for all $n$. (see Proposition 5.23 below).

Let $x \in V_{k}^{\text {ss }}$. It is easy to see that $x \in V_{\mathcal{O}_{v}}$ and $\operatorname{det} x \in \mathcal{O}_{v}^{\times}$for all but finitely many $v \in \mathfrak{M}_{\mathrm{f}}$. Therefore, $\Delta_{x, v}=q_{v}^{\operatorname{ord}_{v}(P(x))}=1$ for all but finitely many $v \in \mathfrak{M}_{\mathrm{f}}$. Thus, we can define the global discriminant as follows.

Definition 5.6. We define the discriminant $\Delta_{x}$ of $x \in V_{k}^{\text {ss }}$ as follows:

$$
\Delta_{x}=\prod_{v \in \mathfrak{M}_{\mathrm{f}}} \Delta_{x, v} .
$$

For the rest of this section, we determine the values of discriminants for standard orbital representatives.

Let $x \in V_{k_{v}}^{\mathrm{ss}}$. We first state some conditions on $x$.

Condition 5.7. If $g \in G_{k_{v}}$ and $g x \in V_{\mathcal{O}_{v}}$, then $\operatorname{ord}_{v}(\chi(g)) \geq 0$.

Condition 5.8. If $g \in G_{k_{v}}, g x \in V_{\mathcal{O}_{v}}$ and $\chi(g) \in \mathcal{O}_{v}^{\times}$, then $g \in$ $K_{v} G_{x k_{v}}$.

Condition 5.9. If $g=\left(1, g_{1}\right) \in G_{k_{v}}, g x \in V_{\mathcal{O}_{v}}$ and $\chi(g) \in \mathcal{O}_{v}^{\times}$, then $g_{1} \in K_{n, v}$.

Condition 5.10. If $x[v] \in \mathcal{O}_{v}$ for a row vector $v \in k_{v}^{n}$, then $v \in \mathcal{O}_{v}^{n}$. 
We do not use Conditions 5.8, 5.9 and 5.10 to determine the discriminants. In $\S 8$, we shall use Condition 5.8 to prove that the measures on $\operatorname{GO}(x)_{\AA}^{\circ}$ and $\mathrm{SO}(x)_{\mathbb{A}}$ for $x \in V_{k}^{\mathrm{ss}}$ are well-defined. Moreover, in Part III, for even $n \geq 2$, we shall use Condition 5.8 to prove that the constant terms of the $q$-expansions of local orbital zeta functions are 1 except for finitely many places.

Conditions 5.9 and 5.10 may be useful in the proof of the Iwasawa decompositions of $\operatorname{GO}\left(w_{\dot{\mathrm{i}}}^{\prime}\right)_{k_{v}}^{\circ}$ and $\mathrm{SO}\left(w_{\dot{\mathrm{i}}}^{\prime}\right)_{k_{v}}$. In $\S 8$, we shall define measures on $\operatorname{GO}\left(w_{\dot{\mathrm{i}}}^{\prime}\right)_{k_{v}}$ and $\mathrm{SO}\left(w_{\dot{\mathrm{i}}}^{\prime}\right)_{k_{v}}$. The Iwasawa decompositions tell us that the measures in $\S 8$ are natural in some sense. The Iwasawa decompositions of these groups are well-known. For example, Satake [24] discusses the proof of the Iwasawa decomposition of $\mathrm{GO}\left(w_{\dot{\mathrm{n}}}^{\prime}\right)_{k_{v}}^{\circ}$ in [24, pp. 50-53]. However, the notation and the formulation in [24] are not totally compatible with our paper. So we briefly discuss the Iwasawa decompositions in $\S 7$.

Even though Conditions 5.8, 5.9 and 5.10 are not directly related to the notion of discriminant, which is the topic of this section, we can verify that these conditions and Condition 5.7 are satisfied for $w_{\dot{\mathrm{i}}}$ by the same argument. So we consider them in this section for convenience.

Suppose that $x \in V_{\mathcal{O}_{v}}$ satisfies Condition 5.7. If $g \in G_{k_{v}}$ and $g x \in V_{\mathcal{O}_{v}}$, then

$$
\operatorname{ord}_{v}(P(g x))=\operatorname{ord}_{v}(\chi(g))+\operatorname{ord}_{v}(P(x)) \geq \operatorname{ord}_{v}(P(x))
$$

since $P(g x)=\chi(g) P(x)$. Since

$$
\operatorname{ord}_{v}(P(x))=\min \left\{\operatorname{ord}_{v}\left(P\left(x^{\prime}\right)\right) \mid x^{\prime} \in G_{k_{v}} x \cap V_{\mathcal{O}_{v}}\right\},
$$

$\Delta_{x, v}=q_{v}^{\operatorname{ord}_{v}(P(x))}$. Thus, we can determine the value of $\Delta_{x, v}$ using Condition 5.7 .

The following observation may be useful to verify that Conditions 5.7, 5.8 and 5.9 are satisfied for $x \in V_{\mathcal{O}_{v}}^{\text {ss }}$.

Suppose that we try to verify that Condition 5.7 is satisfied for $x \in V_{k_{v}}^{\text {ss }}$. If $g \in G_{k_{v}}$ and $g x \in V_{\mathcal{O}_{v}}$, then we may replace $g$ by $\kappa g h$ where $\kappa \in K_{n, v}$ and $h \in G_{x k_{v}}$. For, $\kappa g h$ satisfies the conditions $\kappa g h x \in V_{\mathcal{O}_{v}}$ and

$$
\chi(g)=\chi(\kappa)^{-1} \chi(\kappa g h), \quad \chi(\kappa) \in \mathcal{O}_{v}^{\times} .
$$

Similarly, it suffices to verify that $\kappa g h \in K_{v} G_{x k_{v}}$ (resp. $\kappa g \in K_{n, v}$ ) for some $\kappa \in K_{n, v}, h \in G_{x k_{v}}$ in order to prove that $x \in V_{k_{v}}^{\text {ss }}$ satisfies Condition 5.8 (resp. Condition 5.9).

We first consider the case where $n$ is odd and the Witt index is 0 .

Lemma 5.11. Both $w_{\mathrm{sp}}^{0}$ and $w_{\mathrm{rm}}^{0}$ in (4.8) satisfy Conditions 5.7, 5.8, 
5.9 and 5.10. Moreover

$$
\Delta_{w_{\mathrm{sp}}^{0}, v}=1, \quad \Delta_{w_{\mathrm{rm}}^{0}, v}=q_{v}
$$

Proof. Since the statement holds clearly for $w_{\mathrm{sp}}^{0}$, we only consider $w_{\mathrm{rm}}^{0}$.

Let $n=3$. We prove that $w_{\text {rm }}^{0}$ satisfies Condition 5.10. Suppose that $v=\left(u_{1}, u_{2}, u_{3}\right) \in k_{v}^{3}$ satisfies the condition

$$
w_{\mathrm{rm}}^{0}[v]=u_{1}^{2}+a_{0} u_{1} u_{2}+b_{0} u_{2}^{2}+\pi_{v} u_{3}^{2} \in \mathcal{O}_{v} .
$$

Since $\operatorname{ord}_{v}\left(u_{1}^{2}+a_{0} u_{1} u_{2}+b_{0} u_{2}^{2}\right)$ is even and $\operatorname{ord}_{v}\left(\pi_{v} u_{3}^{2}\right)$ is odd for any

$$
u_{1}, u_{2}, u_{3} \in k_{v}, u_{1}^{2}+a_{0} u_{1} u_{2}+b_{0} u_{2}^{2}, \pi_{v} u_{3}^{2} \in \mathcal{O}_{v} .
$$

Thus, $u_{1}, u_{2}, u_{3} \in \mathcal{O}_{v}$.

We now prove that $w_{\mathrm{rm}}^{0}$ satisfies Conditions 5.7, 5.8 and 5.9. For three row vectors $v_{1}, v_{2}, v_{3} \in k_{v}^{3}$ and $t_{0} \in k_{v}^{\times}$, we put

$$
g_{1}=\left(\begin{array}{l}
v_{1} \\
v_{2} \\
v_{3}
\end{array}\right), \quad g=\left(t_{0}, g_{1}\right), \quad g w_{\mathrm{rm}}^{0}=\left(\begin{array}{ccc}
2 f_{1} & f_{2} & f_{3} \\
f_{2} & 2 f_{4} & f_{5} \\
f_{3} & f_{5} & 2 f_{6}
\end{array}\right) .
$$

It is easy to see that

$$
f_{1}=t_{0} w_{\mathrm{rm}}^{0}\left[v_{1}\right], \quad f_{4}=t_{0} w_{\mathrm{rm}}^{0}\left[v_{2}\right], \quad f_{6}=t_{0} w_{\mathrm{rm}}^{0}\left[v_{3}\right] .
$$

We first prove that $w_{\mathrm{rm}}^{0}$ satisfies Condition 5.9. Suppose that $t_{0}=1$, $g w_{\mathrm{rm}}^{0} \in V_{\mathcal{O}_{v}}$ and $\chi(g) \in \mathcal{O}_{v}^{\times}$. Multiplying an element of $K_{3, v}$ from the left, we may assume that $g_{1}$ is in the following form:

$$
\left(\begin{array}{ccc}
t_{1} & 0 & 0 \\
0 & t_{2} & 0 \\
0 & 0 & t_{3}
\end{array}\right)\left(\begin{array}{ccc}
1 & 0 & 0 \\
u_{1} & 1 & 0 \\
u_{2} & u_{3} & 1
\end{array}\right)=\left(\begin{array}{ccc}
t_{1} & 0 & 0 \\
t_{2} u_{1} & t_{2} & 0 \\
t_{3} u_{2} & t_{3} u_{3} & t_{3}
\end{array}\right)
$$

where $t_{1}, t_{2}, t_{3} \in k_{v}^{\times}$and $u_{1}, u_{2}, u_{3} \in k_{v}$. Then $v_{1}=\left(t_{1}, 0,0\right), v_{2}=\left(t_{2} u_{1}, t_{2}, 0\right)$, $v_{3}=\left(t_{3} u_{2}, t_{3} u_{3}, t_{3}\right)$ and

$$
\begin{aligned}
& f_{1}=w_{\mathrm{rm}}^{0}\left[v_{1}\right]=t_{1}^{2}, \\
& f_{4}=w_{\mathrm{rm}}^{0}\left[v_{2}\right]=t_{2}^{2}\left(u_{1}^{2}+a_{0} u_{1}+b_{0}\right), \\
& f_{6}=w_{\mathrm{rm}}^{0}\left[v_{3}\right]=t_{3}^{2}\left(u_{2}^{2}+a_{0} u_{2} u_{3}+b_{0} u_{3}^{2}+\pi_{v}\right) .
\end{aligned}
$$

Since $f_{1}, f_{4}, f_{6} \in \mathcal{O}_{v}$ and $w_{\mathrm{rm}}^{0}$ satisfies Condition 5.10, $t_{1}, t_{2}, t_{3} \in \mathcal{O}_{v}$. Since $\chi\left(g_{1}\right)=t_{1}^{2} t_{2}^{2} t_{3}^{2} \in \mathcal{O}_{v}^{\times}, t_{1}, t_{2}, t_{3} \in \mathcal{O}_{v}^{\times}$. Since $t_{2} u_{1}, t_{3} u_{2}, t_{3} u_{3} \in \mathcal{O}_{v}, u_{1}, u_{2}, u_{3} \in$ $\mathcal{O}_{v}$. Thus, $g_{1} \in K_{3, v}$. 
We next prove that $w_{\mathrm{rm}}^{0}$ satisfies Condition 5.7. Suppose that $g w_{\mathrm{rm}}^{0} \in V_{\mathcal{O}_{v}}$. By multiplying an element of $K_{3, v}$ from the left and an element of $\widetilde{T}_{k_{v}} \subseteq G_{w_{\mathrm{rm}}^{0} k_{v}}$ from the right, we may assume that $g_{1}$ is in the form (5.12) and $t_{1}=1$. Then

$$
\begin{aligned}
& f_{1}=t_{0} w_{\mathrm{rm}}^{0}\left[v_{1}\right]=t_{0}, \\
& f_{4}=t_{0} w_{\mathrm{rm}}^{0}\left[v_{2}\right]=t_{0} t_{2}^{2}\left(u_{1}^{2}+a_{0} u_{1}+b_{0}\right), \\
& f_{6}=t_{0} w_{\mathrm{rm}}^{0}\left[v_{3}\right]=t_{0} t_{3}^{2}\left(u_{2}^{2}+a_{0} u_{2} u_{3}+b_{0} u_{3}^{2}+\pi_{v}\right) .
\end{aligned}
$$

Since $f_{1} \in \mathcal{O}_{v}, t_{0} \in \mathcal{O}_{v}$. It is easy to see that $t_{0} f_{4}=w_{\mathrm{rm}}^{0}\left[t_{0} v_{2}\right], t_{0} f_{6}=$ $w_{\mathrm{rm}}^{0}\left[t_{0} v_{3}\right]$. Since $w_{\mathrm{rm}}^{0}$ satisfies Condition 5.10, $t_{0} v_{2}=\left(t_{0} t_{2} u_{1}, t_{0} t_{2}, 0\right), t_{0} v_{3}=$ $\left(t_{0} t_{3} u_{2}, t_{0} t_{3} u_{3}, t_{0} t_{3}\right) \in \mathcal{O}_{v}^{3}$. So $t_{0} t_{2}, t_{0} t_{3} \in \mathcal{O}_{v}$. Since $\operatorname{ord}_{v}\left(u_{1}^{2}+a_{0} u_{1}+b_{0}\right) \leq 0$ and $f_{4}=t_{0} t_{2}^{2}\left(u_{1}^{2}+a_{0} u_{1}+b_{0}\right) \in \mathcal{O}_{v}, t_{0} t_{2}^{2} \in \mathcal{O}_{v}$. Since $\chi(g)=\left(t_{0} t_{2}^{2}\right)\left(t_{0} t_{3}\right)^{2}$, $\chi(g) \in \mathcal{O}_{v}$.

We next prove that $w_{\mathrm{rm}}^{0}$ satisfies Condition 5.8. Suppose that $g w_{\mathrm{rm}}^{0} \in V_{\mathcal{O}_{v}}$ and $\chi(g) \in \mathcal{O}_{v}^{\times}$. Similarly as above, we may assume that $g_{1}$ is in the form (5.12) with $t_{1}=1$ and $f_{1}, f_{4}, f_{6}$ are in the form (5.13). We already verified that

$$
t_{0}, t_{0} t_{2}, t_{0} t_{3}, t_{0} t_{2}^{2} \in \mathcal{O}_{v} .
$$

Since $f_{6} \in \mathcal{O}_{v}$ and $\operatorname{ord}_{v}\left(u_{2}^{2}+a_{0} u_{2} u_{3}+b_{0} u_{3}^{2}+\pi_{v}\right) \leq 1, \operatorname{ord}_{v}\left(t_{0} t_{3}^{2}\right) \geq-1$. Since

$$
\operatorname{ord}_{v}(\chi(g))=\operatorname{ord}_{v}\left(t_{0}^{2} t_{2}^{2}\right)+\operatorname{ord}_{v}\left(t_{0} t_{3}^{2}\right)=0
$$

and $\operatorname{ord}_{v}\left(t_{0}^{2} t_{2}^{2}\right) \geq 0$ is even, $\operatorname{ord}_{v}\left(t_{0}^{2} t_{2}^{2}\right)=\operatorname{ord}_{v}\left(t_{0} t_{3}^{2}\right)=0$. Since

$$
\operatorname{ord}_{v}(\chi(g))=\operatorname{ord}_{v}\left(t_{0} t_{2}^{2}\right)+\operatorname{ord}_{v}\left(t_{0}^{2} t_{3}^{2}\right)=0,
$$

$\operatorname{ord}_{v}\left(t_{0} t_{2}^{2}\right)=\operatorname{ord}_{v}\left(t_{0}^{2} t_{3}^{2}\right)=0$. Thus, $t_{0}, t_{2}, t_{3} \in \mathcal{O}_{v}^{\times}$. It is easy to see that $t_{0} f_{4}=w_{\mathrm{rm}}^{0}\left[t_{0} v_{2}\right], t_{0} f_{6}=w_{\mathrm{rm}}^{0}\left[t_{0} v_{3}\right]$. Since $t_{0} f_{4}, t_{0} f_{6} \in \mathcal{O}_{v}$ and $w_{\mathrm{rm}}^{0}$ satisfies Condition 5.10, $t_{0} v_{2}, t_{0} v_{3} \in \mathcal{O}_{v}^{3}$. Since $t_{0} \in \mathcal{O}_{v}^{\times}, v_{2}, v_{3} \in \mathcal{O}_{v}^{3}$. Thus, $g \in K_{v}$.

Since $\operatorname{ord}_{v}\left(P\left(w_{\mathrm{sp}}^{0}\right)\right)=0, \operatorname{ord}_{v}\left(P\left(w_{\mathrm{rm}}^{0}\right)\right)=1$ and $w_{\mathrm{sp}}^{0}$ and $w_{\mathrm{rm}}^{0}$ satisfy Condition 5.7, we have $\Delta_{w_{\mathrm{sp}}^{0}, v}=1$ and $\Delta_{w_{\mathrm{rm}}^{0}, v}=q_{v}$.

This completes the proof of the lemma.

We next consider the case where $n$ is even.

Lemma 5.14. All of $w_{\mathrm{in}}^{0}, w_{(\mathrm{rm}, j)}^{0}$ for $1 \leq j \leq \lambda_{v}$ and $w_{\mathrm{dq}}^{0}$ satisfy Conditions 5.7, 5.8, 5.9 and 5.10. Moreover

$$
\begin{array}{ll}
\Delta_{w_{\mathrm{in}}^{0}, v} & =1, \\
\Delta_{w_{(\mathrm{rm}, j)}^{0}, v} & =q_{v} \\
\Delta_{w_{\mathrm{dq}}^{0}, v} & =q_{v}^{2} .
\end{array}
$$


Proof. The argument for the case $w_{\text {in }}^{0}$ is similar to that for the case $w_{\mathrm{dq}}^{0}$. So we only consider $w_{(\mathrm{rm}, j)}^{0}$ and $w_{\mathrm{dq}}^{0}$.

Let $n=2$. We first prove that $w_{(\mathrm{rm}, j)}^{0}$ satisfies Condition 5.10. Suppose that $v=\left(u_{1}, u_{2}\right) \in k_{v}^{2}$ and that

$$
w_{(\mathrm{rm}, j)}^{0}[v]=u_{1}^{2}+a_{j} u_{1} u_{2}+b_{j} u_{2}^{2} \in \mathcal{O}_{v} .
$$

If $u_{2}=0$ then $u_{1} \in \mathcal{O}_{v}$. If $u_{2} \neq 0$ then

$$
w_{(\mathrm{rm}, j)}^{0}[v]=u_{2}^{2}\left(\left(u_{1} u_{2}^{-1}\right)^{2}+a_{j} u_{1} u_{2}^{-1}+b_{j}\right) \in \mathcal{O}_{v} .
$$

Note that

$$
\operatorname{ord}_{v}\left(\left(u_{1} u_{2}^{-1}\right)^{2}+a_{j} u_{1} u_{2}^{-1}+b_{j}\right) \leq 0
$$

if $\operatorname{ord}_{v}\left(u_{1} u_{2}^{-1}\right) \leq 0$ and that

$$
\operatorname{ord}_{v}\left(\left(u_{1} u_{2}^{-1}\right)^{2}+a_{j} u_{1} u_{2}^{-1}+b_{j}\right)=1
$$

if $\operatorname{ord}_{v}\left(u_{1} u_{2}^{-1}\right) \geq 1$. So $\operatorname{ord}_{v}\left(u_{2}^{2}\right) \geq 0$. Since $u_{2} \in \mathcal{O}_{v}$ and $u_{1}^{2}+a_{j} u_{1} u_{2}+b_{j} u_{2}^{2} \in$ $\mathcal{O}_{v}, u_{1} \in \mathcal{O}_{v}$

We now prove that $w_{(\mathrm{rm}, j)}^{0}$ satisfies Conditions 5.7, 5.8 and 5.9. For two row vectors $v_{1}, v_{2} \in k_{v}^{2}$ and $t_{0} \in k_{v}^{\times}$, we put

$$
g_{1}=\left(\begin{array}{l}
v_{1} \\
v_{2}
\end{array}\right), \quad g=\left(t_{0}, g_{1}\right), \quad g w_{(\mathrm{rm}, j)}^{0}=\left(\begin{array}{cc}
2 f_{1} & f_{2} \\
f_{2} & 2 f_{3}
\end{array}\right) .
$$

It is easy to see that

$$
f_{1}=t_{0} w_{(\mathrm{rm}, j)}^{0}\left[v_{1}\right], \quad f_{3}=t_{0} w_{(\mathrm{rm}, j)}^{0}\left[v_{2}\right] .
$$

We first prove that $w_{(\mathrm{rm}, j)}^{0}$ satisfies Condition 5.9. Suppose that $t_{0}=1$, $g w_{(\mathrm{rm}, j)}^{0} \in V_{\mathcal{O}_{v}}$ and $\chi(g) \in \mathcal{O}_{v}^{\times}$. Multiplying an element of $K_{2, v}$ from the left, we may assume that $g_{1}$ is in the following form:

$$
\left(\begin{array}{cc}
t_{1} & 0 \\
0 & t_{2}
\end{array}\right)\left(\begin{array}{cc}
1 & 0 \\
u_{1} & 1
\end{array}\right)=\left(\begin{array}{cc}
t_{1} & 0 \\
t_{2} u_{1} & t_{2}
\end{array}\right)
$$

where $t_{1}, t_{2} \in k_{v}^{\times}$and $u_{1} \in k_{v}$. Then $v_{1}=\left(t_{1}, 0\right), v_{2}=\left(t_{2} u_{1}, t_{2}\right)$ and

$$
\begin{aligned}
& f_{1}=w_{(\mathrm{rm}, j)}^{0}\left[v_{1}\right]=t_{1}^{2}, \\
& f_{3}=w_{(\mathrm{rm}, j)}^{0}\left[v_{2}\right]=t_{2}^{2}\left(u_{1}^{2}+a_{j} u_{1}+b_{j}\right) .
\end{aligned}
$$

Since $f_{1}, f_{3} \in \mathcal{O}_{v}$ and $w_{(\mathrm{rm}, j)}^{0}$ satisfies Condition 5.10, $t_{1}, t_{2} u_{1}, t_{2} \in \mathcal{O}_{v}$. Since $\chi(g)=t_{1}^{2} t_{2}^{2} \in \mathcal{O}_{v}^{\times}, t_{1}, t_{2} \in \mathcal{O}_{v}^{\times}$. Thus, $g_{1} \in K_{2, v}$. 
We next prove that $w_{(\mathrm{rm}, j)}^{0}$ satisfies Condition 5.7. Suppose that $g w_{(\mathrm{rm}, j)}^{0} \in$ $V_{\mathcal{O}_{v}}$. By multiplying an element of $K_{2, v}$ from the left and an element of $\widetilde{T}_{k_{v}} \subseteq$ $G_{w_{(\mathrm{rm}, j)}^{0}} k_{v}$ from the right, we may assume that $g_{1}$ is in the form (5.16) and $t_{1}=1$. Then

$$
\begin{aligned}
& f_{1}=t_{0} w_{(\mathrm{rm}, j)}^{0}\left[v_{1}\right]=t_{0}, \\
& f_{3}=t_{0} w_{(\mathrm{rm}, j)}^{0}\left[v_{2}\right]=t_{0} t_{2}^{2}\left(u_{1}^{2}+a_{j} u_{1}+b_{j}\right) .
\end{aligned}
$$

By assumption, $f_{1}=t_{0} \in \mathcal{O}_{v}$. So $t_{0} f_{3}=w_{(\mathrm{rm}, j)}^{0}\left[t_{0} v_{2}\right] \in \mathcal{O}_{v}$. Since $w_{(\mathrm{rm}, j)}^{0}$ satisfies Condition 5.10, $t_{0} v_{2} \in \mathcal{O}_{v}^{2}$. Thus, $t_{0} t_{2} \in \mathcal{O}_{v}$. Since $\chi(g)=t_{0}^{2} t_{2}^{2}$, $\chi(g) \in \mathcal{O}_{v}$.

We next prove that $w_{(\mathrm{rm}, j)}^{0}$ satisfies Condition 5.8. Suppose that $g_{1}$ is as above, $g w_{(\mathrm{rm}, j)}^{0} \in V_{\mathcal{O}_{v}}$ and $\chi(g)=\left(t_{0} t_{2}\right)^{2} \in \mathcal{O}_{v}^{\times}$. Then $t_{0} t_{2} \in \mathcal{O}_{v}^{\times}$. By assumption, $t_{0} \in \mathcal{O}_{v}$.

We first assume that $\operatorname{ord}_{v}\left(t_{2}\right) \geq 0$. Since $t_{0} \in \mathcal{O}_{v}$ and $\operatorname{ord}_{v}\left(t_{0} t_{2}\right)=0$, $t_{0}, t_{2} \in \mathcal{O}_{v}^{\times}$. Since

$$
\operatorname{ord}_{v}\left(f_{3}\right)=\operatorname{ord}_{v}\left(u_{1}^{2}+a_{j} u_{1}+b_{j}\right) \geq 0,
$$

$u_{1} \in \mathcal{O}_{v}$. Thus, $g \in K_{v} \subseteq K_{v} G_{w_{(\mathrm{rm}, j)}^{0} k_{v}}$.

We next assume that $\operatorname{ord}_{v}\left(t_{2}\right)<0$. Since $\operatorname{ord}_{v}\left(t_{0} t_{2}\right)=0, \operatorname{ord}_{v}\left(t_{0} t_{2}^{2}\right)<0$. Since $f_{3}=t_{0} t_{2}^{2}\left(u_{1}^{2}+a_{j} u_{1}+b_{j}\right) \in \mathcal{O}_{v}$,

$$
\operatorname{ord}_{v}\left(u_{1}^{2}+a_{j} u_{1}+b_{j}\right)>0 .
$$

Thus, $\operatorname{ord}_{v}\left(u_{1}\right) \geq 1$ and so $\operatorname{ord}_{v}\left(u_{1}^{2}+a_{j} u_{1}+b_{j}\right)=1$. Since

$$
\operatorname{ord}_{v}\left(f_{3}\right)=\operatorname{ord}_{v}\left(t_{0} t_{2}^{2}\left(u_{1}^{2}+a_{j} u_{1}+b_{j}\right)\right)=1+\operatorname{ord}_{v}\left(t_{2}\right)
$$

and $f_{3} \in \mathcal{O}_{v}, \operatorname{ord}_{v}\left(t_{2}\right)=-1$ and so $\operatorname{ord}_{v}\left(t_{0}\right)=1$.

It is easy to see that

$$
\left(b_{j}^{-1},\left(\begin{array}{cc}
1 & 0 \\
0 & b_{j}
\end{array}\right)\left(\begin{array}{ll}
0 & 1 \\
1 & 0
\end{array}\right)\right) \in G_{w_{(\mathrm{rm}, j)}^{0} k_{v}}
$$

and

$$
g\left(b_{j}^{-1},\left(\begin{array}{ll}
1 & 0 \\
0 & b_{j}
\end{array}\right)\left(\begin{array}{ll}
0 & 1 \\
1 & 0
\end{array}\right)\right)=\left(t_{0} b_{j}^{-1},\left(\begin{array}{cc}
1 & 0 \\
t_{2} u_{1} & t_{2} b_{j}
\end{array}\right)\left(\begin{array}{ll}
0 & 1 \\
1 & 0
\end{array}\right)\right) \in K_{v} .
$$

Thus, $g \in K_{v} G_{w_{(\mathrm{rm}, j)}^{0} k_{v}}$. 
Let $n=4$. We first prove that $w_{\mathrm{dq}}^{0}$ satisfies Condition 5.10. Suppose that $v=\left(u_{1}, u_{2}, u_{3}, u_{4}\right) \in k_{v}^{4}$ and

$$
w_{\mathrm{dq}}^{0}[v]=\left(u_{1}^{2}+a_{0} u_{1} u_{2}+b_{0} u_{2}^{2}\right)+\pi_{v}\left(u_{3}^{2}+a_{0} u_{3} u_{4}+b_{0} u_{4}^{2}\right) \in \mathcal{O}_{v} .
$$

Since $\operatorname{ord}_{v}\left(u_{1}^{2}+a_{0} u_{1} u_{2}+b_{0} u_{2}^{2}\right)$ is even and $\operatorname{ord}_{v}\left(\pi_{v}\left(u_{3}^{2}+a_{0} u_{3} u_{4}+b_{0} u_{4}^{2}\right)\right)$ is odd for any $u_{1}, u_{2}, u_{3}, u_{4} \in k_{v}, u_{1}^{2}+a_{0} u_{1} u_{2}+b_{0} u_{2}^{2}, \pi_{v}\left(u_{3}^{2}+a_{0} u_{3} u_{4}+b_{0} u_{4}^{2}\right) \in$ $\mathcal{O}_{v}$. Thus, $u_{1}, u_{2}, u_{3}, u_{4} \in \mathcal{O}_{v}$.

We now prove that $w_{\mathrm{dq}}^{0}$ satisfies Conditions 5.7, 5.8 and 5.9. For four row vectors $v_{1}, v_{2}, v_{3}, v_{4} \in k_{v}^{4}$ and $t_{0} \in k_{v}^{\times}$, we put

$$
g_{1}=\left(\begin{array}{l}
v_{1} \\
v_{2} \\
v_{3} \\
v_{4}
\end{array}\right), \quad g=\left(t_{0}, g_{1}\right), \quad g w_{\mathrm{dq}}^{0}=\left(\begin{array}{cccc}
2 f_{1} & f_{2} & f_{3} & f_{4} \\
f_{2} & 2 f_{5} & f_{6} & f_{7} \\
f_{3} & f_{6} & 2 f_{8} & f_{9} \\
f_{4} & f_{7} & f_{9} & 2 f_{10}
\end{array}\right) .
$$

It is easy to see that

$$
f_{1}=t_{0} w_{\mathrm{dq}}^{0}\left[v_{1}\right], \quad f_{5}=t_{0} w_{\mathrm{dq}}^{0}\left[v_{2}\right], \quad f_{8}=t_{0} w_{\mathrm{dq}}^{0}\left[v_{3}\right], \quad f_{10}=t_{0} w_{\mathrm{dq}}^{0}\left[v_{4}\right] .
$$

We first prove that $w_{\mathrm{dq}}^{0}$ satisfies Condition 5.9. Suppose that $t_{0}=1$, $g w_{\mathrm{dq}}^{0} \in V_{\mathcal{O}_{v}}$ and $\chi(g) \in \mathcal{O}_{v}^{\times}$. Multiplying an element of $K_{4, v}$ from the left, we may assume that $g_{1}$ is in the following form:

$$
\left(\begin{array}{cccc}
t_{1} & 0 & 0 & 0 \\
0 & t_{2} & 0 & 0 \\
0 & 0 & t_{3} & 0 \\
0 & 0 & 0 & t_{4}
\end{array}\right)\left(\begin{array}{cccc}
1 & 0 & 0 & 0 \\
u_{1} & 1 & 0 & 0 \\
u_{2} & u_{3} & 1 & 0 \\
u_{4} & u_{5} & u_{6} & 1
\end{array}\right)=\left(\begin{array}{cccc}
t_{1} & 0 & 0 & 0 \\
t_{2} u_{1} & t_{2} & 0 & 0 \\
t_{3} u_{2} t_{3} u_{3} & t_{3} & 0 \\
t_{4} u_{4} & t_{4} u_{5} & t_{4} u_{6} & t_{4}
\end{array}\right)
$$

where $t_{1}, \cdots, t_{4} \in k_{v}^{\times}$and $u_{1}, \cdots, u_{6} \in k_{v}$. Let $f_{1}, f_{5}, f_{8}, f_{10}$ be as above.

Then

$$
\begin{aligned}
& f_{1}=w_{\mathrm{dq}}^{0}\left[v_{1}\right]=t_{1}^{2}, \\
& f_{5}=w_{\mathrm{dq}}^{0}\left[v_{2}\right]=t_{2}^{2}\left(u_{1}^{2}+a_{0} u_{1}+b_{0}\right), \\
& f_{8}=w_{\mathrm{dq}}^{0}\left[v_{3}\right]=t_{3}^{2}\left(u_{2}^{2}+a_{0} u_{2} u_{3}+b_{0} u_{3}^{2}+\pi_{v}\right), \\
& f_{10}=w_{\mathrm{dq}}^{0}\left[v_{4}\right]=t_{4}^{2}\left(u_{4}^{2}+a_{0} u_{4} u_{5}+b_{0} u_{5}^{2}+\pi_{v} u_{6}^{2}+a_{0} \pi_{v} u_{6}+b_{0} \pi_{v}\right) .
\end{aligned}
$$

Since $f_{1}, f_{5}, f_{8}, f_{10} \in \mathcal{O}_{v}$ and $w_{\mathrm{dq}}^{0}$ satisfies Condition 5.10,

$$
t_{1}, t_{2} u_{1}, t_{2}, t_{3} u_{2}, t_{3} u_{3}, t_{3}, t_{4} u_{4}, t_{4} u_{5}, t_{4} u_{6}, t_{4} \in \mathcal{O}_{v}
$$

Since $\chi(g)=t_{1}^{2} t_{2}^{2} t_{3}^{2} t_{4}^{2} \in \mathcal{O}_{v}^{\times}, t_{1}, t_{2}, t_{3}, t_{4} \in \mathcal{O}_{v}^{\times}$. Thus, $g_{1} \in K_{4, v}$. 
We next prove that $w_{\mathrm{dq}}^{0}$ satisfies Condition 5.7. Suppose that $g w_{\mathrm{dq}}^{0} \in V_{\mathcal{O}_{v}}$. By multiplying an element of $K_{4, v}$ from the left and an element of $\widetilde{T}_{k_{v}} \subseteq G_{w_{\mathrm{dq}}^{0} k_{v}}$ from the right, we may assume that $g_{1}$ is in the form (5.19) and $t_{1}=1$. Then

$$
\begin{aligned}
& f_{1}=t_{0} w_{\mathrm{dq}}^{0}\left[v_{1}\right]=t_{0}, \\
& f_{5}=t_{0} w_{\mathrm{dq}}^{0}\left[v_{2}\right]=t_{0} t_{2}^{2}\left(u_{1}^{2}+a_{0} u_{1}+b_{0}\right), \\
& f_{8}=t_{0} w_{\mathrm{dq}}^{0}\left[v_{3}\right]=t_{0} t_{3}^{2}\left(u_{2}^{2}+a_{0} u_{2} u_{3}+b_{0} u_{3}^{2}+\pi_{v}\right), \\
& f_{10}=t_{0} w_{\mathrm{dq}}^{0}\left[v_{4}\right]=t_{0} t_{4}^{2}\left(u_{4}^{2}+a_{0} u_{4} u_{5}+b_{0} u_{5}^{2}+\pi_{v} u_{6}^{2}+a_{0} \pi_{v} u_{6}+b_{0} \pi_{v}\right) .
\end{aligned}
$$

Since $f_{1} \in \mathcal{O}_{v}, t_{0} \in \mathcal{O}_{v}$. So $t_{0} f_{4}=w_{\mathrm{dq}}^{0}\left[t_{0} v_{2}\right], t_{0} f_{8}=w_{\mathrm{dq}}^{0}\left[t_{0} v_{3}\right], t_{0} f_{10}=$ $w_{\mathrm{dq}}^{0}\left[t_{0} v_{4}\right] \in \mathcal{O}_{v}$. Since $w_{\mathrm{dq}}^{0}$ satisfies Condition 5.10, $t_{0} v_{2}, t_{0} v_{3}, t_{0} v_{4} \in \mathcal{O}_{v}^{3}$. Thus, $t_{0} t_{2}, t_{0} t_{3}, t_{0} t_{4} \in \mathcal{O}_{v}$

We consider $f_{5}$. Since $f_{5}=t_{0} t_{2}^{2}\left(u_{1}^{2}+a_{0} u_{1}+b_{0}\right) \in \mathcal{O}_{v}$ and $\operatorname{ord}_{v}\left(u_{1}^{2}+a_{0} u_{1}+\right.$ $\left.b_{0}\right) \leq 0, \operatorname{ord}_{v}\left(t_{0} t_{2}^{2}\right) \geq 0$.

We consider $f_{8}$. Since $\operatorname{ord}_{v}\left(\pi_{v}\right)=1$ and $\operatorname{ord}_{v}\left(u_{2}^{2}+a_{0} u_{2} u_{3}+b_{0} u_{3}^{2}\right)$ is even for any $u_{2}, u_{3} \in k_{v}, t_{0} t_{3}^{2} \pi_{v} \in \mathcal{O}_{v}$ and so $\operatorname{ord}_{v}\left(t_{0} t_{3}^{2}\right) \geq-1$.

We consider $f_{10}$. Since $\operatorname{ord}_{v}\left(u_{4}^{2}+a_{0} u_{4} u_{5}+b_{0} u_{5}^{2}\right)$ is even and $\operatorname{ord}_{v}\left(\pi_{v}\left(u_{6}^{2}+\right.\right.$

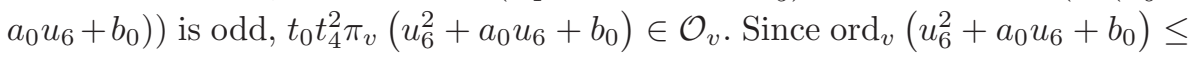
$0, \operatorname{ord}_{v}\left(t_{0} t_{4}^{2}\right) \geq-1$.

Suppose that $\operatorname{ord}(\chi(g))<0$. Since

$$
\operatorname{ord}_{v}\left(t_{0}^{2} t_{2}^{2}\right) \geq 0, \operatorname{ord}_{v}\left(t_{0} t_{3}^{2}\right), \operatorname{ord}_{v}\left(t_{0} t_{4}^{2}\right) \geq-1,
$$

$\operatorname{ord}(\chi(g))=\operatorname{ord}\left(t_{0}^{4} t_{2}^{2} t_{3}^{2} t_{4}^{2}\right) \geq-2$. Since ord $(\chi(g))$ is even, $\operatorname{ord}(\chi(g))=-2$ and

$$
\operatorname{ord}_{v}\left(t_{0}^{2} t_{2}^{2}\right)=0, \operatorname{ord}_{v}\left(t_{0} t_{3}^{2}\right)=\operatorname{ord}_{v}\left(t_{0} t_{4}^{2}\right)=-1 .
$$

Since $t_{0} t_{3} \in \mathcal{O}_{v}, \operatorname{ord}_{v}\left(t_{3}\right) \leq-1$ and $\operatorname{ord}_{v}\left(t_{0}\right) \geq 1$. Since $\operatorname{ord}_{v}\left(t_{0} t_{2}\right)=0$, $\operatorname{ord}_{v}\left(t_{2}\right)=-\operatorname{ord}_{v}\left(t_{0}\right) \leq-1$. This contradicts to the condition $\operatorname{ord}_{v}\left(t_{0} t_{2}^{2}\right) \geq 0$. Therefore, $\operatorname{ord}(\chi(g)) \geq 0$.

We next prove that $w_{\mathrm{dq}}^{0}$ satisfies Condition 5.8. Suppose that $g=\left(t_{0}, g_{1}\right) \in$ $G_{k_{v}}$ where $g_{1}$ is as above, $g w_{\mathrm{dq}}^{0} \in V_{\mathcal{O}_{v}}$ and $\chi(g) \in \mathcal{O}_{v}^{\times}$. By multiplying an element of $K_{4, v}$ from the left and an element of $\widetilde{T}_{k_{v}} \subseteq G_{w_{\mathrm{dq}}^{0} k_{v}}$ from the right, we may assume that $g_{1}$ is in the form (5.19) and $t_{1}=1$. Then $f_{1}, f_{5}, f_{8}, f_{10}$ are as in (5.20). So

$$
\operatorname{ord}_{v}\left(t_{0}\right), \operatorname{ord}_{v}\left(t_{0} t_{2}^{2}\right) \geq 0, \operatorname{ord}_{v}\left(t_{0} t_{3}^{2}\right), \operatorname{ord}_{v}\left(t_{0} t_{4}^{2}\right) \geq-1
$$

and

$$
\begin{aligned}
& t_{0} v_{2}=\left(t_{0} t_{2} u_{1}, t_{0} t_{2}, 0,0\right), \\
& t_{0} v_{3}=\left(t_{0} t_{3} u_{2}, t_{0} t_{3} u_{3}, t_{0} t_{3}, 0\right), \\
& t_{0} v_{4}=\left(t_{0} t_{4} u_{4}, t_{0} t_{4} u_{5}, t_{0} t_{4} u_{6}, t_{0} t_{4}\right)
\end{aligned}
$$


are elements of $\mathcal{O}_{v}^{4}$. Since

$$
\begin{gathered}
\operatorname{ord}_{v}(\chi(g))=\operatorname{ord}_{v}\left(t_{0}^{2} t_{2}^{2}\right)+\operatorname{ord}_{v}\left(t_{0} t_{3}^{2}\right)+\operatorname{ord}_{v}\left(t_{0} t_{4}^{2}\right)=0, \\
\left(\operatorname{ord}_{v}\left(t_{0}^{2} t_{2}^{2}\right), \operatorname{ord}_{v}\left(t_{0} t_{3}^{2}\right), \operatorname{ord}_{v}\left(t_{0} t_{4}^{2}\right)\right)
\end{gathered}
$$

is equal to $(2,-1,-1),(0,-1,1),(0,0,0)$ or $(0,1,-1)$.

Suppose that $\operatorname{ord}_{v}\left(t_{0} t_{2}\right)=0$. If $\operatorname{ord}_{v}\left(t_{0} t_{3}^{2}\right)=-1\left(\operatorname{resp} . \operatorname{ord}_{v}\left(t_{0} t_{4}^{2}\right)=-1\right)$, $\operatorname{ord}_{v}\left(t_{3}\right) \leq-1\left(\operatorname{resp} . \operatorname{ord}_{v}\left(t_{4}\right) \leq-1\right)$ and $\operatorname{ord}_{v}\left(t_{0}\right) \geq 1$. Since $\operatorname{ord}_{v}\left(t_{0} t_{2}\right)=0$, $\operatorname{ord}_{v}\left(t_{0} t_{2}^{2}\right) \leq-1$. This contradicts to the condition $\operatorname{ord}_{v}\left(t_{0} t_{2}^{2}\right) \in \mathcal{O}_{v}$. So (5.21) is equal to $(2,-1,-1)$ or $(0,0,0)$.

Suppose that (5.21) is equal to $(0,0,0)$. Since $t_{0}, t_{0} t_{2}^{2} \in \mathcal{O}_{v}$ and $\operatorname{ord}_{v}\left(t_{0}^{2} t_{2}^{2}\right)$ $=0, t_{0}, t_{2} \in \mathcal{O}_{v}^{\times}$. Since $t_{0} t_{3}^{2}, t_{0} t_{4}^{2} \in \mathcal{O}_{v}^{\times}, t_{3}, t_{4} \in \mathcal{O}_{v}^{\times}$. Since $t_{0} v_{2}, t_{0} v_{3}, t_{0} v_{4} \in$ $\mathcal{O}_{v}^{4}, u_{i} \in \mathcal{O}_{v}$ for all $1 \leq i \leq 6$. Thus, $g \in K_{v} \subseteq K_{v} G_{w_{\mathrm{dq}}^{0} k_{v}}$.

Suppose that (5.21) is equal to $(2,-1,-1)$. Since $\operatorname{ord}_{v}\left(t_{0} t_{3}^{2}\right)=-1$, $\operatorname{ord}_{v}\left(t_{0}\right)$ is odd and so $\operatorname{ord}_{v}\left(t_{0}\right) \geq 1$. Since $\operatorname{ord}_{v}\left(t_{0}\right)+\operatorname{ord}_{v}\left(t_{0} t_{2}^{2}\right)=2$ and $t_{0} t_{2}^{2} \in \mathcal{O}_{v}, \operatorname{ord}_{v}\left(t_{0}\right)=1$ and $\operatorname{ord}_{v}\left(t_{0} t_{2}^{2}\right)=1$. Thus,

$$
\left(\operatorname{ord}_{v}\left(t_{0}\right), \operatorname{ord}_{v}\left(t_{2}\right), \operatorname{ord}_{v}\left(t_{3}\right), \operatorname{ord}_{v}\left(t_{4}\right)\right)=(1,0,-1,-1) .
$$

We consider $f_{5}=t_{0} t_{2}^{2}\left(u_{1}^{2}+a_{0} u_{1}+b_{0}\right)$. Since $\operatorname{ord}_{v}\left(t_{0} t_{2}^{2}\right)=1$ and $\operatorname{ord}_{v}\left(u_{1}^{2}+\right.$ $\left.a_{0} u_{1}+b_{0}\right)$ is even for any $u_{1} \in k_{v}, \operatorname{ord}_{v}\left(u_{1}^{2}+a_{0} u_{1}+b_{0}\right) \geq 0$. This implies that $u_{1} \in \mathcal{O}_{v}$.

We consider $f_{8}=t_{0} t_{3}^{2}\left(u_{2}^{2}+a_{0} u_{2} u_{3}+b_{0} u_{3}^{2}+\pi_{v}\right)$. Since $\operatorname{ord}_{v}\left(u_{2}^{2}+a_{0} u_{2} u_{3}+\right.$ $\left.b_{0} u_{3}^{2}\right)$ is even for any $u_{2}, u_{3} \in k_{v}$ and $\operatorname{ord}_{v}\left(\pi_{v}\right)=1, t_{0} t_{3}^{2}\left(u_{2}^{2}+a_{0} u_{2} u_{3}+\right.$ $\left.b_{0} u_{3}^{2}\right) \in \mathcal{O}_{v}$. Since $\operatorname{ord}_{v}\left(t_{0} t_{3}^{2}\right)=-1, \operatorname{ord}_{v}\left(u_{2}^{2}+a_{0} u_{2} u_{3}+b_{0} u_{3}^{2}\right) \geq 2$. Thus, $\operatorname{ord}_{v}\left(u_{2}\right), \operatorname{ord}_{v}\left(u_{3}\right) \geq 1$. Since $f_{10} \in \mathcal{O}_{v}$ and $\operatorname{ord}_{v}\left(t_{0} t_{4}^{2}\right)=-1, \operatorname{ord}_{v}\left(u_{4}\right), \operatorname{ord}_{v}\left(u_{5}\right)$ $\geq 1$ and $\operatorname{ord}_{v}\left(u_{6}\right) \geq 0$.

It is easy to see that

$$
\left(\pi_{v}^{-1},\left(\begin{array}{cc}
I_{2} & \\
& \pi_{v} I_{2}
\end{array}\right)\left(\begin{array}{c}
I_{2} \\
I_{2}
\end{array}\right)\right) \in G_{w_{\mathrm{dq}}^{0} k_{v}}
$$

and

$$
\begin{aligned}
& \left(t_{0},\left(\begin{array}{cccc}
1 & 0 & 0 & 0 \\
t_{2} u_{1} & t_{2} & 0 & 0 \\
t_{3} u_{2} t_{3} u_{3} & t_{3} & 0 \\
t_{4} u_{4} t_{4} u_{5} t_{4} u_{6} & t_{4}
\end{array}\right)\right)\left(\pi_{v}^{-1},\left(\begin{array}{ll}
I_{2} & \\
& \pi_{v} I_{2}
\end{array}\right)\left(\begin{array}{cc}
I_{2} \\
I_{2}
\end{array}\right)\right) \\
& =\left(t_{0} \pi_{v}^{-1},\left(\begin{array}{cccc}
0 & 0 & 1 & 0 \\
0 & 0 & t_{2} u_{1} & t_{2} \\
t_{3} \pi_{v} & 0 & t_{3} u_{2} t_{3} u_{3} \\
t_{4} \pi_{v} u_{6} & t_{4} \pi_{v} t_{4} u_{4} t_{4} u_{5}
\end{array}\right)\right) \in K_{v} .
\end{aligned}
$$


By (5.22) and the informations on $\operatorname{ord}_{v}\left(u_{1}\right), \cdots, \operatorname{ord}_{v}\left(u_{6}\right)$ obtained above, the condition $g \in K_{v} G_{w_{\mathrm{dq}}^{0} k_{v}}$ follows. This completes the proof of Lemma 5.14.

In the following, we consider the discriminants of representatives. If $w_{v \text {,i }}$ is a standard representative, we use the letter $m$ for the Witt index as before. Also $m_{0}=n-2 m$. So if $n \geq 3$ is odd then $m_{0}=1,3$ according as $\dot{\mathrm{i}}=\mathrm{sp}, \mathrm{rm}$. If $n \geq 4$ is even then $m_{0}=0,2,2,4$ according as $\dot{i}=s p$, in, rm, dq. We use the same $m, m_{0}$ for alternative representatives also.

Proposition 5.23. If $n \geq 3$ is odd, $w_{\mathrm{sp}}$ and $w_{\mathrm{rm}}$ satisfy Condition 5.7. Moreover,

$$
\Delta_{w_{\mathrm{sp}}, v}=1, \quad \Delta_{w_{\mathrm{rm}}, v}=q_{v} .
$$

If $n \geq 2$ is even, $w_{\mathrm{sp}}, w_{\mathrm{in}}, w_{(\mathrm{rm}, j)}$ for $1 \leq j \leq \lambda_{v}$ and $w_{\mathrm{dq}}$ (if $n \geq 4$ ) satisfy Conditions 5.7 and 5.8. Moreover,

$$
\Delta_{w_{\mathrm{sp}}, v}=\Delta_{w_{\mathrm{in}}, v}=1, \Delta_{w_{(\mathrm{rm}, j)}, v}=q_{v}^{\operatorname{ord}_{v}\left(\Delta_{F_{j} / k_{v}}\right)}\left(1 \leq j \leq \lambda_{v}\right), \Delta_{w_{\mathrm{dq}}, v}=q_{v}^{2} .
$$

Proof. It suffices to prove that the statement holds for $w_{\mathrm{i}}^{\prime}$ in (4.18) instead of $w_{\mathrm{i}}$.

We first prove that $w_{\dot{\mathrm{i}}}^{\prime}$ satisfies Condition 5.7. Let $g=\left(t_{0}, g_{1}\right) \in G_{k_{v}}$. Suppose that $g w_{\dot{\mathrm{i}}}^{\prime} \in V_{\mathcal{O}_{v}}$.

By multiplying an element of $K_{v}$ from the left and an element of $\widetilde{T}_{k_{v}} \subseteq$ $G_{w_{\mathrm{i}}^{\prime} k_{v}}$ from the right, $g_{1}$ can be made into the following form:

$$
g_{1}=\left(\begin{array}{ccc}
X_{11} & 0 & 0 \\
X_{21} & X_{22} & 0 \\
X_{31} & X_{32} & X_{33}
\end{array}\right)
$$

where $X_{11},{ }^{t} X_{33} \in B_{m k_{v}}, X_{21},{ }^{t} X_{32} \in \mathrm{M}\left(m_{0}, m\right)_{k_{v}}, X_{31} \in \mathrm{M}(m, m)_{k_{v}}$ and

$$
X_{22}=\left(\begin{array}{cccc}
1 & & & \\
& t_{2} & & \\
& & \ddots & \\
& & & t_{m_{0}}
\end{array}\right)\left(\begin{array}{ccc}
1 & & \\
& \ddots & \\
u & & 1
\end{array}\right) \in B_{m_{0} k_{v}}
$$

It is easy to see that $G_{w_{\mathrm{i}}^{\prime} k_{v}}$ contains the following matrices

$$
\left(\begin{array}{ccc}
{ }^{t} X_{33} & 0 & 0 \\
0 & I_{m_{0}} & 0 \\
0 & 0 & X_{33}^{-1}
\end{array}\right), \quad\left(\begin{array}{ccc}
I_{m} & 0 & 0 \\
w_{\dot{\mathrm{i}}}^{0 t} X_{32} & I_{m_{0}} & 0 \\
Y_{31} & X_{32} & I_{m}
\end{array}\right)
$$


where $Y_{31} \in B_{m k_{v}}$ and $Y_{31}+{ }^{t} Y_{31}=w_{\mathrm{i}}^{0}\left[X_{32}\right]$.

By multiplying elements in the above forms from the right, $g_{1}$ in (5.24) can be made into the following form:

$$
\left(\begin{array}{ccc}
X_{11} & 0 & 0 \\
X_{21} & X_{22} & 0 \\
X_{31} & 0 & I_{m}
\end{array}\right)
$$

where $X_{21} \in \mathrm{M}\left(m_{0}, m\right)_{k_{v}}, X_{31} \in \mathrm{M}(m, m)_{k_{v}}$ and

$$
\begin{aligned}
& X_{11}=\left(\begin{array}{ccc}
t_{1}^{\prime} & & \\
& \ddots & \\
& & t_{m}^{\prime}
\end{array}\right)\left(\begin{array}{ccc}
1 & & \\
& \ddots & \\
u^{\prime} & & 1
\end{array}\right) \in B_{m k_{v}}, \\
& X_{22}=\left(\begin{array}{llll}
1 & & & \\
& t_{2} & & \\
& & \ddots & \\
& & & t_{m_{0}}
\end{array}\right)\left(\begin{array}{lll}
1 & & \\
& \ddots & \\
u & & 1
\end{array}\right) \in B_{m_{0} k_{v}} .
\end{aligned}
$$

We express $g w_{\mathrm{i}}^{\prime}$ as follows:

$$
g w_{\mathrm{i}}^{\prime}=\left(\begin{array}{ccc}
F_{1} & F_{2} & F_{3} \\
{ }^{t} F_{2} & F_{4} & F_{5} \\
{ }^{t} F_{3} & F_{5} & F_{6}
\end{array}\right)
$$

where $F_{1}, F_{3}, F_{6} \in \mathrm{M}(m, m)_{k_{v}}, F_{2},{ }^{t} F_{5} \in \mathrm{M}\left(m, m_{0}\right)_{k_{v}}, F_{4} \in \mathrm{M}\left(m_{0}, m_{0}\right)_{k_{v}}$. Then

$$
\begin{aligned}
& F_{1}=0, \quad F_{2}=0, \\
& F_{3}=-t_{0} X_{11}, F_{4}=t_{0} w_{\mathrm{i}}^{0}\left[X_{22}\right], \\
& F_{5}=-t_{0} X_{21}, F_{6}=-t_{0}\left({ }^{t} X_{31}+X_{31}\right) .
\end{aligned}
$$

To consider $F_{4}$ corresponds to the situation where the Witt index is 0 and $g^{0}=\left(t_{0}, X_{22}\right)$. So Lemmas 5.11 and 5.14 imply that $t_{0}^{m_{0}}\left(t_{2} \cdots t_{m_{0}}\right)^{2} \in \mathcal{O}_{v}$. This is $\chi\left(g^{0}\right)$ for $m=0$.

We consider $F_{3}$. Since $F_{3}=-t_{0} X_{11} \in \mathrm{M}(m, m)_{\mathcal{O}_{v}}$, diagonal entries $t_{0} t_{1}^{\prime}, \cdots, t_{0} t_{m}^{\prime}$ of $t_{0} X_{11}$ are elements of $\mathcal{O}_{v}$. Therefore,

$$
\chi(g)=t_{0}^{m_{0}}\left(t_{2} \cdots t_{m_{0}}\right)^{2}\left(t_{0} t_{1}^{\prime}\right)^{2} \cdots\left(t_{0} t_{m}^{\prime}\right)^{2} \in \mathcal{O}_{v} .
$$

Thus, $w_{\mathrm{i}}^{\prime}$ satisfies Condition 5.7.

We next prove that $w_{\mathrm{i}}^{\prime}$ satisfies Condition 5.8 if $n \geq 4$ is even. As above, we assume that $g=\left(t_{0}, g_{1}\right)$ where $t_{0} \in k_{v}^{\times}$and $g_{1}$ is in the form (5.25) and that

$$
\chi(g)=t_{0}^{m_{0}}\left(t_{2} \cdots t_{m_{0}}\right)^{2}\left(t_{0} t_{1}^{\prime}\right)^{2} \cdots\left(t_{0} t_{m}^{\prime}\right)^{2} \in \mathcal{O}_{v}^{\times} .
$$


Since $t_{0}^{m_{0}}\left(t_{2} \cdots t_{m_{0}}\right)^{2}, t_{0} t_{1}^{\prime}, \ldots, t_{0} t_{m}^{\prime}$ are elements of $\mathcal{O}_{v}$

$$
t_{0}^{m_{0}}\left(t_{2} \cdots t_{m_{0}}\right)^{2}, t_{0} t_{1}^{\prime}, \cdots, t_{0} t_{m}^{\prime} \in \mathcal{O}_{v}^{\times} .
$$

By Lemma 5.14, there exists $\left(\kappa_{1}, \kappa_{2}\right) \in K_{1, v} \times K_{m_{0}, v}$ and $\left(h_{1}, h_{2}\right) \in(\mathrm{GL}(1) \times$ $\left.\operatorname{GL}\left(m_{0}\right)\right)_{w_{\mathrm{1}}^{0} k_{v}}$ such that $\left(t_{0}, X_{22}\right)=\left(\kappa_{1}, \kappa_{2}\right)\left(h_{1}, h_{2}\right)$. So, by multiplying

$$
\left(\kappa_{1},\left(\begin{array}{ccc}
I_{m} & 0 & 0 \\
0 & \kappa_{2} & 0 \\
0 & 0 & I_{m}
\end{array}\right)\right)^{-1} \in K_{v}, \quad\left(h_{1},\left(\begin{array}{ccc}
h_{1}^{-1} I_{m} & 0 & 0 \\
0 & h_{2} & 0 \\
0 & 0 & I_{m}
\end{array}\right)\right)^{-1} \in G_{w_{\mathrm{i}}^{\prime} k_{v}}
$$

from the left and the right, we may assume that

$$
\left(t_{0}, g_{1}\right)=\left(1,\left(\begin{array}{ccc}
X_{11} & 0 & 0 \\
X_{21} & I_{m_{0}} & 0 \\
X_{31} & 0 & I_{m}
\end{array}\right)\right)
$$

We express $g w_{\mathrm{i}}^{\prime}$ as $(5.26)$. Since $t_{0}=1, F_{3}=-X_{11} \in \mathrm{M}(m, m)_{\mathcal{O}_{v}}$ and $F_{5}=-X_{21} \in \mathrm{M}\left(m_{0}, m\right)_{\mathcal{O}_{v}}, X_{11} \in \mathrm{GL}(m)_{\mathcal{O}_{v}}$ and $X_{21} \in \mathrm{M}\left(m_{0}, m\right)_{\mathcal{O}_{v}}$. Note that $G_{w_{\mathrm{i}}^{\prime}} k_{v}$ contains matrices in the following form:

$$
\left(\begin{array}{ccc}
I_{m} & 0 & 0 \\
0 & I_{m_{0}} & 0 \\
Y_{31} & 0 & I_{m}
\end{array}\right)
$$

where $Y_{31} \in \mathrm{M}(m, m)_{k_{v}}$ and $Y_{31}+{ }^{t} Y_{31}=0$.

Multiplying an element in the above form from the right, we may assume that $X_{31}$ is in the following form:

$$
X_{31}=\left(\begin{array}{ccc}
u_{11}^{\prime} & & 0 \\
\vdots & \ddots & \\
u_{m 1}^{\prime} & \cdots & u_{m m}^{\prime}
\end{array}\right) .
$$

Since $g w_{\mathrm{i}}^{\prime} \in V_{\mathcal{O}_{v}}$ and

$$
-F_{6}={ }^{t} X_{31}+X_{31}=\left(\begin{array}{ccc}
2 u_{11}^{\prime} & \cdots & u_{m 1}^{\prime} \\
\vdots & \ddots & \vdots \\
u_{m 1}^{\prime} & \cdots & 2 u_{m m}^{\prime}
\end{array}\right)
$$

$u_{i j}^{\prime} \in \mathcal{O}_{v}$ and so $X_{31} \in \mathrm{M}(m, m)_{\mathcal{O}_{v}}$. Thus, $g \in K_{v} G_{w_{\mathrm{i}}^{\prime} k_{v}}$.

This completes the proof of Proposition 5.23. 
Corollary 5.28. If $x \in V_{k}^{\text {ss }}$ then there exists a finite subset $\mathfrak{M}_{\infty} \cup$ $\mathfrak{M}_{\text {dy }} \subseteq S \subseteq \mathfrak{M}$ such that $x$ is unramified over $k_{v}$ if $v \notin S$.

Proof. We choose $S$ so that if $v \notin S$ then $x \in V_{\mathcal{O}_{v}}$ and $|P(x)|_{v}=1$. By the definition of the discriminant, $\Delta_{x, v}=1$. Then the corollary follows from Proposition 5.23.

\section{$\S 6 . \quad$ Existence of Global Orbits with Prescribed Local Conditions}

In this section we assume that $n \geq 3$ is odd. The main purpose of this section is to describe the image of the natural map from $G_{k} \backslash V_{k}^{\text {ss }}$ to $\prod_{v \in \mathfrak{M}} G_{k_{v}} \backslash V_{k_{v}}^{\text {ss }}$ by the modified Hasse symbol.

We first review some facts concerning the action of $\mathrm{GL}(n)$ on quadratic forms. For $v \in \mathfrak{M}$ and $x_{v} \in V_{k_{v}}^{\text {ss }}$, we denote the classical discriminant of $x_{v}$ by $d_{v}\left(x_{v}\right)$. We denote the Hasse symbol of $x_{v}$ by $S_{v}\left(x_{v}\right)$ and the modified Hasse symbol $S_{v}\left(\left(\operatorname{det} M_{x_{v}}\right) x_{v}\right)$ by $\widetilde{S}_{v}\left(x_{v}\right)$ as in $\S 4$.

The following theorem is the Hasse-Minkowski Theorem (66:4) [22, p. 189].

Theorem 6.1. Let $x, y \in V_{k}^{\mathrm{ss}}$. Then $\mathrm{GL}(n)_{k} x=\mathrm{GL}(n)_{k} y$ if and only if $\mathrm{GL}(n)_{k_{v}} x=\mathrm{GL}(n)_{k_{v}}$ y for all $v \in \mathfrak{M}$.

In this paper we call the above theorem the Hasse principle for quadratic forms.

The following theorem is Theorem (72:1) [22, p. 203].

Theorem 6.2. $\quad$ Let $\left(x_{v}\right) \in \prod_{v \in \mathfrak{M}} V_{k_{v}}^{\mathrm{ss}}$. There exists $x \in V_{k}^{\mathrm{ss}}$ such that $\mathrm{GL}(n)_{k_{v}} x=\mathrm{GL}(n)_{k_{v}} x_{v}$ for all $v \in \mathfrak{M}$ if and only if $\left(x_{v}\right)$ satisfies the following three conditions.

(1) There exists $\alpha \in k$ such that $d_{v}\left(x_{v}\right) \equiv \alpha \bmod \left(k_{v}^{\times}\right)^{2}$ for all $v \in \mathfrak{M}$.

(2) $S_{v}\left(x_{v}\right)=1$ for all but finitely many $v \in \mathfrak{M}$.

(3) $\prod_{v \in \mathfrak{M}} S_{v}\left(x_{v}\right)=1$.

Definition 6.3. $\quad$ Let $\Phi$ be a map defined by

$$
G_{k} \backslash V_{k}^{\mathrm{ss}} \ni x \mapsto(x)_{v} \in \prod_{v \in \mathfrak{M}} G_{k_{v}} \backslash V_{k_{v}}^{\mathrm{ss}} .
$$

It is easy to see that $\Phi$ is well-defined.

Lemma 6.5. If $n \geq 3$ is odd, $\Phi$ is injective. 
Proof. Suppose that $x, x^{\prime} \in V_{k}^{\text {ss }}$ and that there exists $g_{v}=\left(t_{0, v}, g_{1, v}\right) \in$ $G_{k_{v}}$ such that $x^{\prime}=g_{v} x$ for all $v \in \mathfrak{M}$. We put $g_{0}=\operatorname{det} M_{x^{\prime}} / \operatorname{det} M_{x} \in k^{\times}$. Since $\chi\left(g_{v}\right) \equiv t_{0, v} \quad \bmod \left(k_{v}^{\times}\right)^{2}$,

$$
\operatorname{det} M_{x^{\prime}} \equiv \chi\left(g_{v}\right) \operatorname{det} M_{x} \equiv t_{0, v} \operatorname{det} M_{x} \bmod \left(k_{v}^{\times}\right)^{2} .
$$

So $g_{0} \equiv t_{0, v} \bmod \left(k_{v}^{\times}\right)^{2}$, which implies that

$$
\mathrm{GL}(n)_{k_{v}}\left(g_{0} x\right)=\mathrm{GL}(n)_{k_{v}}\left(t_{0, v} x\right)
$$

for all $v$. So

$$
\mathrm{GL}(n)_{k_{v}}\left(g_{0} x\right)=\mathrm{GL}(n)_{k_{v}}\left(t_{0, v} x\right)=\mathrm{GL}(n)_{k_{v}} x^{\prime}
$$

for all $v$. By Theorem 6.1, $\operatorname{GL}(n)_{k}\left(g_{0} x\right)=\mathrm{GL}(n)_{k} x^{\prime}$. Thus, $G_{k} x=G_{k} x^{\prime}$.

Proposition 6.6. $\quad$ By the map $\Phi$, the orbit space $G_{k} \backslash V_{k}^{\text {ss }}$ corresponds bijectively to the set of elements $\left(x_{v}\right) \in \prod_{v \in \mathfrak{M}} G_{k_{v}} \backslash V_{k_{v}}^{\text {ss }}$ which satisfy the following two conditions.

(1) $\widetilde{S}_{v}\left(x_{v}\right)=1$ for all but finitely many $v \in \mathfrak{M}$.

(2) $\prod_{v \in \mathfrak{M}} \widetilde{S}_{v}(x)=1$.

Proof. If $x \in V_{k}^{\text {ss }}$, then $\left(\left(\operatorname{det} M_{x}\right) x\right)_{v}$ clearly satisfies the above two conditions.

Suppose that $\left(x_{v}\right)_{v} \in \prod_{v \in \mathfrak{M}} G_{k_{v}} \backslash V_{k_{v}}^{\text {ss }}$ satisfies the above two conditions. Then $\left(\left(\operatorname{det} M_{x_{v}}\right) x_{v}\right)_{v} \in \prod_{v \in \mathfrak{M}} G_{k_{v}} \backslash V_{k_{v}}^{\mathrm{ss}}$ satisfies Conditions (2) and (3) in Theorem 6.2. Since $n$ is odd,

$$
d_{v}\left(\left(\operatorname{det} M_{x_{v}}\right) x_{v}\right) \equiv\left(\operatorname{det} M_{x_{v}}\right)^{n} d_{v}\left(x_{v}\right) \equiv\left(\operatorname{det} M_{x_{v}}\right)^{n+1} \equiv 1 \quad \bmod \left(k_{v}^{\times}\right)^{2}
$$

and that $1 \in k^{\times}$. So $\left(\left(\operatorname{det} M_{x_{v}}\right) x_{v}\right)_{v}$ also satisfies Conditions (1) in Theorem 6.2. By Theorem 6.2, there exists $x \in V_{k}^{\text {ss }}$ such that $\operatorname{GL}(n)_{k_{v}} x=$ $\mathrm{GL}(n)_{k_{v}}\left(\left(\operatorname{det} M_{x_{v}}\right) x_{v}\right)$ for all $v \in \mathfrak{M}$. So $G_{k_{v}} x=G_{k_{v}} x_{v}$ for all $v \in \mathfrak{M}$. This implies that $\Phi$ is a surjective map to the set of elements $\left(x_{v}\right)$ which satisfy the two conditions in Proposition 6.6.

Proposition 6.6 is a simple application of Theorem 6.2, which is classical and famous. We now explain the significance of Proposition 6.6 in comparison with Theorem 6.2. Theorem 6.2 contains a global condition on $d_{v}$, whereas Proposition 6.6 contains only a local condition for $\widetilde{S}_{v}$ except for the condition (2). So $G_{k} \backslash V_{k}^{\text {ss }}$ is nearly equal to $\prod_{v} G_{k_{v}} \backslash V_{k_{v}}^{\text {ss }}$ by Proposition 6.6. 
In this series of papers, we use Proposition 6.6 for the following purpose. In Part II, we define a Dirichlet series $\widetilde{Z}(s)$, which plays an important role in the proof of the main theorem, as a certain sum over $G_{k} \backslash V_{k}^{\text {ss }}$. We make $\widetilde{Z}(s)$ into a sum of two Euler products. In this process, we use Proposition 6.6 in order to modify the sum over $G_{k} \backslash V_{k}^{\text {ss }}$ to a sum over $\prod_{v} G_{k_{v}} \backslash V_{k_{v}}^{\text {ss }}$. Even though Proposition 6.6 contains the global condition (2) on $\widetilde{S}_{v}$, we use a technique in [11] and remove the condition (2) in the process of making $\widetilde{Z}(s)$ into a sum of two Euler products. In this manner, the use of the group $\mathrm{GL}(1) \times \mathrm{GL}(n)$ in Proposition 6.6 is more convenient than the use of the group $\operatorname{GL}(n)$ in Theorem 6.2 for our purposes.

\section{$\S 7 . \quad$ Measures on Orthogonal Groups of Orbital Representatives at Finite Places}

Let $v \in \mathfrak{M}_{\mathrm{f}}$ and $w_{v, \text { i }}^{\prime}$ be an alternative orbital representative of $G_{k_{v}} \backslash V_{k_{v}}^{\text {ss }}$ defined in (4.18). The main purpose of this section is to define measures on $\operatorname{GO}\left(w_{v, \text { i }}^{\prime}\right)_{k_{v}}^{\circ}, \operatorname{SO}\left(w_{v, \text { i }}^{\prime}\right)_{k_{v}}$ and $\operatorname{PGO}\left(w_{v, \text { i }}^{\prime}\right)_{k_{v}}^{\circ}$ essentially (but not directly) using their Iwasawa decompositions. The Iwasawa decompositions of these groups are well-known (see [24]). We review them briefly for the sake of the reader later in this section.

In this section also, we use the notations $w_{v, \dot{\mathrm{i}}}^{0}, w_{v, \mathrm{i}}^{\prime}$ (Definitions $4.9,4.14$ and (4.18)) and $T_{n}, N_{n}$ (at the beginning of $\S 5$ ).

Let $\widetilde{T}$ be as in (3.4). We identify $\widetilde{T}$ with the center of $\operatorname{GO}(x)$ for all $x \in V^{\mathrm{ss}}$ by Lemma 3.9. We define subgroups $T_{n}\left(w_{v, \dot{\mathrm{i}}}^{\prime}\right), \bar{T}_{n}\left(w_{v, \dot{\mathrm{i}}}^{\prime}\right)$ and $\widetilde{T}_{n}\left(w_{v, \dot{\mathrm{i}}}^{\prime}\right)$ respectively of $\operatorname{GO}\left(w_{v, \text { in }}^{\prime}\right)^{\circ}, \operatorname{SO}\left(w_{v, \dot{\mathrm{i}}}^{\prime}\right)$ and $\operatorname{PGO}\left(w_{v, \mathrm{i}}^{\prime}\right)^{\circ}$ by

$$
\begin{aligned}
& T_{n}\left(w_{v, \mathrm{i}}^{\prime}\right)=\left(\operatorname{GO}\left(w_{v, \mathrm{i}}^{\prime}\right) \cap T_{n}\right)^{\circ}, \bar{T}_{n}\left(w_{v, \mathrm{i}}^{\prime}\right)=\left(\operatorname{SO}\left(w_{v, \mathrm{i}}^{\prime}\right) \cap T_{n}\right)^{\circ}, \\
& \widetilde{T}_{n}\left(w_{v, \text { i }}^{\prime}\right)=T_{n}\left(w_{v, \text { i }}^{\prime}\right) / \widetilde{T} .
\end{aligned}
$$

Let $Z_{n}\left(w_{v, \mathrm{i}}^{\prime}\right), \bar{Z}_{n}\left(w_{v, \dot{\mathrm{i}}}^{\prime}\right)$ and $\widetilde{Z}_{n}\left(w_{v, \mathrm{i}}^{\prime}\right)$ be the centralizers of $T_{n}\left(w_{v, \mathrm{i}}^{\prime}\right), \bar{T}_{n}\left(w_{v, \dot{\mathrm{i}}}^{\prime}\right)$ and $\widetilde{T}_{n}\left(w_{v, \dot{\mathrm{i}}}^{\prime}\right)$ in $\operatorname{GO}\left(w_{v, \mathrm{i}}^{\prime}\right)^{\circ}, \mathrm{SO}\left(w_{v, \mathrm{i}}^{\prime}\right)$ and $\operatorname{PGO}\left(w_{v, \mathrm{i}}^{\prime}\right)^{\circ}$ respectively.

The groups $\operatorname{GO}\left(w_{v, \mathrm{i}}^{\prime}\right)^{\circ}$ and $\mathrm{SO}\left(w_{v, \mathrm{i}}^{\prime}\right)$ contain the following matrices:

$$
\begin{aligned}
& n_{1}\left(u_{1}\right)=\left(\begin{array}{ccc}
I_{m} & 0 & 0 \\
w_{v, \mathrm{i}}^{0}{ }^{t} u_{1} & I_{m_{0}} & 0 \\
v_{1} & u_{1} & I_{m}
\end{array}\right)\left(\begin{array}{l}
u_{1} \in \mathrm{M}\left(m, m_{0}\right), \\
v_{1}=\left(v_{1 i j}\right) \in \mathrm{M}(m, m), \\
v_{1 i j}=0 \text { for } i<j, v_{1}+{ }^{t} v_{1}=2 w_{v, \dot{\mathrm{I}}}^{0}\left[u_{1}\right]
\end{array}\right), \\
& n_{2}\left(u_{2}\right)=\left(\begin{array}{ccc}
I_{m} & 0 & 0 \\
0 & I_{m_{0}} & 0 \\
u_{2} & 0 & I_{m}
\end{array}\right) \quad\left(u_{2} \in \mathrm{M}(m, m), u_{2}+{ }^{t} u_{2}=0\right) \text {, }
\end{aligned}
$$




$$
n_{3}\left(u_{3}\right)=\left(\begin{array}{ccc}
u_{3} & 0 & 0 \\
0 & I_{m_{0}} & 0 \\
0 & 0 & { }^{t} u_{3}^{-1}
\end{array}\right) \quad\left(u_{3} \in N_{m}\right) .
$$

Let $N_{n}\left(w_{v, \text { in }}^{\prime}\right)$ be the subgroup of $\mathrm{GO}\left(w_{v, i}^{\prime}\right)^{\circ}$ generated by these elements. We regard $N_{n}\left(w_{v, \mathrm{i}}^{\prime}\right)$ as a subgroup of $\mathrm{SO}\left(w_{v, i}^{\prime}\right)$ and $\operatorname{PGO}\left(w_{v, i}^{\prime}\right)^{\circ}$. It is easy to see that $T_{n}\left(w_{v, \mathrm{i}}^{\prime}\right)$ and $Z_{n}\left(w_{v, \mathrm{i}}^{\prime}\right) N_{n}\left(w_{v, \mathrm{i}}^{\prime}\right)$, etc., are a maximal split torus and a minimal parabolic subgroup of $\mathrm{GO}\left(w_{v, \mathrm{i}}^{\prime}\right)^{\circ}$, etc.

Let $K_{n, v}$ be as in (5.1). We define subgroups $K_{n}\left(w_{v, \mathrm{i}}^{\prime}\right)$ and $\bar{K}_{n}\left(w_{v, \mathrm{i}}^{\prime}\right)$ respectively of $\mathrm{GO}\left(w_{v, \mathrm{i}}^{\prime}\right)_{k_{v}}^{\circ}$ and of $\mathrm{SO}\left(w_{v, \mathrm{i}}^{\prime}\right)_{k_{v}}$ as follows:

$$
K_{n}\left(w_{v, \mathrm{i}}^{\prime}\right)=\operatorname{GO}\left(w_{v, \mathrm{i}}^{\prime}\right)_{k_{v}}^{\circ} \cap K_{n, v}, \quad \bar{K}_{n}\left(w_{v, \mathrm{i}}^{\prime}\right)=\operatorname{SO}\left(w_{v, \mathrm{i}}^{\prime}\right)_{k_{v}} \cap K_{n, v} .
$$

Let $\widetilde{K}_{n}\left(w_{v, \mathrm{i}}^{\prime}\right)$ be the image of $K_{n}\left(w_{v, \mathrm{i}}^{\prime}\right)$ in $\operatorname{PGO}\left(w_{v, \mathrm{i}}^{\prime}\right)_{k_{v}}^{\circ}$.

Clearly $K_{n}\left(w_{v, \mathrm{i}}^{\prime}\right)$, etc., are open compact subgroups of $\mathrm{GO}\left(w_{v, \mathrm{i}}^{\prime}\right)_{k_{v}}^{\circ}$, etc.

Proposition 7.1. Let $v \in \mathfrak{M}_{\mathrm{f}}$ and $w_{v, \mathrm{i}}^{\prime}$ be an alternative orbital representative of $G_{k_{v}} \backslash V_{k_{v}}^{\text {ss }}$. Then we have the following decompositions:

$\mathrm{GO}\left(w_{v, \mathrm{i}}^{\prime}\right)_{k_{v}}^{\circ}=K_{n}\left(w_{v, \mathrm{i}}^{\prime}\right) Z_{n}\left(w_{v, \mathrm{i}}^{\prime}\right)_{k_{v}} K_{n}\left(w_{v, \mathrm{i}}^{\prime}\right)=K_{n}\left(w_{v, \mathrm{i}}^{\prime}\right) Z_{n}\left(w_{v, \mathrm{i}}^{\prime}\right)_{k_{v}} N_{n}\left(w_{v, \mathrm{i}}^{\prime}\right)_{k_{v}}$, $\mathrm{SO}\left(w_{v, \mathrm{i}}^{\prime}\right)_{k_{v}}=\bar{K}_{n}\left(w_{v, \mathrm{i}}^{\prime}\right) \bar{Z}_{n}\left(w_{v, \mathrm{i}}^{\prime}\right)_{k_{v}} \bar{K}_{n}\left(w_{v, \mathrm{i}}^{\prime}\right)=\bar{K}_{n}\left(w_{v, \mathrm{i}}^{\prime}\right) \bar{Z}_{n}\left(w_{v, \mathrm{i}}^{\prime}\right)_{k_{v}} N_{n}\left(w_{v, \mathrm{i}}^{\prime}\right)_{k_{v}}$, $\operatorname{PGO}\left(w_{v, \mathrm{i}}^{\prime}\right)_{k_{v}}^{\circ}=\widetilde{K}_{n}\left(w_{v, \mathrm{i}}^{\prime}\right) \widetilde{Z}_{n}\left(w_{v, \mathrm{i}}^{\prime}\right)_{k_{v}} \widetilde{K}_{n}\left(w_{v, \mathrm{i}}^{\prime}\right)=\widetilde{K}_{n}\left(w_{v, \mathrm{i}}^{\prime}\right) \widetilde{Z}_{n}\left(w_{v, \mathrm{i}}^{\prime}\right)_{k_{v}} N_{n}\left(w_{v, \mathrm{i}}^{\prime}\right)_{k_{v}}$.

We call the above decompositions the Cartan decomposition and the Iwasawa decomposition for each case.

Now we briefly review how to prove Proposition 7.1 using Conditions 5.9 and 5.10. We only consider the Cartan decompositions of $\operatorname{GO}\left(w_{v, \mathrm{i}}^{\prime}\right)_{k_{v}}^{\circ}$ for $w_{v, \mathrm{i}}^{\prime}$ such that $m_{0} \neq 0$ since the proof is similar and easier for the case $m_{0}=0$.

We express $X \in \mathrm{GO}\left(w_{v, \mathrm{i}}^{\prime}\right)_{k_{v}}^{\circ}$ using blocks $\left\{X_{i j}\right\}_{1 \leq i, j \leq 5}$ as follows:

$$
\left.X=\begin{array}{lllll}
1 & m-1 & m_{0} & 1 & m-1 \\
& m-1 \\
& 1 \\
& m-1 \\
X_{11} & X_{12} & X_{13} & X_{14} & X_{15} \\
X_{21} & X_{22} & X_{23} & X_{24} & X_{25} \\
X_{31} & X_{32} & X_{33} & X_{34} & X_{35} \\
X_{41} & X_{42} & X_{43} & X_{44} & X_{45} \\
X_{51} & X_{52} & X_{53} & X_{54} & X_{55}
\end{array}\right) .
$$

Multiplying elements of $K_{n}\left(w_{v, \mathrm{i}}^{\prime}\right)$ from both sides, if necessary, we may assume that

$$
X_{44}^{-1}\left(\begin{array}{llll}
X_{11} & X_{12} & X_{14} & X_{15} \\
X_{21} & X_{22} & X_{24} & X_{25} \\
X_{41} & X_{42} & X_{44} & X_{45} \\
X_{51} & X_{52} & X_{54} & X_{55}
\end{array}\right) \in \mathrm{M}(2 m, 2 m)_{\mathcal{O}_{v}}
$$


Then we obtain $w_{v \text {, } \mathrm{i}}^{0}\left[X_{43}\right]-2 X_{41}{ }^{t} X_{44}-2 X_{42}{ }^{t} X_{45}=0$ using the condition $X \in$ $\operatorname{GO}\left(w_{v, \mathrm{i}}^{\prime}\right)_{k_{v}}^{\circ}$. This implies $w_{v, \mathrm{i}}^{0}\left[X_{44}^{-1} X_{43}\right]=2 X_{44}^{-1} X_{41}+2 X_{44}^{-1} X_{42}{ }^{t}\left(X_{44}^{-1} X_{45}\right) \in$ $2 \mathcal{O}_{v}$. So, by Condition 5.10, $X_{44}^{-1} X_{43} \in \mathcal{O}_{v}^{m_{0}}$. Here, we used Condition 5.10 in an essential manner. For general $x \in V_{k_{v}}^{\mathrm{ss}}, \mathrm{GO}(x)_{k_{v}}^{\circ}$ (resp. $\left.\mathrm{SO}(x)_{k_{v}}\right)$ may not have the Iwasawa decomposition and the Cartan decomposition with $\mathrm{GO}(x)_{k_{v}}^{\circ} \cap$ $K_{n, v}$ (resp. $\mathrm{SO}(x)_{k_{v}} \cap K_{n, v}$ ) as the maximal compact subgroup. The essential reason why $\mathrm{GO}\left(w_{v, \mathrm{i}}^{\prime}\right)_{k_{v}}^{\circ}$ and $\mathrm{SO}\left(w_{v, \mathrm{i}}^{\prime}\right)_{k_{v}}$ have the decompositions in Proposition 7.1 is that $w_{v, \mathrm{i}}^{\prime}$ satisfies Condition 5.10.

Since $X \in \mathrm{GO}\left(w_{v, \mathrm{i}}^{\prime}\right)$, it follows easily that $X \in \mathrm{GO}\left(\left(w_{v, \mathrm{i}}^{\prime}\right)^{-1}\right)$. Considering its $(4,4)$-block, one can deduce that ${ }^{t}\left(X_{44}^{-1} X_{34}\right)\left(w_{v, \mathrm{i}}^{0}\right)^{-1} \in \mathcal{O}_{v}^{m_{0}}$. This implies that ${ }^{t}\left(X_{44}^{-1} X_{34}\right) \in \mathcal{O}_{v}^{m_{0}}$. Using these conditions, one can multiply suitable elements of $K_{n}\left(w_{v, \mathrm{i}}^{\prime}\right)$ from both sides and make $X$ in the following form:

$$
X=\left(\begin{array}{ccccc}
X_{11} & X_{12} & X_{13} & X_{14} & X_{15} \\
0 & X_{22} & X_{23} & 0 & X_{25} \\
0 & X_{32} & X_{33} & 0 & X_{35} \\
0 & 0 & 0 & X_{44} & 0 \\
0 & X_{52} & X_{53} & 0 & X_{55}
\end{array}\right) .
$$

After eliminating $X_{23}, X_{25}, X_{32}, X_{35}, X_{52}, X_{53}$ by induction on $m$, one can eliminate $X_{12}, \cdots, X_{15}$ also. Thus, we obtain the Cartan decomposition.

Definition 7.4. Let $v \in \mathfrak{M}_{\mathrm{f}}$. We define invariant measures $d g_{v, \mathrm{i}}^{\prime \prime}, d \bar{g}_{v, \mathrm{i}}^{\prime \prime}$ and $d \widetilde{g}_{v, \mathrm{i}}^{\prime \prime}$ respectively on $\operatorname{GO}\left(w_{v, \mathrm{i}}^{\prime}\right)_{k_{v}}^{\circ}, \operatorname{SO}\left(w_{v, \mathrm{i}}^{\prime}\right)_{k_{v}}$ and $\operatorname{PGO}\left(w_{v, \mathrm{i}}^{\prime}\right)_{k_{v}}^{\circ}$ so that

$$
\int_{K_{n}\left(w_{v, \mathrm{i}}^{\prime}\right)} d g_{v, \mathrm{i}}^{\prime \prime}=1, \quad \int_{\bar{K}_{n}\left(w_{v, \mathrm{i}}^{\prime}\right)} d \bar{g}_{v, \mathrm{i}}^{\prime \prime}=1, \quad \int_{\widetilde{K}_{n}\left(w_{v, \mathrm{i}}^{\prime}\right)} d \widetilde{g}_{v, \mathrm{i}}^{\prime \prime}=1 .
$$

Since $\operatorname{GO}\left(w_{v, \mathrm{i}}^{\prime}\right)_{k_{v}}^{\circ}$ has the Iwasawa decomposition with $K_{n}\left(w_{v, \mathrm{i}}^{\prime}\right)$ as the maximal compact subgroup, $K_{n}\left(w_{v, \mathrm{i}}^{\prime}\right)$ is called the special maximal compact subgroup. Even though we did not use the Iwasawa decomposition to define a measure on $\operatorname{GO}\left(w_{v, \mathrm{i}}^{\prime}\right)_{k_{v}}^{\circ}$ for $v \in \mathfrak{M}_{\mathrm{f}}$ directly, Proposition 7.1 tells us that our choice of the measure is natural in some sense. The situation is similar for $\mathrm{SO}\left(w_{v, \mathrm{i}}^{\prime}\right)_{k_{v}}$ and $\operatorname{PGO}\left(w_{v, \mathrm{i}}^{\prime}\right)_{k_{v}}^{\circ}$.

\section{$\S 8$. Canonical Measures on Orthogonal Groups}

The main purpose of this section is to define invariant measures on orthogonal groups locally and globally which are canonical in some sense. We have defined measures on $\mathrm{GO}(x)_{k_{v}}^{\circ}, \mathrm{SO}(x)_{k_{v}}, \mathrm{PGO}(x)_{k_{v}}^{\circ}$ for alternative orbital 
representatives $x$ and $v \in \mathfrak{M}_{\mathrm{f}}$. We shall define measures on $\mathrm{GO}(x)_{k_{v}}^{\circ}, \mathrm{SO}(x)_{k_{v}}$, and $\operatorname{PGO}(x)_{k_{v}}^{\circ}$ for standard orbital representatives $x$ and $v \in \mathfrak{M}_{\infty}$ in Part II. In the following, we consider $v \in \mathfrak{M}_{\mathrm{f}}$, but the argument is similar for $v \in \mathfrak{M}_{\infty}$ where we use $w_{v, \text { in }}$ instead of $w_{v, \text { in }}^{\prime}$.

We first consider the local situation. Suppose that $x=\alpha_{x} w_{v, \mathrm{i}}^{\prime} \in V_{k_{v}}^{\mathrm{ss}}$ for $\alpha_{x}=\left(t_{0}, g_{1}\right) \in G_{k_{v}}$. Let

$$
\varphi_{\alpha_{x}}: G_{k_{v}} \ni h_{x} \mapsto \alpha_{x}^{-1} h_{x} \alpha_{x}=g_{1}^{-1} h_{x} g_{1} \in G_{k_{v}} .
$$

It is easy to see that $\varphi_{\alpha_{x}}\left(G_{x k_{v}}\right)=G_{w_{v, \mathrm{i}}^{\prime} k_{v}}$. So $\varphi_{\alpha_{x}}$ induces a map $G_{k_{v}} / G_{x k_{v}}^{\circ} \rightarrow$ $G_{k_{v}} / G_{w_{v, \mathrm{i}}^{\prime} k_{v}}^{\circ}$. Also $\varphi_{\alpha_{x}}$ induces a homomorphism $\widetilde{G}_{x k_{v}}^{\circ} \rightarrow \widetilde{G}_{w_{v, \mathrm{i}}^{\prime} k_{v}}^{\circ}$. We denote these maps also by $\varphi_{\alpha_{x}}$.

Now we use the identification $G_{x} \cong \mathrm{GO}(x)$, etc., (see Lemma 3.9). We define a measure $d g_{x, v}^{\prime \prime}$ on $G_{x k_{v}}^{\circ} \cong \mathrm{GO}(x)_{k_{v}}^{\circ}$ as follows:

$$
d g_{x, v}^{\prime \prime}=\left(\varphi_{\alpha_{x}}\right)^{*}\left(d g_{v, \mathrm{i}}^{\prime \prime}\right),
$$

i.e., $d g_{x, v}^{\prime \prime}$ is the pullback of $d g_{v, \mathrm{i}}^{\prime \prime}$ by $\varphi_{\alpha_{x}}$. Since $d g_{v, \mathrm{i}}^{\prime \prime}$ is an invariant measure on $\operatorname{GO}\left(w_{v, \mathrm{i}}^{\prime}\right)_{k_{v}}^{\circ}, d g_{x, v}^{\prime \prime}$ is an invariant measure on $\operatorname{GO}(x)_{k_{v}}^{\circ}$. We define invariant measures $d \bar{g}_{x, v}^{\prime \prime}, d \widetilde{g}_{x, v}^{\prime \prime}$ on $\mathrm{SO}(x)_{k_{v}}, \mathrm{PGO}(x)_{k_{v}}^{\circ}$ similarly.

Lemma 8.2. The measures $d g_{x, v}^{\prime \prime}, d \bar{g}_{x, v}^{\prime \prime}$ and $d \widetilde{g}_{x, v}^{\prime \prime}$ do not depend on the choice of $\alpha_{x}$.

Proof. Suppose that $\alpha_{x}=\left(t_{0}, g_{1}\right), \alpha_{x}^{\prime}=\left(t_{0}^{\prime}, g_{1}^{\prime}\right) \in G_{k_{v}}$ and $x=\alpha_{x} w_{v, \mathrm{i}}^{\prime}$ $=\alpha_{x}^{\prime} w_{v, \mathrm{i}}^{\prime}$. It is easy to see that $\alpha_{x}^{-1} \alpha_{x}^{\prime} \in G_{w_{v, \mathrm{i}}^{\prime} k_{v}}$ and $g_{1}^{-1} g_{1}^{\prime} \in \operatorname{GO}\left(w_{v, \mathrm{i}}^{\prime}\right)_{k_{v}}$.

Let $h=\alpha_{x}^{-1} \alpha_{x}^{\prime}$ and $h_{1}=g_{1}^{-1} g_{1}^{\prime}$. Let $\varphi_{\alpha_{x}}$ and $\varphi_{\alpha_{x}^{\prime}}$ be the maps which were defined in (8.1). Then, for $h_{x} \in \mathrm{GO}(x)_{k_{v}}^{\circ}$,

$$
\varphi_{\alpha_{x}^{\prime}}\left(h_{x}\right)=\alpha_{x}^{\prime-1} h_{x} \alpha_{x}^{\prime}=\left(\alpha_{x} h\right)^{-1} h_{x}\left(\alpha_{x} h\right)=h^{-1} \alpha_{x}^{-1} h_{x} \alpha_{x} h=\varphi_{h} \circ \varphi_{\alpha_{x}}\left(h_{x}\right)
$$

where

$$
\varphi_{h}: \mathrm{GO}\left(w_{v, \mathrm{i}}^{\prime}\right)_{k_{v}}^{\circ} \ni h_{w_{v, \mathrm{i}}^{\prime}} \mapsto h^{-1} h_{w_{v, \mathrm{i}}^{\prime}} h=h_{1}^{-1} h_{w_{v, \mathrm{i}}^{\prime}} h_{1} \in \mathrm{GO}\left(w_{v, \mathrm{i}}^{\prime}\right)_{k_{v}}^{\circ} .
$$

So

$$
\left(\varphi_{\alpha_{x}^{\prime}}\right)^{*}\left(d g_{v, \mathrm{i}}^{\prime \prime}\right)=\left(\varphi_{h} \circ \varphi_{\alpha_{x}}\right)^{*}\left(d g_{v, \mathrm{i}}^{\prime \prime}\right)=\left(\varphi_{\alpha_{x}}\right)^{*} \circ\left(\varphi_{h}\right)^{*}\left(d g_{v, \mathrm{i}}^{\prime \prime}\right) .
$$

By Lemma 3.11 and the comment after that, $\left[\mathrm{GO}\left(w_{v, \mathrm{i}}^{\prime}\right)_{k_{v}}: \operatorname{GO}\left(w_{v, \mathrm{i}}^{\prime}\right)_{k_{v}}^{\circ}\right]$ is 1 or 2 according as $n$ is odd or even. If $n$ is even then there exists $\tau_{v, \mathrm{i}} \in$ $\mathrm{GO}\left(w_{v, \mathrm{i}}^{\prime}\right)_{k_{v}} \backslash \mathrm{GO}\left(w_{v, \mathrm{i}}^{\prime}\right)_{k_{v}}^{\circ}$ such that $\tau_{v, \mathrm{i}}^{2}=I_{n}$ by Proposition 4.22. Since the order of $\tau_{v, \mathrm{i}}$ is finite, the map

$$
\varphi_{\tau_{v, \mathrm{i}}}: \operatorname{GO}\left(w_{v, \mathrm{i}}^{\prime}\right)_{k_{v}}^{\circ} \ni h_{w_{v, \mathrm{i}}^{\prime}} \mapsto \tau_{v, \mathrm{i}}^{-1} h_{w_{v, \mathrm{i}}^{\prime}} \tau_{v, \mathrm{i}} \in \operatorname{GO}\left(w_{v, \mathrm{i}}^{\prime}\right)_{k_{v}}^{\circ}
$$


is measure preserving. Since $d g_{v, \mathrm{i}}^{\prime \prime}$ is a unimodular measure on $\operatorname{GO}\left(w_{v, \mathrm{i}}^{\prime}\right)_{k_{v}}^{\circ}$, the map

$$
\varphi_{h_{1}^{0}}: \operatorname{GO}\left(w_{v, \mathrm{i}}^{\prime}\right)_{k_{v}}^{\circ} \ni h_{w_{v, \mathrm{i}}^{\prime}} \mapsto\left(h_{1}^{0}\right)^{-1} h_{w_{v, \mathrm{i}}^{\prime}} h_{1}^{0} \in \mathrm{GO}\left(w_{v, \mathrm{i}}^{\prime}\right)_{k_{v}}^{\circ}
$$

for $h_{1}^{0} \in \mathrm{GO}\left(w_{v, \mathrm{i}}^{\prime}\right)_{k_{v}}^{\circ}$ is also measure preserving. Therefore, $\left(\varphi_{h}\right)^{*}\left(d g_{v, \mathrm{i}}^{\prime \prime}\right)=d g_{v, \mathrm{i}}^{\prime \prime}$ for $h \in \operatorname{GO}\left(w_{v, \mathrm{i}}^{\prime}\right)_{k_{v}}$ for all $n \geq 2$. Thus, $\left(\varphi_{\alpha_{x}^{\prime}}\right)^{*}\left(d g_{v, \mathrm{i}}^{\prime \prime}\right)=\left(\varphi_{\alpha_{x}}\right)^{*}\left(d g_{v, \mathrm{i}}^{\prime \prime}\right)$.

The proof is similar for $d \bar{g}_{x, v}^{\prime \prime}, d \widetilde{g}_{x, v}^{\prime \prime}$.

We continue to identify $\widetilde{T}$ with the center $\left\{\tilde{t}_{0} I_{n} \mid \tilde{t}_{0} \in \mathrm{GL}(1)\right\}$ of $\operatorname{GO}(x)^{\circ}$. Let $d^{\times} \tilde{t}_{0}$ be the usual measure on $k_{v}^{\times} \cong \widetilde{T}_{k_{v}}$.

Proposition 8.3. Suppose that $v \in \mathfrak{M}_{\mathrm{f}}$.

(1) If $x \in V_{k_{v}}^{\mathrm{ss}}$ then $d g_{x, v}^{\prime \prime}=d \widetilde{g}_{x, v}^{\prime \prime} d^{\times} \tilde{t}_{0}$.

(2) If $n \geq 3$ is odd and $x \in V_{k_{v}}^{\mathrm{ss}}$ then $d \widetilde{g}_{x, v}^{\prime \prime}=d \bar{g}_{x, v}^{\prime \prime}$.

Proof. It is enough to consider standard representatives. Let $x \in V_{k_{v}}^{\text {ss }}$ be a standard representative. We first discuss the statement (1).

Let $f$ be the characteristic function of $K_{v} \cap G_{x k_{v}}^{\circ} \subseteq G_{x k_{v}}^{\circ}$. We compute the integral of $f$ with respect to the two measures $d g_{x, v}^{\prime \prime}$ and $d \widetilde{g}_{x, v}^{\prime \prime} d^{\times} \tilde{t}_{0}$. It follows from the definition that $\int_{G_{x, v}^{\circ}} f\left(g_{x, v}^{\prime \prime}\right) d g_{x, v}^{\prime \prime}=1$. Let $g_{x, v}^{\prime \prime} \in G_{x k_{v}}^{\circ}$ and $\left(\widetilde{t}_{0}^{-2}, \widetilde{t}_{0} I_{n}\right) \in \widetilde{T}_{k_{v}}$. It is easy to see that $f\left(g_{x, v}^{\prime \prime}\left(\widetilde{t}_{0}^{-2}, \widetilde{t}_{0} I_{n}\right)\right)=0$ unless $g_{x, v}^{\prime \prime} \in\left(K_{v} \cap\right.$ $\left.G_{x k_{v}}^{\circ}\right) \widetilde{T}_{k_{v}}$. So we express the above $g_{x, v}^{\prime \prime}$ as $\kappa\left(q^{-2}, q I_{n}\right)$ for some $\kappa \in K_{v} \cap G_{x k_{v}}^{\circ}$ and $q \in k_{v}^{\times}$.

Since $d^{\times} \tilde{t}_{0}$ is an invariant measure on $\widetilde{T}_{k_{v}}$,

$$
\int_{\widetilde{T}_{k_{v}}} f\left(\kappa\left(q^{-2}, q I_{n}\right)\left(\widetilde{t}_{0}^{-2}, \widetilde{t}_{0} I_{n}\right)\right) d^{\times} \tilde{t}_{0}=\int_{\widetilde{T}_{k_{v}}} f\left(\kappa\left(\widetilde{t}_{0}^{-2}, \widetilde{t}_{0} I_{n}\right)\right) d^{\times} \tilde{t}_{0}
$$

Note that $f\left(\kappa\left(\widetilde{t}_{0}^{-2}, \widetilde{t}_{0} I_{n}\right)\right) \neq 0$ if and only if $t_{0} \in \mathcal{O}_{v}^{\times}$. So the value of the integral (8.4) is 1 . Hence

$$
\int_{G_{x k_{v}}^{\circ}} f\left(g_{x, v}^{\prime \prime}\left(\widetilde{t}_{0}^{-2}, \widetilde{t}_{0} I_{n}\right)\right) d \widetilde{g}_{x, v}^{\prime \prime} d^{\times} \tilde{t}_{0}=\int_{\left(K_{v} \cap G_{x k_{v}}^{\circ}\right) \widetilde{T}_{k_{v}} / \widetilde{T}_{k_{v}}} d \widetilde{g}_{x, v}^{\prime \prime} .
$$

Since the image of $K_{v} \cap G_{x k_{v}}^{\circ}$ in $\widetilde{G}_{x k_{v}}$ is $\left(K_{v} \cap G_{x k_{v}}^{\circ}\right) \widetilde{T}_{k_{v}} / \widetilde{T}_{k_{v}}$, the value of the above integral is 1 by the definition of $d \widetilde{g}_{x, v}^{\prime \prime}$. Since $d g_{x, v}^{\prime \prime}$ and $d \widetilde{g}_{x, v}^{\prime \prime} d^{\times} \tilde{t}_{0}$ are invariant measures on $G_{x k_{v}}^{\circ}, d g_{x, v}^{\prime \prime}=d \widetilde{g}_{x, v}^{\prime \prime} d^{\times} \tilde{t}_{0}$.

We next discuss the statement (2). It suffices to prove that, if $\left(\widetilde{t}_{0}^{-2}, \widetilde{t}_{0} I_{n}\right) \in$ $\widetilde{T}_{k_{v}}$ and $g \in \mathrm{SO}(x)_{k_{v}}$ satisfies $\left(\widetilde{t}_{0}^{-2}, \widetilde{t}_{0} I_{n}\right) g \in K_{v}$, then $\left(\widetilde{t}_{0}^{-2}, \widetilde{t}_{0} I_{n}\right), g \in K_{v}$. 
Note that, if $\left(\widetilde{t}_{0}^{-2}, \widetilde{t}_{0} I_{n}\right) g \in K_{v}, \widetilde{t}_{0}^{-2} \in \mathcal{O}_{v}^{\times}$. So $\widetilde{t}_{0} \in \mathcal{O}_{v}^{\times}$. Therefore, $g \in$ $K_{v} \cap \mathrm{SO}(x)_{k_{v}}$.

We shall prove a similar proposition in Part II for $v \in \mathfrak{M}_{\infty}$.

We call $d g_{x, v}^{\prime \prime}, d \bar{g}_{x, v}^{\prime \prime}$ and $d \widetilde{g}_{x, v}^{\prime \prime}$ the canonical measures respectively on $\mathrm{GO}(x)_{k_{v}}^{\circ}, \mathrm{SO}(x)_{k_{v}}$ and $\mathrm{PGO}(x)_{k_{v}}^{\circ}$.

Definition 8.5. For $x \in V_{k}^{\mathrm{ss}}$, we define measures $d g_{x, \mathbb{A}}^{\prime \prime}, d \bar{g}_{x, \mathbb{A}}^{\prime \prime}$ and $d \widetilde{g}_{x, \mathbb{A}}^{\prime \prime}$ respectively on $\mathrm{GO}(x)_{\mathbb{A}}^{\circ}, \mathrm{SO}(x)_{\mathbb{A}}$ and $\mathrm{PGO}(x)_{\mathbb{A}}^{\circ}$ as follows:

$$
d g_{x, \mathbb{A}}^{\prime \prime}=\prod_{v \in \mathfrak{M}} d g_{x, v}^{\prime \prime}, \quad d \bar{g}_{x, \mathbb{A}}^{\prime \prime}=\prod_{v \in \mathfrak{M}} d \bar{g}_{x, v}^{\prime \prime}, \quad d \widetilde{g}_{x, \mathbb{A}}^{\prime \prime}=\prod_{v \in \mathfrak{M}} d \widetilde{g}_{x, v}^{\prime \prime} .
$$

We call these measures the canonical measures also.

Lemma 8.7. Let $x \in V_{k}^{\mathrm{ss}}$. Then, for all but finitely many $v \in \mathfrak{M}_{\mathrm{f}}$,

$$
\int_{\mathrm{GO}(x)_{k_{v}}^{\circ} \cap \mathrm{GL}(n)_{\mathcal{O}_{v}}} d g_{x, v}^{\prime \prime}=1, \quad \int_{\mathrm{SO}(x)_{k_{v}} \cap \mathrm{GL}(n)_{\mathcal{O}}} d \bar{g}_{x, v}^{\prime \prime}=1 .
$$

Proof. Since the argument is similar for $d \bar{g}_{x, v}^{\prime \prime}$, we only consider $d g_{x, v}^{\prime \prime}$. Suppose that $x=\alpha_{x, v} w_{v, \mathrm{i}}^{\prime}$ where $\alpha_{x, v} \in G_{k_{v}}$ and $w_{v, \mathrm{i}}^{\prime}$ is an alternative orbital representative. For all but finitely many $v \in \mathfrak{M}_{\mathrm{f}}, x \in V_{\mathcal{O}_{v}}$ and $\operatorname{ord}_{v}(P(x))=0$. Then $x$ is unramified (see Definition 4.19), which implies that $\operatorname{ord}_{v}\left(P\left(w_{v, \text { i }}^{\prime}\right)\right)=$ 0 . We only consider such $v \in \mathfrak{M}_{\mathrm{f}}$.

Since $w_{v, \mathrm{i}}^{\prime}$ satisfies Condition 5.8, $\alpha_{x, v} \in K_{v} G_{w_{v, \mathrm{i}}^{\prime} k_{v}}$. So we may assume that $\alpha_{x, v} \in K_{v}$. By the definition of $d g_{x, v}^{\prime \prime}$,

$$
\begin{aligned}
\int_{\mathrm{GO}(x)_{k_{v}}^{\circ} \cap \mathrm{GL}(n)_{\mathcal{O}_{v}}} d g_{x, v}^{\prime \prime} & =\int_{\varphi_{\alpha_{x, v}}\left(\mathrm{GO}(x)_{k_{v}}^{\circ} \cap \mathrm{GL}(n)_{\mathcal{O}_{v}}\right)} d g_{v, \mathrm{i}}^{\prime \prime} \\
& =\int_{\alpha_{x, v}^{-1}\left(\operatorname{GO}(x)_{k_{v}}^{\circ} \cap \mathrm{GL}(n)_{\mathcal{O}_{v}}\right) \alpha_{x, v}} d g_{v, \mathrm{i}}^{\prime \prime} .
\end{aligned}
$$

Since $\alpha_{x, v} \in K_{v}$

$$
\alpha_{x, v}^{-1}\left(\operatorname{GO}(x)_{k_{v}}^{\circ} \cap \mathrm{GL}(n)_{\mathcal{O}_{v}}\right) \alpha_{x, v}=\mathrm{GO}\left(w_{v, \mathrm{i}}^{\prime}\right)_{k_{v}}^{\circ} \cap \mathrm{GL}(n)_{\mathcal{O}_{v}}=K_{n}\left(w_{v, \mathrm{i}}^{\prime}\right) .
$$

So $(8.8)$ is equal to

$$
\int_{K_{n}\left(w_{v, \mathrm{i}}^{\prime}\right)} d g_{v, \mathrm{i}}^{\prime \prime}=1 .
$$

This completes the proof of the lemma.

Proposition 8.9. The measures $d g_{x, \mathbb{A}}^{\prime \prime}, d \bar{g}_{x, \mathbb{A}}^{\prime \prime}$ and $d \widetilde{g}_{x, \mathbb{A}}^{\prime \prime}$ are well-defined. 
Proof. Since

$$
G_{x \mathbb{A}_{\mathrm{f}}}^{\circ} \cap \prod_{v \in \mathfrak{M}_{\mathrm{f}}} K_{v} \cong \prod_{v \in \mathfrak{M}_{\mathrm{f}}} \mathrm{GO}(x)_{k_{v}}^{\circ} \cap \mathrm{GL}(n)_{\mathcal{O}_{v}}
$$

is an open compact subgroup of $G_{x \mathbb{A}_{\mathrm{f}}}^{\circ}$ and its volume with respect to the product measure $\prod_{v \in \mathfrak{M}_{\mathrm{f}}} d g_{x, v}^{\prime \prime}$ is a non-zero finite value, the measure $d g_{x, \mathbb{A}}^{\prime \prime}$ is well-defined by the above lemma. Similarly, the measure $d \bar{g}_{x, \mathbb{A}}^{\prime \prime}$ is well-defined.

Let $d_{\mathrm{pr}}^{\times} \widetilde{t}_{0}$ be the measure on $\widetilde{T}_{\mathbb{A}}$ which is the product of the usual measures on all $k_{v}^{\times}$. Then $d g_{x, \mathbb{A}}^{\prime \prime}=d \widetilde{g}_{x, \mathbb{A}}^{\prime \prime} d_{\mathrm{pr}}^{\times} \widetilde{t}_{0}$. Since $d_{\mathrm{pr}}^{\times} \widetilde{t}_{0}$ is well-defined, $d \widetilde{g}_{x, \mathbb{A}}^{\prime \prime}$ is welldefined also.

Definition 8.10. Let

$$
\operatorname{vol}\left(\widetilde{G}_{x \mathbb{A}}^{\circ} / \widetilde{G}_{x k}^{\circ}\right)=\int_{\widetilde{G}_{x \mathbb{A}}^{\circ} / \widetilde{G}_{x k}^{\circ}} d \widetilde{g}_{x, \mathbb{A}}^{\prime \prime}
$$

for $x \in V_{k}^{\mathrm{ss}}$. We call $\operatorname{vol}\left(\widetilde{G}_{x \mathbb{A}}^{\circ} / \widetilde{G}_{x k}^{\circ}\right)$ the unnormalized Tamagawa number of $\widetilde{G}_{x}^{\circ}$.

Note that if $n \geq 3$ is odd then $\widetilde{G}_{x}^{\circ}=\widetilde{G}_{x}$ can be identified with $\operatorname{SO}(x)$. In $\S 10$ and Part II, we shall compute the value of $\operatorname{vol}\left(\widetilde{G}_{x \mathbb{A}} / \widetilde{G}_{x k}\right)$ for odd $n$. The unnormalized Tamagawa number $\operatorname{vol}\left(\widetilde{G}_{x \mathbb{A}} / \widetilde{G}_{x k}\right)$ is not the Tamagawa number $\tau\left(\widetilde{G}_{x}\right)$ of $\operatorname{SO}(x)$ which we shall review in the next section. It turns out that $\operatorname{vol}\left(\widetilde{G}_{x \mathbb{A}} / \widetilde{G}_{x k}\right)$ is equal to $\tau\left(\widetilde{G}_{x}\right) \prod_{v \in \mathfrak{M}} \widetilde{c}_{v, x}^{\prime \prime}$ where $\widetilde{c}_{v, x}^{\prime \prime}$ is a local factor for $v \in \mathfrak{M}$ which can be expressed using the local densities of $\operatorname{SO}\left(w_{v, \dot{1}}^{\prime}\right)$ for the alternative orbital representatives $w_{v, \text { in }}^{\prime}$ such that $w_{v, \text { i }}^{\prime} \in G_{k_{v}} x$. The unnormalized Tamagawa number $\operatorname{vol}\left(\widetilde{G}_{x \mathbb{A}} / \widetilde{G}_{x k}\right)$ is an important invariant for $\widetilde{G}_{x} \cong \operatorname{SO}(x)$.

\section{§9. The Tamagawa Measure on $\widetilde{G}_{x \mathbb{A}}$}

In this section $n \geq 3$ is an odd integer. In this section we shall review some facts concerning the Tamagawa measure on $\widetilde{G}_{x \mathbb{A}}$ for $x \in V_{k}^{\text {ss }}$. Since $\widetilde{G}_{x} \cong \operatorname{SO}(x)$ for odd $n \geq 3$, these facts are well-known (see [28] and [32]).

We first define invariant measures both on $\widetilde{G}_{x k_{v}}$ and $\widetilde{G}_{x \mathbb{A}}$ using invariant differential forms on $G$ and $V^{\text {ss }}$ which are defined over the global field $k$. Let $g=\left(t_{0}, g_{1}\right) \in G_{k_{v}}$ and $y \in V_{k_{v}}^{\mathrm{ss}}$. We express elements $g_{1} \in \operatorname{GL}(n)_{k_{v}}$ and $y \in V_{k_{v}}^{\mathrm{ss}}$ as follows:

$$
g_{1}=\left(\begin{array}{ccc}
g_{11} & \cdots & g_{1 n} \\
\vdots & \ddots & \vdots \\
g_{n 1} & \cdots & g_{n n}
\end{array}\right), \quad y=\left(\begin{array}{cccc}
2 y_{11} & y_{12} & \cdots & y_{1 n} \\
y_{12} & 2 y_{22} & \ddots & \vdots \\
\vdots & \ddots & \ddots & y_{n-1 n} \\
y_{1 n} & \cdots & y_{n-1 n} & 2 y_{n n}
\end{array}\right)
$$


We first define an invariant measure on $G_{k_{v}}$. It is easy to see that

$$
\nu=t_{0}^{-1} d t_{0} \wedge\left(\operatorname{det} g_{1}\right)^{-n} \bigwedge_{1 \leq i, j \leq n} d g_{i j}
$$

is an invariant differential form on $G=\mathrm{GL}(1) \times \mathrm{GL}(n)$. Note that (9.1) is a differential form over the global field $k$. For $v \in \mathfrak{M}$, we define a measure $d \mu_{v}=d \mu_{v}(g)$ on $G_{k_{v}}$ as follows:

$$
d \mu_{v}=\left|t_{0}\right|_{v}^{-1} d t_{0}\left|\operatorname{det} g_{1}\right|_{v}^{-n} \prod_{1 \leq i, j \leq n} d g_{i j}
$$

where $d t_{0}$ and $d g_{i j}$ are the usual measures on $k_{v}$ which we have chosen in $\S 2$. Since (9.1) is an invariant differential form on $G_{k_{v}}, d \mu_{v}$ is an invariant measure on $G_{k_{v}}$. We call $d \mu_{v}$ the Tamagawa measure on $G_{k_{v}}$.

The following theorem is Theorem [28, p.118] with respect to the special case $\operatorname{GL}(n)$.

Theorem 9.3. Let $v \in \mathfrak{M}_{\mathrm{f}}$. Then, for any $\nu \in \mathbb{Z}_{>0}$,

$$
\int_{g_{1} \in \operatorname{GL}(n)_{\mathcal{O}_{v}}}\left|\operatorname{det} g_{1}\right|_{v}^{-n} \prod_{1 \leq i, j \leq n} d g_{i j}=\frac{\sharp \operatorname{GL}(n)_{\mathcal{O}_{v} / \mathfrak{p}_{v}^{\nu}}}{q_{v}^{\nu \operatorname{dim} \operatorname{GL}(n)}}=\prod_{j=1}^{n}\left(1-q_{v}^{-j}\right) \text {. }
$$

Note that $\frac{\sharp \mathrm{GL}(n)_{\mathcal{O}_{v} / \mathfrak{p}_{v}^{\nu}}}{q_{v}^{\nu \operatorname{dim} \mathrm{GL}(n)}}$ is the local density of $\mathrm{GL}(n)_{k_{v}}$ for $v \in \mathfrak{M}_{\mathrm{f}}$. Using the above lemma, we obtain

$$
\int_{K_{v}} d \mu_{v}=\left(1-q_{v}^{-1}\right) \prod_{j=1}^{n}\left(1-q_{v}^{-j}\right) .
$$

We next define invariant measures on $V_{k_{v}}^{\mathrm{ss}}$ and $G_{k_{v}} / G_{x k_{v}}$ for $x \in V_{k_{v}}^{\mathrm{ss}}$. For $v \in \mathfrak{M}$, we define a measure $d y$ on $V_{k_{v}}$ as follows:

$$
d y=\prod_{1 \leq i \leq j \leq n} d y_{i j}
$$

where $d y_{i j}$ is the usual measure on $k_{v}$. For the rest of this paper, we denote the volume of any measurable subset $U \subseteq V_{k_{v}}$ with respect to $d y$ by $\operatorname{vol}(U)$. By definition, $\operatorname{vol}\left(V_{\mathcal{O}_{v}}\right)=1$. It is easy to see that $\nu_{x}^{\prime}=P(y)^{-(n+1) / 2} \bigwedge_{1 \leq i \leq j \leq n} d y_{i j}$ is a $G$-invariant differential form over the global field $k$ on $V^{\text {ss }}$. So $|P(y)|_{v}^{-(n+1) / 2} d y$ is a $G_{k_{v}}$-invariant measure on $V_{k_{v}}$. For $x \in V_{k_{v}}^{\text {ss }}$, we define an invariant measure $d \mu_{x, v}^{\prime}=d \mu_{x, v}^{\prime}\left(g^{\prime}\right)$ on $G_{k_{v}} / G_{x k_{v}}$ so that

$$
\int_{g^{\prime} \in G_{k_{v}} / G_{x} k_{v}} F^{\prime}\left(g^{\prime} x\right) d \mu_{x, v}^{\prime}=\int_{y \in G_{k_{v}} x} F^{\prime}(y)|P(y)|_{v}^{-\frac{n+1}{2}} d y
$$


for all measurable functions $F^{\prime}$ on $V_{k_{v}}^{\text {ss }}$.

Now we define invariant measures on $G_{x k_{v}}$ and $\widetilde{G}_{x k_{v}}$ for $x \in V_{k_{v}}^{\text {ss }}$. On $\widetilde{T}_{k_{v}}=\left\{\left(\tilde{t}_{0}^{-2}, \tilde{t}_{0} I_{n}\right) \mid \tilde{t}_{0} \in k_{v}^{\times}\right\}, \tilde{t}_{0}^{-1} d \tilde{t}_{0}$ is an invariant differential form. So there exists an invariant differential form $\tilde{\nu}_{x}^{\prime \prime}$ on $\widetilde{G}_{x k_{v}}$ such that

$$
\nu=\left(\nu_{x}^{\prime} \wedge \tilde{\nu}_{x}^{\prime \prime}\right) \wedge\left(\tilde{t}_{0}^{-1} d \tilde{t}_{0}\right) .
$$

We may not be able to express $\tilde{\nu}_{x}^{\prime \prime}$ explicitly. However, using $\tilde{\nu}_{x}^{\prime \prime} \wedge\left(\tilde{t}_{0}^{-1} d \tilde{t}_{0}\right)$ and $\tilde{\nu}_{x}^{\prime \prime}$, we can define the following invariant measures $d \mu_{x, v}^{\prime \prime}$ and $d \tilde{\mu}_{x, v}^{\prime \prime}$ respectively on $G_{x k_{v}}$ and $\widetilde{G}_{x k_{v}}$. Let $d \mu_{\widetilde{T}, v}=d \mu_{\widetilde{T}, v}\left(\widetilde{t}_{0}\right)$ be the Tamagawa measure on $\widetilde{T}_{k_{v}} \cong \mathrm{GL}(1)_{k_{v}}$. Then

$$
\int_{\widetilde{T}_{k_{v}} \cap K_{v}} d \mu_{\widetilde{T}, v}=1-q_{v}^{-1}
$$

for $v \in \mathfrak{M}_{\mathrm{f}}$. Since $\prod_{v \in \mathfrak{M}_{\mathrm{f}}} \int_{\widetilde{T}_{k_{v}} \cap K_{v}} d \mu_{\widetilde{T}, v}$ does not converge absolutely, we consider the Tamagawa measure on $\widetilde{T}$ only locally.

Definition 9.7. Let $x \in V_{k_{v}}^{\text {ss }}$. We define an invariant measure $d \mu_{x, v}^{\prime \prime}=$ $d \mu_{x, v}^{\prime \prime}\left(g^{\prime \prime}\right)$ on $G_{x k_{v}}$ so that

$$
\int_{g \in G_{k_{v}}} F(g) d \mu_{v}=\int_{g^{\prime} \in G_{k_{v}} / G_{x k_{v}}}\left(\int_{g^{\prime \prime} \in G_{x k_{v}}} F\left(g^{\prime} g^{\prime \prime}\right) d \mu_{x, v}^{\prime \prime}\right) d \mu_{x, v}^{\prime}
$$

for all measurable functions $F$ on $G_{k_{v}}$. We call $d \mu_{x, v}^{\prime \prime}$ the Tamagawa measure on $G_{x k_{v}}$. Let $x \in V_{k_{v}}^{\mathrm{ss}}$. We define an invariant measure $d \tilde{\mu}_{x, v}^{\prime \prime}$ on $\widetilde{G}_{x k_{v}}=G_{x k_{v}} / \widetilde{T}_{k_{v}}$ so that

$$
\int_{g^{\prime \prime} \in G_{x} k_{v}} F^{\prime \prime}\left(g^{\prime \prime}\right) d \mu_{x, v}^{\prime \prime}=\int_{\tilde{g}^{\prime \prime} \in G_{x} k_{v} / \widetilde{T}_{k_{v}}}\left(\int_{\tilde{t}_{0} \in \widetilde{T}_{k_{v}}} F^{\prime \prime}\left(\tilde{g}^{\prime \prime} \tilde{t}_{0}\right) d \mu_{\widetilde{T}, v}\right) d \tilde{\mu}_{x, v}^{\prime \prime} .
$$

If $n$ is even then $\mathrm{GO}(x) \neq \mathrm{GO}(x)^{\circ}$. By restricting $d \mu_{x, v}^{\prime \prime}$ to $\mathrm{GO}(x)_{k_{v}}^{\circ}$, we obtain a differential form on $\mathrm{GO}(x)_{k_{v}}^{\circ}$. This differential form comes from a differential form over the global field $k$. Let $w_{v, \text { in }}^{\prime}$ be one of the alternative orbital representatives of $V_{k_{v}}^{\mathrm{ss}}$. Then $d g_{w_{v, \mathrm{i}}, v}^{\prime \prime}$ and $d \mu_{w_{v, \mathrm{i}}, v}^{\prime \prime}$ are invariant measures on $G_{w_{v, \mathrm{i}}^{\prime} k_{v}}$. So there exists a constant $c_{v, \mathrm{i}}^{\prime \prime}>0$ such that $d g_{w_{v, \mathrm{i}}^{\prime}, v}^{\prime \prime}=c_{v, \mathrm{i}}^{\prime \prime} d \mu_{w_{v, \mathrm{i}}^{\prime},}^{\prime \prime}$. We remind the reader that we shall define $d g_{w_{v, \mathrm{i}}^{\prime},}^{\prime \prime}$ for $v \in \mathfrak{M}_{\infty}$ in Part II. Let $d \widetilde{g}_{w_{v, \mathrm{i}}^{\prime}, v}^{\prime \prime}$ be the canonical measure on $\widetilde{G}_{w_{v, \mathrm{i}}^{\prime} k_{v}}$. Then $d \widetilde{g}_{w_{v, \mathrm{i}}^{\prime}, v}^{\prime \prime}$ and $d \tilde{\mu}_{w_{v, \mathrm{i}}^{\prime}, v}^{\prime \prime}$ are invariant measures on $\widetilde{G}_{w_{v, \mathrm{i}}^{\prime} k_{v}}$. So there exists a constant $\widetilde{c}_{v, \mathrm{i}}^{\prime \prime}>0$ such that $d \widetilde{g}_{w_{v, \mathrm{i}}, v}^{\prime \prime}=\widetilde{c}_{v, \mathrm{i}}^{\prime \prime} d \tilde{\mu}_{w_{v, \mathrm{i}}, v}^{\prime \prime}$. 
If $n \geq 3$ is odd then $G_{w_{v, \mathrm{i}}^{\prime} k_{v}} / \widetilde{T}_{k_{v}} \cong \mathrm{SO}\left(w_{v, \mathrm{i}}^{\prime}\right)_{k_{v}}$. So we can regard $d \widetilde{g}_{w_{v, \mathrm{i}}, v}^{\prime \prime}$, $d \widetilde{\mu}_{w_{v, \mathrm{i}}, v}^{\prime \prime}$ as measures on $\mathrm{SO}\left(w_{v, \mathrm{i}}^{\prime}\right)_{k_{v}}$. Also we can regard $\widetilde{c}_{v, \mathrm{i}}^{\prime \prime}$ as the constant which compares the canonical measure and the Tamagawa measure.

\section{Proposition 9.8.}

(1) If $v \in \mathfrak{M}_{\mathrm{f}}$ then $c_{v, \mathrm{i}}^{\prime \prime}=\left(1-q_{v}^{-1}\right)^{-1} \widetilde{c}_{v, \mathrm{i}}^{\prime \prime}$.

(2) If $v \in \mathfrak{M}_{\infty}$ then $c_{v, \mathrm{i}}^{\prime \prime}=\widetilde{c}_{v, \mathrm{i}}^{\prime \prime}$.

Proof. The statement (1) follows from (9.6). Since the Tamagawa measure on $\widetilde{T}_{k_{v}}$ is the usual measure for $v \in \mathfrak{M}_{\infty}$, the statement (2) follows.

Definition 9.9. Let $x \in V_{k}^{\mathrm{ss}}$. We define a measure $d \tilde{\mu}_{x, \mathbb{A}}^{\prime \prime}$ on $\widetilde{G}_{x \mathbb{A}}$ as follows:

$$
d \tilde{\mu}_{x, \mathbb{A}}^{\prime \prime}=\prod_{v \in \mathfrak{M}} d \tilde{\mu}_{x, v}^{\prime \prime} .
$$

It is known that $d \tilde{\mu}_{x, \mathbb{A}}^{\prime \prime}$ is well-defined since $\widetilde{G}_{x}$ is semi-simple. Since $G_{x}$ contains GL(1) as $\widetilde{T}, \prod_{v \in \mathfrak{M}_{\mathrm{f}}} \int_{G_{k} \cap K_{v}} d \mu_{x, v}^{\prime \prime}$ does not converge absolutely. So $\prod_{v \in \mathfrak{M}_{\mathrm{f}}} d \mu_{x, v}^{\prime \prime}$ is not well-defined. We put

$$
\tau\left(\widetilde{G}_{x}\right)=\left|\Delta_{k}\right|^{-\frac{\operatorname{dimsO}(x)}{2}} \int_{\widetilde{G}_{x \mathbb{A}} / \widetilde{G}_{x k}} d \tilde{\mu}_{x, \mathbb{A}}^{\prime \prime} .
$$

If $n \geq 3$ is odd then by Proposition 8.3, $d \tilde{\mu}_{x, \mathbb{A}}^{\prime \prime}$ is the Tamagawa measure on $\widetilde{G}_{x} \cong \mathrm{SO}(x)$ and $\tau\left(\widetilde{G}_{x}\right)$ is the Tamagawa number of $\mathrm{SO}(x)$. The following theorem is Theorem 4.5.1 [32, p.109].

Theorem 9.11. Suppose that $n \geq 3$ is odd. Then, for $x \in V_{k}^{\text {ss }}, \tau\left(\widetilde{G}_{x}\right)=$ 2 .

\section{§10. Unnormalized Tamagawa Number and Local Constants for Odd $n$}

Let $n \geq 3$ be odd. Suppose that $x \in V_{k}^{\mathrm{ss}}$ and $x=\alpha_{x, v} w_{v, \mathrm{i}}^{\prime}$ for $\alpha_{x, v} \in G_{k_{v}}$. We remind the reader that $G_{x}=G_{x}^{\circ}$ and $\widetilde{G}_{x}=\widetilde{G}_{x}^{\circ} \cong \mathrm{SO}(x)$ for odd $n \geq 3$. In this section we shall express the value of $\operatorname{vol}\left(\widetilde{G}_{x \mathbb{A}} / \widetilde{G}_{x k}\right)$ using $c_{v, \text { i }}^{\prime \prime}$ which we have defined just before Proposition 9.8. For $v \in \mathfrak{M}_{\infty}$, we shall compute the explicit value of $c_{v, \mathrm{i}}^{\prime \prime}$ in Part II. For $v \in \mathfrak{M}_{\mathrm{f}}$, we shall express the value of $c_{v, \text { i }}^{\prime \prime}$ using $\operatorname{vol}\left(K_{v} w_{v, \text { in }}^{\prime}\right)$ and compute the explicit value of $\operatorname{vol}\left(K_{v} w_{v, \mathrm{i}}^{\prime}\right)$ for alternative orbital representatives $w_{v \text {, in }}^{\prime}$ in $\S 11$. Thus, we obtain the explicit value of $\operatorname{vol}\left(\widetilde{G}_{x \mathbb{A}} / \widetilde{G}_{x k}\right)$ for odd $n \geq 3$.

We first prove some properties of the Tamagawa measure. 
Lemma 10.1. Let $v \in \mathfrak{M}_{\mathrm{f}}$ and $x \in V_{k_{v}}^{\mathrm{ss}}$. Then

$$
\int_{K_{v}} d \mu_{v}=\left(1-q_{v}^{-1}\right)|P(x)|_{v}^{-\frac{n+1}{2}} \operatorname{vol}\left(K_{v} x\right) \int_{\left(G_{x} k_{v} \cap K_{v}\right) \widetilde{T}_{k_{v}} / \widetilde{T}_{k_{v}}} d \tilde{\mu}_{x, v}^{\prime \prime} .
$$

Proof. Let $f_{K_{v}}$ be the characteristic function of $K_{v} \subseteq G_{k_{v}}$. If $g^{\prime} \in G_{k_{v}}$ satisfies the condition $f_{K_{v}}\left(g^{\prime} G_{x k_{v}}\right) \neq\{0\}$, then $g^{\prime} \in K_{v} G_{x k_{v}}$. Let $g^{\prime}=\kappa h$ where $\kappa \in K_{v}$ and $h \in G_{x k_{v}}$. Then, since $d \tilde{\mu}_{x, v}^{\prime \prime}$ is an invariant measure,

$$
\int_{G_{x k_{v}}} f_{K_{v}}\left(g^{\prime} h\right) d \tilde{\mu}_{x, v}^{\prime \prime}(h)=\int_{G_{x k_{v}}} f_{K_{v}}(\kappa h) d \tilde{\mu}_{x, v}^{\prime \prime}(h) .
$$

By definition, $f_{K_{v}}(\kappa h) \neq 0$ if and only if $\kappa h \in K_{v}$, which is equivalent to $h \in G_{x k_{v}} \cap K_{v}$. Since $f_{K_{v}}$ is 1 on $G_{x k_{v}} \cap K_{v}$,

$$
\int_{G_{x k_{v}}} f_{K_{v}}\left(g^{\prime} h\right) d \tilde{\mu}_{x, v}^{\prime \prime}(h)=\int_{G_{x k_{v}} \cap K_{v}} d \tilde{\mu}_{x, v}^{\prime \prime}
$$

if $g^{\prime} \in K_{v} G_{x k_{v}}$.

Therefore,

$$
\begin{aligned}
\int_{K_{v}} d \mu_{v} & =\int_{G_{x k_{v}} \cap K_{v}} d \mu_{x, v}^{\prime \prime} \int_{K_{v} G_{x k_{v}} / G_{x k_{v}}} d \mu_{x, v}^{\prime} \\
& =\int_{G_{x k_{v}} \cap K_{v}} d \mu_{x, v}^{\prime \prime} \int_{K_{v} x}|P(y)|_{v}^{-\frac{n+1}{2}} d y .
\end{aligned}
$$

Since $P(y)=P(x)$ for $y \in K_{v} x$,

$$
\int_{K_{v} x}|P(y)|_{v}^{-\frac{n+1}{2}} d y=|P(x)|_{v}^{-\frac{n+1}{2}} \int_{K_{v} x} d y=|P(x)|_{v}^{-\frac{n+1}{2}} \operatorname{vol}_{v}\left(K_{v} x\right) .
$$

The set $G_{x k_{v}} \cap K_{v}$ surjects to $\left(G_{x k_{v}} \cap K_{v}\right) \widetilde{T}_{k_{v}} / \widetilde{T}_{k_{v}}$. If

$$
\left(t_{0}, g_{1}\right),\left(s_{0}, h_{1}\right) \in G_{x k_{v}} \cap K_{v}
$$

and $\left(t_{0}, g_{1}\right)\left(\tilde{t}_{0}^{-2}, \tilde{t}_{0} I_{n}\right)=\left(s_{0}, h_{1}\right)$ for some $\tilde{t}_{0} \in \widetilde{T}_{k_{v}}$, then

$$
\left(\tilde{t}_{0}^{-2}, \tilde{t}_{0} I_{n}\right)=\left(t_{0}, g_{1}\right)^{-1}\left(s_{0}, h_{1}\right) \in \widetilde{T}_{k_{v}} \cap K_{v},
$$

which implies that $\tilde{t}_{0} \in \mathcal{O}_{v}^{\times}$.

If $d \mu_{\widetilde{T}, v}$ is the Tamagawa measure on $\widetilde{T}_{k_{v}} \cong \mathrm{GL}(1)_{k_{v}}, \int_{\widetilde{T}_{k_{v}} \cap K_{v}} d \mu_{\widetilde{T}, v}=$ $\left(1-q_{v}^{-1}\right)$. So

$$
\begin{aligned}
\int_{G_{x k_{v}} \cap K_{v}} d \mu_{x, v}^{\prime \prime} & =\int_{\left(G_{x k_{v}} \cap K_{v}\right) \widetilde{T}_{k_{v}} / \widetilde{T}_{k_{v}}}\left(\int_{\widetilde{T}_{k_{v}} \cap K_{v}} d \mu_{\widetilde{T}, v}\right) d \tilde{\mu}_{x, v}^{\prime \prime} \\
& =\left(1-q_{v}^{-1}\right) \int_{\left(G_{x k_{v}} \cap K_{v}\right) \widetilde{T}_{k_{v}} / \widetilde{T}_{k_{v}}} d \tilde{\mu}_{x, v}^{\prime \prime} .
\end{aligned}
$$


Let $v \in \mathfrak{M}$ be an arbitrary place. Suppose that $g_{0} \in G_{k_{v}}$ and $x=g_{0} x^{\prime} \in$ $V_{k_{v}}^{\text {ss }}$. We define $\varphi_{g_{0}}: G_{x k_{v}} \rightarrow G_{x^{\prime} k_{v}}$, etc., similarly as in (8.1).

Proposition 10.2. On $G_{x k_{v}}, d \mu_{x, v}^{\prime \prime}=\varphi_{g_{0}}^{*}\left(d \mu_{x^{\prime}, v}^{\prime \prime}\right)$. On $\widetilde{G}_{x k_{v}}, d \tilde{\mu}_{x, v}^{\prime \prime}=$ $\varphi_{g_{0}}^{*}\left(d \tilde{\mu}_{x^{\prime}, v}^{\prime \prime}\right)$.

Proof. Note that $d \mu_{v}=d \mu_{x, v}^{\prime} d \mu_{x, v}^{\prime \prime}=d \mu_{x^{\prime}, v}^{\prime} d \mu_{x^{\prime}, v}^{\prime \prime}$. Since $\varphi_{g_{0}}^{*}\left(d \mu_{v}\right)=d \mu_{v}$,

$$
d \mu_{v}=\varphi_{g_{0}}^{*}\left(d \mu_{x^{\prime}, v}^{\prime}\right) \varphi_{g_{0}}^{*}\left(d \mu_{x^{\prime}, v}^{\prime \prime}\right) .
$$

So we only have to prove that $d \mu_{x, v}^{\prime}=\varphi_{g_{0}}^{*}\left(d \mu_{x^{\prime}, v}^{\prime}\right)$.

If $F^{\prime}$ is a measurable function on $V_{k_{v}}^{\text {ss }}$, then $F^{\prime}(* x)$ is a measurable function on $G_{k_{v}} / G_{x k_{v}}$. We denote this function by $\bar{F}^{\prime}$. Then

$$
\begin{aligned}
\int_{h_{x} \in G_{k_{v}} / G_{x} k_{v}} \bar{F}^{\prime}\left(h_{x}\right) \varphi_{g_{0}}^{*}\left(d \mu_{x^{\prime}, v}^{\prime}\right) & =\int_{h_{x^{\prime}} \in G_{k_{v}} / G_{x^{\prime} k_{v}}} \bar{F}^{\prime} \circ \varphi_{g_{0}}^{-1}\left(h_{x^{\prime}}\right) d \mu_{x^{\prime}, v}^{\prime} \\
& =\int_{h_{x^{\prime}} \in G_{k_{v}} / G_{x^{\prime} k_{v}}}^{\prime} F^{\prime}\left(g_{0} h_{x^{\prime}} g_{0}^{-1} x\right) d \mu_{x^{\prime}, v}^{\prime} \\
& =\int_{h_{x^{\prime}} \in G_{k_{v}} / G_{x^{\prime} k_{v}}} F^{\prime}\left(g_{0} h_{x^{\prime}} x^{\prime}\right) d \mu_{x^{\prime}, v}^{\prime}
\end{aligned}
$$

Since $d \mu_{x^{\prime}, v}^{\prime}$ is left $G_{k_{v}}$-invariant, (10.3) is equal to

$$
\begin{aligned}
\int_{h_{x^{\prime}} \in G_{k_{v}} / G_{x^{\prime} k_{v}}} F^{\prime}\left(h_{x^{\prime}} x^{\prime}\right) d \mu_{x^{\prime}, v}^{\prime} & =\int_{y \in G_{k_{v}} x^{\prime}} F^{\prime}(y)|P(y)|_{v}^{-\frac{n+1}{2}} d y \\
& =\int_{y \in G_{k_{v}} x} F^{\prime}(y)|P(y)|^{-\frac{n+1}{2}} d y \\
& =\int_{h_{x} \in G_{k_{v}} / G_{x k_{v}}} \bar{F}^{\prime}\left(h_{x}\right) d \mu_{x, v}^{\prime} .
\end{aligned}
$$

Therefore, $\varphi_{g_{0}}^{*}\left(d \mu_{x^{\prime}, v}^{\prime}\right)=d \mu_{x, v}^{\prime}$. So $\varphi_{g_{0}}^{*}\left(d \mu_{x^{\prime}, v}^{\prime \prime}\right)=d \mu_{x, v}^{\prime \prime}$

Let $d \mu_{\widetilde{T}, v}$ be the Tamagawa measure on $\widetilde{T}_{k_{v}}$ as before. Then

$$
d \mu_{x, v}^{\prime}=d \tilde{\mu}_{x, v}^{\prime} d \mu_{\widetilde{T}, v}, \quad d \mu_{x^{\prime}, v}^{\prime \prime}=d \tilde{\mu}_{x^{\prime}, v}^{\prime \prime} d \mu_{\widetilde{T}, v} .
$$

Since

$$
\begin{aligned}
& d \mu_{x, v}^{\prime \prime}=\varphi_{g_{0}}^{*}\left(d \mu_{x^{\prime}, v}^{\prime \prime}\right)=\varphi_{g_{0}}^{*}\left(d \tilde{\mu}_{x^{\prime}, v}^{\prime \prime} d \mu_{\widetilde{T}, v}\right)=\varphi_{g_{0}}^{*}\left(d \tilde{\mu}_{x^{\prime}, v}^{\prime \prime}\right) d \mu_{\widetilde{T}, v}, \\
& d \tilde{\mu}_{x, v}^{\prime \prime}=\varphi_{g_{0}}^{*}\left(d \tilde{\mu}_{x^{\prime}, v}^{\prime \prime}\right) .
\end{aligned}
$$

Suppose that $x \in V_{k_{v}}^{\mathrm{ss}}$. Let $\dot{\mathrm{i}}_{v}(x)$ be an index such that $G_{k_{v}} x=G_{k_{v}} w_{v, \dot{\mathrm{i}}_{v}(x)}$. 
Proposition 10.4. Suppose that $n \geq 3$ is odd and $x \in V_{k}^{\mathrm{ss}}$. Then

$$
\operatorname{vol}\left(\widetilde{G}_{x \mathbb{A}} / \widetilde{G}_{x k}\right)=2\left|\Delta_{k}\right|^{\frac{\operatorname{dimSO}(x)}{2}} \prod_{v \in \mathfrak{M}} \widetilde{c}_{v, \dot{\mathrm{i}}_{v}(x)}^{\prime \prime} .
$$

Proof. By Proposition 10.2,

$$
\begin{aligned}
d \widetilde{g}_{x, v}^{\prime \prime} & =\varphi_{g_{x}}^{*}\left(d \widetilde{g}_{v,,_{i}(x)}^{\prime \prime}\right)=\varphi_{g_{x}}^{*}\left(\widetilde{c}_{v, \dot{\mathrm{i}}_{v}(x)}^{\prime \prime} d \widetilde{\mu}_{w_{v, \mathrm{i}_{v}(x)}^{\prime \prime}, v}^{\prime \prime}\right) \\
& =\widetilde{c}_{v, \dot{\mathrm{i}}_{v}(x)}^{\prime \prime} \varphi_{g_{x}}^{*}\left(d \widetilde{\mu}_{w_{v, \mathrm{i}_{v}(x)}^{\prime \prime}, v}^{\prime \prime}\right)=\widetilde{c}_{v, \dot{\mathrm{i}}_{v}(x)}^{\prime \prime} d \widetilde{\mu}_{x, v}^{\prime \prime} .
\end{aligned}
$$

Therefore, $d \widetilde{g}_{x, \mathbb{A}}^{\prime \prime}=\prod_{v \in \mathfrak{M}} d \widetilde{g}_{x, v}^{\prime \prime}=\prod_{v \in \mathfrak{M}} \widetilde{c}_{v,,_{v}(x)}^{\prime \prime} d \widetilde{\mu}_{x, \mathbb{A}}^{\prime \prime}$. By Theorem 9.11,

$$
\begin{aligned}
\operatorname{vol}\left(\widetilde{G}_{x \mathbb{A}} / \widetilde{G}_{x k}\right) & =\int_{\widetilde{G}_{x \mathbb{A}} / \widetilde{G}_{x k}} d \widetilde{g}_{x, \mathbb{A}}^{\prime \prime}=\prod_{v \in \mathfrak{M}} \widetilde{c}_{v, \dot{\mathrm{i}}_{v}(x)}^{\prime \prime} \int_{\widetilde{G}_{x \mathbb{A}} / \widetilde{G}_{x k}} d \widetilde{\mu}_{x, \mathbb{A}}^{\prime \prime} \\
& =2\left|\Delta_{k}\right|^{\frac{\operatorname{dimSO}(x)}{2}} \prod_{v \in \mathfrak{M}} \widetilde{c}_{v, \dot{\mathrm{i}}_{v}(x)}^{\prime \prime} .
\end{aligned}
$$

\section{$\S 11 . \quad$ The Value of the Constant $\widetilde{c}_{v, \text { i }}^{\prime}$ at Finite Places}

Throughout this section we assume that $n \geq 3$ is odd and $v \in \mathfrak{M}_{\mathrm{f}}$. In this section we compute the value of $\widetilde{c}_{v, \mathrm{i}}^{\prime \prime}$. We first express $\widetilde{c}_{v, \mathrm{i}}^{\prime \prime}$ in terms of $\operatorname{vol}\left(K_{v} w_{v, \dot{\mathrm{i}}}\right)$.

Lemma 11.1. Let $v \in \mathfrak{M}_{\mathrm{f}}$ and $w_{v, \text { i }}$ be a standard orbital representative for $G_{k_{v}} \backslash V_{k_{v}}^{\mathrm{ss}}$. Then

$$
\widetilde{c}_{v, \mathrm{i}}^{\prime \prime}=\left|P\left(w_{v, \text { i }}\right)\right|_{v}^{-\frac{n+1}{2}} \operatorname{vol}\left(K_{v} w_{v, \mathrm{i}}\right) \prod_{j=1}^{n}\left(1-q_{v}^{-j}\right)^{-1} .
$$

Proof. By the definition of $d \widetilde{g}_{w_{v, \mathrm{i}}, v}^{\prime \prime}$,

$$
\int_{\left(G_{w_{v}, \mathrm{i}} k_{v} \cap K_{v}\right) \widetilde{T}_{k_{v}} / \widetilde{T}_{k_{v}}} d \widetilde{g}_{w_{v, \mathrm{i}}, v}^{\prime \prime}=1 .
$$

By the definition of $\widetilde{c}_{v, \text { in }}^{\prime \prime}$,

$$
\begin{aligned}
\widetilde{c}_{v, \mathrm{i}}^{\prime \prime} & =\int_{\left(G_{w_{v, \mathrm{i}} k_{v}} \cap K_{v}\right) \widetilde{T}_{k_{v}} / \widetilde{T}_{k_{v}}} d \widetilde{g}_{w_{v, \mathrm{i}}, v}^{\prime \prime}\left(\int_{\left(G_{w_{v, \mathrm{i}} k_{v}} \cap K_{v}\right) \widetilde{T}_{k_{v}} / \widetilde{T}_{k_{v}}} d \widetilde{\mu}_{w_{v, \mathrm{i}}, v}^{\prime \prime}\right)^{-1} \\
& =\left(\int_{\left(G_{w_{v, \mathrm{i}} k_{v}} \cap K_{v}\right) \widetilde{T}_{k_{v}} / \widetilde{T}_{k_{v}}} d \widetilde{\mu}_{w_{v, \mathrm{i}}, v}^{\prime \prime}\right)^{-1} .
\end{aligned}
$$


Using Theorem 9.3 and Lemma 10.1,

$$
\begin{aligned}
\int_{\left(G_{w_{v, \mathrm{i}} k_{v}} \cap K_{v}\right) \widetilde{T}_{k_{v}} / \widetilde{T}_{k_{v}}} d \widetilde{\mu}_{w_{v, \mathrm{i}}, v}^{\prime \prime} & =\left(1-q_{v}^{-1}\right)^{-1}\left|P\left(w_{v, \mathrm{i}}\right)\right|_{v^{\frac{n+1}{2}}}\left(\operatorname{vol}\left(K_{v} w_{v, \mathrm{i}}\right)\right)^{-1} \int_{K_{v}} d \mu_{v} \\
& =\left|P\left(w_{v, \mathrm{i}}\right)\right|_{v^{\frac{n+1}{2}}}\left(\operatorname{vol}\left(K_{v} w_{v, \mathrm{i}}\right)\right)^{-1} \prod_{j=1}^{n}\left(1-q_{v}^{-j}\right) .
\end{aligned}
$$

Therefore,

$$
\widetilde{c}_{v, \mathrm{i}}^{\prime \prime}=\left|P\left(w_{v, \mathrm{i}}\right)\right|_{v}^{-\frac{n+1}{2}} \operatorname{vol}\left(K_{v} w_{v, \mathrm{i}}\right) \prod_{j=1}^{n}\left(1-q_{v}^{-j}\right)^{-1} .
$$

By Lemma 11.1, we only have to compute the value of $\operatorname{vol}\left(K_{v} w_{v, \text { in }}\right)$ in order to determine the value of the constant $\widetilde{c}_{v, \mathrm{i}}^{\prime \prime}$. We first define some notations used in the computation of the value of $\operatorname{vol}\left(K_{v} w_{v, \mathrm{i}}\right)$. Let $m_{v}=\operatorname{ord}_{v} 2$ and $\mathbb{F}_{v}=\mathcal{O}_{v} / \mathfrak{p}_{v}$. Note that $\mathbb{F}_{v} \cong \mathbb{F}_{q_{v}}$ where $q_{v}=\sharp\left(\mathcal{O}_{v} / \pi_{v} \mathcal{O}_{v}\right)$. If there is no confusion, for $c \in \mathcal{O}_{v}$, we denote the element $c \bmod \pi_{v}$ of $\mathbb{F}_{v}$ also by $c$ by abuse of notation. We denote the set of squares of $\mathbb{F}_{v}^{\times}$by $\left(\mathbb{F}_{v}^{\times}\right)^{2}$. It is known that $\left[\mathbb{F}_{v}^{\times}:\left(\mathbb{F}_{v}^{\times}\right)^{2}\right]=2$ if $v \notin \mathfrak{M}_{\mathrm{dy}}$, and $\left(\mathbb{F}_{v}^{\times}\right)^{2}=\mathbb{F}_{v}^{\times}$if $v \in \mathfrak{M}_{\mathrm{dy}}$. We put

$$
\delta_{v}= \begin{cases}1 & v \notin \mathfrak{M}_{\mathrm{dy}}, \\ 0 & v \in \mathfrak{M}_{\mathrm{dy}},\end{cases}
$$

i.e., $2^{\delta_{v}}=\left[\mathbb{F}_{v}^{\times}:\left(\mathbb{F}_{v}^{\times}\right)^{2}\right]$ and $\sharp\left(\mathbb{F}_{v}^{\times}\right)^{2}=2^{-\delta_{v}} \sharp \mathbb{F}_{v}^{\times}$.

If $x=\left(x_{i j}\right), y=\left(y_{i j}\right) \in V_{\mathcal{O}_{v}}$ and $d \in \mathbb{Z}_{>0}$, then we use the notation $x \equiv y$ $\bmod \pi_{v}^{d}$, etc., if $x_{i j} \equiv y_{i j} \bmod \pi_{v}^{d}$ for all $i, j$. Note that we use the coordinate system (3.2), and regard 2's in the diagonal entries of (3.3) as formal coefficients.

Let $a_{0}, b_{0} \in \mathcal{O}_{v}^{\times}$be as in (4.2). Then a root of $z^{2}+a_{0} z+b_{0} \in \mathbb{F}_{v}[z]$ generates the unique unramified quadratic extension $L_{v}$ of $\mathbb{F}_{v}$. Let $\alpha_{0}, \beta_{0} \in L_{v}$ be the elements such that

$$
z^{2}+a_{0} z+b_{0}=\left(z+\alpha_{0}\right)\left(z+\beta_{0}\right) .
$$

We denote the conjugate of $\eta \in L_{v}$ by $\bar{\eta}$, and the norm map $L_{v}^{\times} \ni \eta \mapsto \eta \bar{\eta} \in \mathbb{F}_{v}^{\times}$ by $N_{L_{v} / \mathbb{F}_{v}}$. It is known that $N_{L_{v} / \mathbb{F}_{v}}\left(L_{v}^{\times}\right)=\mathbb{F}_{v}^{\times}$.

Before computing the value of $\operatorname{vol}\left(K_{v} w_{v \text {,i }}\right)$, we review some facts concerning orders of general orthogonal groups over $\mathbb{F}_{v}$. Since we shall use similar computations in Part III, we consider all $n$ (which means that we do not assume that $n$ is odd). 
We define an $n$-ary quadratic form $Q_{n, \text { sp }}$ over $\mathbb{F}_{v}$ as follows:

$$
Q_{n, \mathrm{sp}}[X]= \begin{cases}X_{1} X_{2}+\cdots+X_{n-2} X_{n-1}+X_{n}^{2} & n \geq 3 \text { odd } \\ X_{1} X_{2}+\cdots+X_{n-1} X_{n} & n \geq 4 \text { even }\end{cases}
$$

where $X=\left(X_{1}, \ldots, X_{n}\right)$ and $X_{1}, \ldots, X_{n}$ are variables. Similarly, for odd $n \geq 3$, we define an $(n-1)$-ary quadratic form $Q_{n-1, \text { in }}$ over $\mathbb{F}_{v}$ as follows:

$$
Q_{n-1, \mathrm{in}}[X]=X_{1} X_{2}+\cdots+X_{n-4} X_{n-3}+X_{n-2}^{2}+a_{0} X_{n-2} X_{n-1}+b_{0} X_{n-1}^{2}
$$

where $X=\left(X_{1}, \ldots, X_{n-1}\right)$ and $X_{1}, \ldots, X_{n-1}$ are variables.

The following lemma is known (see [12, pp.146-147]).

Lemma 11.2. We have

$$
\sharp \mathrm{O}\left(Q_{n, \mathrm{sp}}\right)_{\mathbb{F}_{v}}= \begin{cases}2^{\delta_{v}} q_{v}^{\frac{n(n-1)}{2}} \prod_{i=1}^{\frac{n-1}{2}}\left(1-q_{v}^{-2 i}\right) & n \text { odd, } \\ 2 q_{v}^{\frac{n(n-1)}{2}}\left(1+q_{v}^{-\frac{n}{2}}\right)^{-1} \prod_{i=1}^{\frac{n}{2}}\left(1-q_{v}^{-2 i}\right) & n \text { even } .\end{cases}
$$

If $n \geq 3$ is odd then

$$
\sharp \mathrm{O}\left(Q_{n-1, \mathrm{in}}\right)_{\mathbb{F}_{v}}=2 q_{v}^{\frac{(n-1)(n-2)}{2}}\left(1-q_{v}^{-\frac{n-1}{2}}\right)^{-1} \prod_{i=1}^{\frac{n-1}{2}}\left(1-q_{v}^{-2 i}\right) .
$$

We now compute the values of the orders of $\operatorname{GO}\left(Q_{n-1, \text { in }}\right)_{\mathbb{F}_{v}}, \operatorname{GO}\left(Q_{n, \text { sp }}\right)_{\mathbb{F}_{v}}$ using Lemma 11.2. For each general orthogonal group, we denote its multiplicator by $\gamma(*)$ similarly as in previous sections.

We first consider $\operatorname{GO}\left(Q_{n, \mathrm{sp}}\right)_{\mathbb{F}_{v}}$ for even $n$. Let $\gamma \in \mathbb{F}_{v}^{\times}$and

$$
Y=\left(Y_{1}, Y_{2}, \cdots, Y_{n-1}, Y_{n}\right)=\left(X_{1}, \gamma X_{2}, X_{3}, \gamma X_{4}, \cdots, X_{n-1}, \gamma X_{n}\right) .
$$

Then $Q_{n, \mathrm{sp}}[Y]=\gamma Q_{n, \mathrm{sp}}[X]$. So the multiplicator $\gamma: \operatorname{GO}\left(Q_{n, \mathrm{sp}}\right)_{\mathbb{F}_{v}} \ni g \mapsto$ $\gamma(g) \in \mathrm{GL}(1)_{\mathbb{F}_{v}}$ is a surjective group homomorphism. Since the kernel of $\gamma$ is $\mathrm{O}\left(Q_{n, \mathrm{sp}}\right)_{\mathbb{F}_{v}}$, the sequence

$$
1 \rightarrow \mathrm{O}\left(Q_{n, \mathrm{sp}}\right)_{\mathbb{F}_{v}} \rightarrow \mathrm{GO}\left(Q_{n, \mathrm{sp}}\right)_{\mathbb{F}_{v}} \rightarrow \mathrm{GL}(1)_{\mathbb{F}_{v}} \rightarrow 1
$$

is exact. Thus,

$$
\sharp \mathrm{GO}\left(Q_{n, \mathrm{sp}}\right)_{\mathbb{F}_{v}}=\sharp \mathrm{GL}(1)_{\mathbb{F}_{v}} \times \sharp \mathrm{O}\left(Q_{n, \mathrm{sp}}\right)_{\mathbb{F}_{v}}=\sharp \mathbb{F}_{v}^{\times} \times \sharp \mathrm{O}\left(Q_{n-1, \mathrm{sp}}\right)_{\mathbb{F}_{v}} .
$$


We next consider $\operatorname{GO}\left(Q_{n-1, \text { in }}\right)_{\mathbb{F}_{v}}$ for odd $n$. We define binary quadratic forms $Q_{\mathrm{sp}}^{0}$ and $Q_{\mathrm{in}}^{0}$ over $\mathbb{F}_{v}$ as follows:

$$
Q_{\mathrm{sp}}^{0}[X]=X_{n-2} X_{n-1}, \quad Q_{\mathrm{in}}^{0}[X]=X_{n-2}^{2}+a_{0} X_{n-2} X_{n-1}+b_{0} X_{n-1}^{2},
$$

where $X=\left(X_{n-2}, X_{n-1}\right)$ and $X_{n-2}, X_{n-1}$ are variables. Let $\gamma \in \mathbb{F}_{v}^{\times}$. Since $L_{v} / \mathbb{F}_{v}$ is a finite extension of finite fields, $N_{L_{v} / \mathbb{F}_{v}}\left(L_{v}^{\times}\right)=\mathbb{F}_{v}^{\times}$. So there exists $\eta \in L_{v}^{\times}$such that $\gamma=\eta \bar{\eta}$. We put

$$
h_{v}=\left(\begin{array}{cc}
1 & 1 \\
\alpha_{0} & \beta_{0}
\end{array}\right), a(\eta)=\left(\begin{array}{cc}
\eta & 0 \\
0 & \bar{\eta}
\end{array}\right) \in \mathrm{GL}(2)_{L_{v}} .
$$

Note that $h_{v} a(\eta) h_{v}^{-1} \in \mathrm{GL}(2)_{\mathbb{F}_{v}}$. Since $h_{v}^{-1} Q_{\mathrm{in}}^{0}=Q_{\mathrm{sp}}^{0}$ and $a(\eta) Q_{\mathrm{sp}}^{0}=\gamma Q_{\mathrm{sp}}^{0}$,

$$
h_{v} a(\eta) h_{v}^{-1} Q_{\mathrm{in}}^{0}=\gamma Q_{\mathrm{in}}^{0} .
$$

Let

$$
\begin{aligned}
\left(Y_{1}, Y_{2}, \cdots, Y_{n-4}, Y_{n-3}\right) & =\left(X_{1}, \gamma X_{2}, X_{3}, \gamma X_{4}, \cdots, X_{n-4}, \gamma X_{n-3}\right), \\
\left(Y_{n-2}, Y_{n-1}\right) & =\left(X_{n-2}, X_{n-1}\right) h_{v} a(\eta) h_{v}^{-1} .
\end{aligned}
$$

Then

$$
\begin{aligned}
Q_{n-1, \text { in }}[Y] & =\gamma\left(X_{1} X_{2}+\cdots+X_{n-4} X_{n-3}+X_{n-2}^{2}+a_{0} X_{n-2} X_{n-1}+b_{0} X_{n-1}^{2}\right) \\
& =\gamma Q_{n-1, \text { in }}[X] .
\end{aligned}
$$

So the multiplicator $\operatorname{GO}\left(Q_{n-1, \text { in }}\right)_{\mathbb{F}_{v}} \ni g \mapsto \gamma(g) \in \mathrm{GL}(1)_{\mathbb{F}_{v}}$ is a surjective group homomorphism. Since the kernel of the multiplicator is $\mathrm{O}\left(Q_{n-1, \text { in }}\right)_{\mathbb{F}_{v}}$, the sequence

$$
1 \rightarrow \mathrm{O}\left(Q_{n-1, \text { in }}\right)_{\mathbb{F}_{v}} \rightarrow \mathrm{GO}\left(Q_{n-1, \text { in }}\right)_{\mathbb{F}_{v}} \rightarrow \mathrm{GL}(1)_{\mathbb{F}_{v}} \rightarrow 1
$$

is exact. Thus,

$$
\sharp \mathrm{GO}\left(Q_{n-1, \text { in }}\right)_{\mathbb{F}_{v}}=\sharp \mathrm{GL}(1)_{\mathbb{F}_{v}} \times \sharp \mathrm{O}\left(Q_{n-1, \text { in }}\right)_{\mathbb{F}_{v}}=\sharp \mathbb{F}_{v}^{\times} \times \sharp \mathrm{O}\left(Q_{n-1, \text { in }}\right)_{\mathbb{F}_{v}} .
$$

We next consider $\operatorname{GO}\left(Q_{n, \mathrm{sp}}\right)_{\mathbb{F}_{v}}$ for odd $n$. Since $n$ is odd,

$$
\mathrm{GO}\left(Q_{n, \mathrm{sp}}\right)_{\mathbb{F}_{v}} \cong \mathrm{SO}\left(Q_{n, \mathrm{sp}}\right)_{\mathbb{F}_{v}} \times\left\{\tilde{t}_{0} I_{n} \mid \tilde{t}_{0} \in \mathbb{F}_{v}^{\times}\right\} .
$$

The multiplicator $\gamma(g)$ is equal to 1 for $g \in \operatorname{SO}\left(Q_{n, \mathrm{sp}}\right)_{\mathbb{F}_{v}}$ and $\gamma\left(\tilde{t}_{0} I_{n}\right)=\tilde{t}_{0}^{2}$. Therefore, the image of the multiplicator is $\left(\mathbb{F}_{v}^{\times}\right)^{2}$ and

$$
1 \rightarrow \mathrm{O}\left(Q_{n, \mathrm{sp}}\right)_{\mathbb{F}_{v}} \rightarrow \mathrm{GO}\left(Q_{n, \mathrm{sp}}\right)_{\mathbb{F}_{v}} \rightarrow\left(\mathbb{F}_{v}^{\times}\right)^{2} \rightarrow 1
$$


is an exact sequence. Thus,

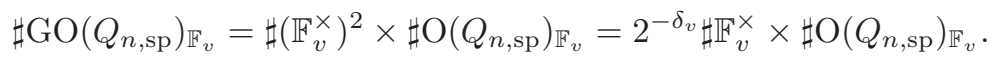

Therefore, we arrive at the following lemma.

Lemma 11.3. We have

$$
\sharp \mathrm{GO}\left(Q_{n, \mathrm{sp}}\right)_{\mathbb{F}_{v}}= \begin{cases}q^{\frac{n(n-1)}{2}+1}\left(1-q_{v}^{-1}\right) \prod_{i=1}^{\frac{n-1}{2}}\left(1-q_{v}^{-2 i}\right) & n \text { odd, } \\ 2 q^{\frac{n(n-1)}{2}+1}\left(1-q_{v}^{-1}\right)\left(1+q_{v}^{-\frac{n}{2}}\right)^{-1} \prod_{i=1}^{\frac{n}{2}}\left(1-q_{v}^{-2 i}\right) & n \text { even. }\end{cases}
$$

If $n \geq 3$ is odd, then

$$
\sharp \mathrm{GO}\left(Q_{n-1, \mathrm{in}}\right)_{\mathbb{F}_{v}}=2 q_{v}^{\frac{(n-1)(n-2)}{2}+1}\left(1-q_{v}^{-1}\right)\left(1-q_{v}^{-\frac{n-1}{2}}\right)^{-1} \prod_{i=1}^{\frac{n-1}{2}}\left(1-q_{v}^{-2 i}\right) .
$$

Let $Q$ be a binary quadratic form defined by

$$
Q[X]=c_{1} X_{1}^{2}+c_{2} X_{1} X_{2}+c_{3} X_{2}^{2} \quad\left(c_{1}, c_{2}, c_{3} \in \mathcal{O}_{v}\right)
$$

for $X=\left(X_{1}, X_{2}\right)$ where $X_{1}, X_{2}$ are variables. In the process of computing the value of $\operatorname{vol}\left(K_{v} w_{v, \text { i }}\right)$, we shall use the following lemma.

Lemma 11.4. Suppose that $Q[X] \equiv Q_{\mathrm{sp}}^{0}[X] \bmod \pi_{v}$. If $\gamma \in \mathcal{O}_{v}^{\times}$, there exists $g \in \mathrm{GL}(2)_{\mathcal{O}_{v}}$ such that $g Q[X]=\gamma Q_{\mathrm{sp}}^{0}[X]$. Suppose that $Q[X] \equiv Q_{\mathrm{in}}^{0}[X]$ $\bmod \pi_{v}$. If $\gamma \in \mathcal{O}_{v}^{\times}$, there exists $g \in \mathrm{GL}(2)_{\mathcal{O}_{v}}$ such that $g Q[X]=\gamma Q_{\text {in }}^{0}[X]$.

Proof. Since the argument is similar for $Q_{\mathrm{sp}}^{0}[X]$, we only consider $Q_{\mathrm{in}}^{0}[X]$ $=X_{1}^{2}+a_{0} X_{1} X_{2}+b_{0} X_{2}^{2}$. Suppose that $Q[X] \equiv Q_{\mathrm{in}}^{0}[X] \bmod \pi_{v}$. We put

$$
Y=X\left(\begin{array}{cc}
z_{1} z_{2} \\
z_{3} & 1
\end{array}\right), \quad Q[Y]-Q_{\mathrm{in}}^{0}[X] \equiv f_{1} X_{1}^{2}+f_{2} X_{1} X_{2}+f_{3} X_{2}^{2} \quad \bmod \pi_{v} .
$$

Then

$$
\begin{aligned}
& f_{1}=z_{1}^{2}+a_{0} z_{1} z_{2}+b_{0} z_{2}^{2}-1, \\
& f_{2}=2 z_{1} z_{3}+a_{0} z_{1}+a_{0} z_{2} z_{3}+2 b_{0} z_{2}-a_{0}, \\
& f_{3}=z_{3}^{2}+a_{0} z_{3} .
\end{aligned}
$$


By easy computations,

$$
J\left(z_{1}, z_{2}, z_{3}\right) \stackrel{\text { def }}{=}\left(\frac{\partial f_{i}}{\partial z_{j}}\right)_{1 \leq i, j \leq 3}=\left(\begin{array}{ccc}
2 z_{1}+a_{0} z_{2} & a_{0} z_{1}+2 b_{0} z_{2} & 0 \\
2 z_{3}+a_{0} & a_{0} z_{3}+2 b_{0} & 2 z_{1}+a_{0} z_{2} \\
0 & 0 & 2 z_{3}+a_{0}
\end{array}\right)
$$

and $\operatorname{det} J(1,0,0)=-a_{0}\left(a_{0}^{2}-4 b_{0}\right) \in \mathbb{F}_{v}^{\times}$. So, by using Hensel's lemma, there exists $g_{1} \in \mathrm{M}(2,2)_{\mathcal{O}_{v}}$ such that

$$
g_{1} \equiv\left(\begin{array}{ll}
1 & 0 \\
0 & 1
\end{array}\right) \quad \bmod \pi_{v}, \quad g_{1} Q[X]=Q_{\mathrm{in}}^{0}[X] .
$$

Since $g \equiv I_{2} \bmod \pi_{v}, g_{1} \in \mathrm{GL}(2)_{\mathcal{O}_{v}}$.

Let $\gamma \in \mathcal{O}_{v}^{\times}$. By the same argument as in the proof of the surjectivity of the multiplicator

$$
\mathrm{GO}\left(Q_{\text {in }}^{0}\right)_{\mathbb{F}_{v}} \ni g \mapsto \gamma(g) \in \mathrm{GL}(1)_{\mathbb{F}_{v}},
$$

there exists $g_{2} \in \mathrm{GL}(2)_{\mathcal{O}_{v}}$ such that $g_{2} Q_{\text {in }}^{0}[X]=\gamma Q_{\text {in }}^{0}[X]$. Thus, for $g=g_{2} g_{1}$, $g_{2} g_{1} Q[X]=\gamma Q_{\text {in }}^{0}[X]$.

Now we return to the case where $n \geq 3$ is odd, and compute the value of $\operatorname{vol}\left(K_{v} w_{v, \mathrm{i}}\right)$. We first consider $\operatorname{vol}\left(K_{v} w_{v, \mathrm{sp}}\right)$. We define a subset $\mathcal{D}_{v, \mathrm{sp}}$ of $V_{\mathcal{O}_{v}}$ as follows:

$$
\mathcal{D}_{v, \mathrm{sp}}=\left\{y \in V_{\mathcal{O}_{v}} \mid y \equiv w_{v, \mathrm{sp}} \bmod \pi_{v}\right\}
$$

Lemma 11.6. $K_{v} w_{v, \mathrm{sp}}=K_{v} \mathcal{D}_{v, \mathrm{sp}}$.

Proof. Using block matrices, we can express $Y \in \mathcal{D}_{v, \text { sp }}$ as follows:

$$
Y=\left(\begin{array}{cc}
Y_{1} & Y_{2} \\
{ }^{t} Y_{2} & Y_{3}
\end{array}\right)
$$

where $Y_{1} \in \mathrm{M}(2,2)_{\mathcal{O}_{v}}, Y_{2} \in \mathrm{M}(2, n-2)_{\mathcal{O}_{v}}, Y_{3} \in \mathrm{M}(n-2, n-2)_{\mathcal{O}_{v}}$. Since $Y_{1} \equiv\left(\begin{array}{ll}0 & 1 \\ 1 & 0\end{array}\right) \bmod \pi_{v}, Y_{1} \in \mathrm{GL}(2)_{\mathcal{O}_{v}}$. Therefore,

$$
g_{1}=\left(\begin{array}{cc}
I_{2} & 0_{2, n-2} \\
-{ }^{t} Y_{2} Y_{1}^{-1} & I_{n-2}
\end{array}\right) \in K_{v}, \quad g_{1} Y^{t} g_{1}=\left(\begin{array}{cc}
Y_{1} & 0_{2, n-2} \\
0_{n-2,2} & Y_{3}-{ }^{t} Y_{2} Y_{1}^{-1} Y_{2}
\end{array}\right)
$$

Since $Y \in \mathcal{D}_{v, \mathrm{sp}}, Y_{2} \equiv 0 \bmod \pi_{v}$. Since $Y_{3}-{ }^{t} Y_{2} Y_{1}^{-1} Y_{2} \equiv Y_{3} \bmod \pi_{v}$, $g_{1} Y^{t} g_{1} \in \mathcal{D}_{v, \mathrm{sp}}$. So, by applying an element of $K_{v}, Y$ can be made into the 
following form:

$$
Y=\left(\begin{array}{llll}
Y_{1} & & & \\
& \ddots & & \\
& & Y_{\frac{n-1}{2}} & \\
& & & 2 Y_{\frac{n+1}{2}}
\end{array}\right)
$$

where $Y_{1}, \cdots, Y_{\frac{n-1}{2}} \in \mathrm{M}(2,2)_{\mathcal{O}_{v}}, Y_{\frac{n+1}{2}} \in \mathcal{O}_{v}$ and

$$
Y_{1}, \cdots, Y_{\frac{n-1}{2}} \equiv\left(\begin{array}{ll}
0 & 1 \\
1 & 0
\end{array}\right), \quad Y_{\frac{n+1}{2}} \equiv 1 \quad \bmod \pi_{v} .
$$

Since $Y_{\frac{n+1}{2}} \equiv 1 \bmod \pi_{v}, Y_{\frac{n+1}{2}} \in \mathcal{O}_{v}^{\times}$. By Lemma 11.4, there exists $g_{1} \in$ $\operatorname{GL}(n)_{\mathcal{O}_{v}}$ such that $g_{1} Y=Y_{\frac{n+1}{2}} w_{v, \text { sp }}$. So, if we put $g=\left(Y_{\frac{n+1}{2}}^{-1}, g_{1}\right) \in K_{v}$, then $g Y=w_{v, \mathrm{sp}}$.

Let $H_{v, \text { sp }}$ be the subgroup of $K_{v}$ defined by

$$
H_{v, \mathrm{sp}}=\left\{g \in K_{v} \mid g w_{v, \mathrm{sp}} \in \mathcal{D}_{v, \mathrm{sp}}\right\} .
$$

Clearly $H_{v, \mathrm{sp}} \mathcal{D}_{v, \mathrm{sp}}=\mathcal{D}_{v, \mathrm{sp}}$.

Lemma 11.9. If $n \geq 3$ is odd then

$$
\operatorname{vol}\left(K_{v} w_{v, \mathrm{sp}}\right)=\prod_{j=0}^{\frac{n-1}{2}}\left(1-q_{v}^{-2 j-1}\right)
$$

Proof. Let $\mathcal{A}_{v, \mathrm{sp}} \subseteq K_{v}$ be a set of representatives for $K_{v} / H_{v, \mathrm{sp}}$. Then

$$
K_{v} w_{v, \mathrm{sp}}=\bigsqcup_{h \in \mathcal{A}_{v, \mathrm{sp}}} h \mathcal{D}_{v, \mathrm{sp}}
$$

and $\operatorname{vol}\left(h \mathcal{D}_{v, \mathrm{sp}}\right)=\operatorname{vol}\left(\mathcal{D}_{v, \mathrm{sp}}\right)=q_{v}^{-\frac{n(n+1)}{2}}$ for each $h \in \mathcal{A}_{v, \mathrm{sp}}$.

If $\left(t_{0}, g_{1}\right) \in H_{v, \mathrm{sp}}$, then $g_{1} \bmod \pi_{v}$ is an element of $\mathrm{GO}\left(Q_{n, \mathrm{sp}}\right)_{\mathbb{F}_{v}}$, and $t_{0}$ $\bmod \pi_{v}$ is uniquely determined by $g_{1}$. So

$$
\sharp\left(K_{v} / H_{v, \mathrm{sp}}\right)=\frac{\sharp \mathrm{GL}(1)_{\mathbb{F}_{v}} \times \sharp \mathrm{GL}(n)_{\mathbb{F}_{v}}}{\sharp \mathrm{GO}\left(Q_{n, \mathrm{sp}}\right)_{\mathbb{F}_{v}}}=q^{\frac{n(n+1)}{2}} \prod_{j=0}^{\frac{n-1}{2}}\left(1-q_{v}^{-2 j-1}\right) .
$$

Therefore,

$$
\operatorname{vol}\left(K_{v} w_{v, \mathrm{sp}}\right)=\sharp\left(K_{v} / H_{v, \mathrm{sp}}\right) \times \operatorname{vol}\left(\mathcal{D}_{v, \mathrm{sp}}\right)=\prod_{j=0}^{\frac{n-1}{2}}\left(1-q_{v}^{-2 j-1}\right) .
$$


We next consider $\operatorname{vol}\left(K_{v} w_{v, \mathrm{rm}}\right)$. We define a subset $\mathcal{D}_{v, \mathrm{rm}}$ of $V_{\mathcal{O}_{v}}$ as follows:

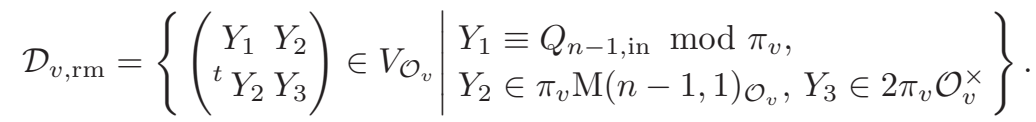

Lemma 11.10. $K_{v} w_{v, \mathrm{rm}}=K_{v} \mathcal{D}_{v, \mathrm{rm}}$.

Proof. Let $Y=\left(\begin{array}{cc}Y_{1} & Y_{2} \\ { }^{t} Y_{2} & Y_{3}\end{array}\right) \in \mathcal{D}_{v, \mathrm{rm}}$. Similarly as in the proof of Lemma 11.6, by applying an element of $K_{v}, Y$ can be made into the following form:

$$
Y=\left(\begin{array}{ll}
Y_{1} & \\
& Y_{2}
\end{array}\right), \quad Y_{1}=\left(\begin{array}{ccc}
Y_{1,1} & & \\
& \ddots & \\
& & Y_{1, \frac{n-1}{2}}
\end{array}\right)
$$

where $Y_{1,1}, \cdots, Y_{1, \frac{n-1}{2}} \in \mathrm{M}(2,2)_{\mathcal{O}_{v}}, Y_{2}=2 \pi_{v} t_{0}^{-1}\left(t_{0} \in \mathcal{O}_{v}^{\times}\right)$and

$$
Y_{1,1}, \cdots, Y_{1, \frac{n-3}{2}} \equiv\left(\begin{array}{ll}
0 & 1 \\
1 & 0
\end{array}\right), Y_{1, \frac{n-1}{2}} \equiv\left(\begin{array}{cc}
2 & a_{0} \\
a_{0} & 2 b_{0}
\end{array}\right) \quad \bmod \pi_{v}
$$

By Lemma 11.4, there exists $g_{1} \in \mathrm{GL}(n-1) \mathcal{O}_{v}$ such that $g_{1} Y_{1}=t_{0}^{-1} w_{n-1, v \text {,in }}$. So, if we put $g=\left(t_{0},\left(\begin{array}{ll}g_{1} & \end{array}\right)\right) \in K_{v}$, then $g Y=w_{n, v, r m}$.

Let $H_{v, \mathrm{rm}}$ be the following subgroup of $K_{v}$ :

$$
\left\{\left(t_{0}, g_{1}\right) \in K_{v} \mid g_{1}=\left(\begin{array}{cc}
h_{11} & h_{12} \\
\pi_{v} h_{21} h_{22}
\end{array}\right), \begin{array}{l}
h_{11} \in \mathrm{GL}(n-1) \mathcal{O}_{v}, h_{22} \in \mathcal{O}_{v}^{\times}, \\
h_{12},{ }^{t} h_{21} \in \mathrm{M}(n-1,1)_{\mathcal{O}_{v}}, \\
t_{0} h_{11} Q_{n-1, \text { in }} \equiv Q_{n-1, \text { in }} \bmod \pi_{v}
\end{array}\right\} .
$$

Since $\left(1, I_{n}\right) \in H_{v, \mathrm{rm}}, H_{v, \mathrm{rm}} \mathcal{D}_{v, \mathrm{rm}} \supseteq \mathcal{D}_{v, \mathrm{rm}}$. Suppose that $g=\left(t_{0}, g_{1}\right) \in H_{v, \mathrm{rm}}$ and $Y \in \mathcal{D}_{v, \text { rm }}$. Let $g_{1}$ be as above. Using block matrices, we express $Y$ and $g Y$ as follows:

$$
\left.Y=\begin{array}{l}
n-1 \\
1
\end{array}\left(\begin{array}{cc}
Y_{1} & Y_{2} \\
{ }^{t} Y_{2} & Y_{3}
\end{array}\right), g Y={ }_{1}^{n-1}{ }^{n-1} \quad \begin{array}{cc}
Y_{1}^{\prime} & Y_{2}^{\prime} \\
{ }^{t} Y_{2}^{\prime} & Y_{3}^{\prime}
\end{array}\right)
$$

It is easy to see that $Y_{1}^{\prime} \equiv Q_{n-1, i n} \bmod \pi_{v}$ and $Y_{2}^{\prime} \equiv 0 \bmod \pi_{v}$. We consider

$$
Y_{3}^{\prime}=t_{0}\left(\pi_{v}^{2} h_{21} Y_{1}{ }^{t} h_{21}+\pi_{v} h_{22}{ }^{t} Y_{2}{ }^{t} h_{21}+\pi_{v} h_{21} Y_{2}{ }^{t} h_{22}+h_{22} Y_{3}{ }^{t} h_{22}\right) \text {. }
$$

Since all diagonal entries of $Y_{1}$ are elements of $2 \mathcal{O}_{v}, t_{0} \pi_{v}^{2} h_{21} Y_{1}^{t} h_{21} \in 2 \pi_{v}^{2} \mathcal{O}_{v}$. Since $Y_{2} \in \pi_{v} \mathrm{M}(n-1,1) \mathcal{O}_{v}$ and $\pi_{v} h_{22}{ }^{t} Y_{2}{ }^{t} h_{21}=\pi_{v} h_{21} Y_{2}{ }^{t} h_{22}, \pi_{v} h_{22}{ }^{t} Y_{2}{ }^{t} h_{21}+$ $\pi_{v} h_{21} Y_{2}{ }^{t} h_{22} \in 2 \pi_{v}^{2} \mathcal{O}_{v}$. Therefore, $Y_{3}^{\prime} \in 2 \pi_{v} \mathcal{O}_{v}^{\times}$. Thus, $H_{v, \mathrm{rm}} \mathcal{D}_{v, \mathrm{rm}}=\mathcal{D}_{v, \mathrm{rm}}$. 
Lemma 11.13. Let $Y_{1}, Y_{2} \in \mathcal{D}_{v, \mathrm{rm}}$. Suppose that $g \in K_{v}$ satisfies $g Y_{1} \equiv Y_{2} \quad \bmod \pi_{v}$. Then $g \in H_{v, \mathrm{rm}}$.

Proof. Let $g=\left(t_{0}, g_{1}\right)$. Using block matrices, we express $g_{1} \in K_{v}$ as follows:

$$
g_{1}=\begin{array}{ll}
n-1 \\
1
\end{array}\left(\begin{array}{ll}
g_{11} & g_{12} \\
g_{21} & g_{22}
\end{array}\right) .
$$

If we express $Y, Y^{\prime}=g Y \in \mathcal{D}_{v, \text { rm }}$ similarly as (11.12), then

$$
\begin{aligned}
& t_{0}^{-1} Y_{1}^{\prime}=g_{11} Y_{1}^{t} g_{11}+g_{12}{ }^{t} Y_{2}{ }^{t} g_{11}+g_{11} Y_{2}{ }^{t} g_{12}+g_{12} Y_{3}{ }^{t} g_{12}, \\
& t_{0}^{-1} Y_{2}^{\prime}=g_{11} Y_{1}^{t} g_{21}+g_{12}{ }^{t} Y_{2}^{t} g_{21}+g_{11} Y_{2}{ }^{t} g_{22}+g_{12} Y_{3}{ }^{t} g_{22}, \\
& t_{0}^{-1} Y_{3}^{\prime}=g_{21} Y_{1}^{t} g_{21}+g_{22}{ }^{t} Y_{2}^{t} g_{21}+g_{21} Y_{2}{ }^{t} g_{22}+g_{22} Y_{3}{ }^{t} g_{22} .
\end{aligned}
$$

Since $Y_{2} \in \pi_{v} \mathrm{M}(n-1,1)_{\mathcal{O}_{v}}$ and $Y_{3} \in 2 \pi_{v} \mathcal{O}_{v}^{\times}, t_{0}^{-1} Y_{1}^{\prime} \equiv g_{11} Y_{1}^{t} g_{11} \bmod \pi_{v}$. Since $Y_{1}, Y_{1}^{\prime} \equiv Q_{n-1, \text { in }} \bmod \pi_{v}$,

$$
t_{0} g_{11} Q_{n-1, \text { in }} \equiv Q_{n-1, \text { in }} \bmod \pi_{v} .
$$

Since $t_{0}^{-1} Y_{2}^{\prime} \in \pi_{v} \mathrm{M}(n-1,1)_{\mathcal{O}_{v}}$,

$$
g_{11} Y_{1}{ }^{t} g_{21}+g_{12}{ }^{t} Y_{2}{ }^{t} g_{21}+g_{11} Y_{2}{ }^{t} g_{22}+g_{12} Y_{3}{ }^{t} g_{22} \in \pi_{v} \mathrm{M}(n-1,1)_{\mathcal{O}_{v}} .
$$

Therefore, $g_{11} Y_{1}{ }^{t} g_{21} \in \pi_{v} \mathrm{M}(n-1,1)_{\mathcal{O}_{v}}$ because $Y_{2} \in \pi_{v} \mathrm{M}(n-1,1)_{\mathcal{O}_{v}}$ and $Y_{3} \in 2 \pi_{v} \mathcal{O}_{v}^{\times}$. Since $g_{11} Y_{1} \in \mathrm{GL}(n-1)_{\mathcal{O}_{v}},{ }^{t} g_{21} \in \pi_{v} \mathrm{M}(n-1,1)_{\mathcal{O}_{v}}$. Since $g_{1} \in \mathrm{GL}(n)_{\mathcal{O}_{v}}, g_{22} \in \mathcal{O}_{v}^{\times}$.

Lemma 11.15. If $n \geq 3$ is odd then

$$
\operatorname{vol}\left(K_{v} w_{v, \mathrm{rm}}\right)=2^{-1} q_{v}^{-1}\left(1-q_{v}^{-\frac{n-1}{2}}\right) \prod_{j=0}^{\frac{n-1}{2}}\left(1-q_{v}^{-2 j-1}\right) .
$$

Proof. Let $\mathcal{A}_{v, \mathrm{rm}} \subseteq K_{v}$ be a set of representatives for $K_{v} / H_{v, \mathrm{rm}}$. By Lemma $11.13, K_{v} w_{v, \mathrm{rm}}=\bigsqcup_{h \in \mathcal{A}_{v, \mathrm{rm}}} h \mathcal{D}_{v, \mathrm{rm}}$. It is easy to see that

$$
\begin{aligned}
\operatorname{vol}\left(h \mathcal{D}_{v, \mathrm{rm}}\right) & =\operatorname{vol}\left(\mathcal{D}_{v, \mathrm{rm}}\right)=q_{v}^{-\frac{n(n-1)}{2}} \times q_{v}^{-(n-1)} \times q_{v}^{-1}\left(1-q_{v}^{-1}\right) \\
& =q_{v}^{-\frac{n(n+1)}{2}}\left(1-q_{v}^{-1}\right)
\end{aligned}
$$


for each $h \in \mathcal{A}_{\text {rm }}$. Since

$$
\begin{aligned}
& \sharp\left(K_{v} / H_{v, \mathrm{rm}}\right)=\frac{\sharp \mathrm{GL}(1)_{\mathbb{F}_{v}} \times \sharp \mathrm{GL}(n)_{\mathbb{F}_{v}}}{\sharp \mathrm{GO}\left(Q_{n-1, \mathrm{in}}\right)_{\mathbb{F}_{v}} \times q_{v}^{n-1} \times \sharp \mathrm{GL}(1)_{\mathbb{F}_{v}}} \\
& =2^{-1} q^{\frac{n(n+1)}{2}-1}\left(1-q_{v}^{-1}\right)^{-1}\left(1-q_{v}^{-\frac{n-1}{2}}\right) \prod_{j=0}^{\frac{n-1}{2}}\left(1-q_{v}^{-2 j-1}\right),
\end{aligned}
$$

we obtain

$$
\begin{aligned}
\operatorname{vol}\left(K_{v} w_{v, \mathrm{rm}}\right) & =\sharp\left(K_{v} / H_{v, \mathrm{rm}}\right) \times \operatorname{vol}\left(\mathcal{D}_{v, \mathrm{rm}}\right) \\
& =2^{-1} q_{v}^{-1}\left(1-q_{v}^{-\frac{n-1}{2}}\right) \prod_{j=0}^{\frac{n-1}{2}}\left(1-q_{v}^{-2 j-1}\right) .
\end{aligned}
$$

By (3.5), if $n \geq 3$ is odd then

$$
\left|P\left(w_{v, \mathrm{i}}\right)\right|_{v}=\left|P\left(w_{v, \dot{\mathrm{i}}}^{\prime}\right)\right|_{v}= \begin{cases}1 & \dot{\mathrm{i}}=\mathrm{sp}, \\ q_{v}^{-1} & \dot{\mathrm{i}}=\mathrm{rm} .\end{cases}
$$

By Lemmas 11.1, 11.9 and 11.15, we obtain the following proposition.

Proposition 11.16. Let $v \in \mathfrak{M}_{\mathrm{f}}$ and $w_{v, \mathrm{i}}^{\prime}$ be an alternative orbital representative for $G_{k_{v}} \backslash V_{k_{v}}^{\mathrm{ss}}$. Then

$$
\vec{c}_{v, \mathrm{i}}^{\prime \prime}= \begin{cases}\prod_{i=1}^{\frac{n-1}{2}}\left(1-q_{v}^{-2 i}\right)^{-1} & \dot{\mathrm{i}}=\mathrm{sp}, \\ 2^{-1} q_{v}^{\frac{n-1}{2}}\left(1-q_{v}^{-\frac{n-1}{2}}\right) \prod_{i=1}^{\frac{n-1}{2}}\left(1-q_{v}^{-2 i}\right)^{-1} & \dot{\mathrm{i}}=\mathrm{rm} .\end{cases}
$$

\section{Acknowledgement}

The authors would like to thank the referee for reading the manuscript carefully and pointing out various mistakes.

\section{References}

[1] A. Borel, Linear algebraic groups, Second edition, Springer, New York, 1991.

[2] F. Chamizo and H. Iwaniec, On the Gauss mean-value formula for class number, Nagoya Math. J. 151 (1998), 199-208. 
[3] B. A. Datskovsky, A mean-value theorem for class numbers of quadratic extensions, in A tribute to Emil Grosswald: number theory and related analysis, 179-242, Contemp. Math., 143, Amer. Math. Soc., Providence, RI.

[4] B. Datskovsky and D. J. Wright, Density of discriminants of cubic extensions, J. Reine Angew. Math. 386 (1988), 116-138.

[5] H. Davenport and H. Heilbronn, On the density of discriminants of cubic fields, Bull. London Math. Soc. 1 (1969), 345-348.

[6] - On the density of discriminants of cubic fields. II, Proc. Roy. Soc. London Ser. A 322 (1971), no. 1551, 405-420.

[7] D. Goldfeld and J. Hoffstein, Eisenstein series of $\frac{1}{2}$-integral weight and the mean value of real Dirichlet $L$-series, Invent. Math. 80 (1985), no. 2, 185-208.

[8] G. Pall, The weight of a genus of positive $n$-ary quadratic forms, in Proc. Sympos. Pure Math., Vol. VIII, 95-105, Amer. Math. Soc., Providence, R.I.

[9] N. Hayasaka and A. Yukie, On the density of unnormalized Tamagawa numbers of orthogonal groups II, to appear in Amer. J. Math.

[10] S. Helgason, Differential geometry, Lie groups, and symmetric spaces, Academic Press, New York, 1978.

[11] T. Ibukiyama and H. Saito, On zeta functions associated to symmetric matrices. I. An explicit form of zeta functions, Amer. J. Math. 117 (1995), no. 5, 1097-1155.

[12] J. Igusa, An introduction to the theory of local zeta functions, Amer. Math. Soc., Providence, RI, 2000.

[13] A. C. Kable and A. Yukie, Prehomogeneous vector spaces and field extensions. II, Invent. Math. 130 (1997), no. 2, 315-344.

[14] - The mean value of the product of class numbers of paired quadratic fields. I, Tohoku Math. J. (2) 54 (2002), no. 4, 513-565.

[15] - The mean value of the product of class numbers of paired quadratic fields. II, J. Math. Soc. Japan 55 (2003), no. 3, 739-764.

[16] J. Number Theory 99 (2003), no. 1, 185-218.

[17] $\longrightarrow$ On the space of quadruples of quinary alternating forms, J. Pure Appl. Algebra 186 (2004), no. 3, 277-295.

[18] R. Lipschutz, in Sitzungsber., pp. 174-185, Akad. Berlin, 1865.

[19] F. Mertens, Über einige asymptotische Gesetze der Zahlentheorie, J. Math. 77 (1874), $312-319$.

[20] D. Mumford, Lectures on curves on an algebraic surface, Princeton Univ. Press, Princeton, N.J., 1966.

[21] W. Narkiewicz, Elementary and analytic theory of algebraic numbers, PWN, Warsaw, 1974.

[22] O. T. O'Meara, Introduction to quadratic forms, Reprint of the 1973 edition, Springer, Berlin, 2000.

[23] V. Platonov and A. Rapinchuk, Algebraic groups and number theory, Translated from the 1991 Russian original by Rachel Rowen, Academic Press, Boston, MA, 1994.

[24] I. Satake, Theory of spherical functions on reductive algebraic groups over $\mathfrak{p}$-adic fields, Inst. Hautes Études Sci. Publ. Math. No. 18 (1963), 5-69.

[25] T. Shintani, On Dirichlet series whose coefficients are class numbers of integral binary cubic forms, J. Math. Soc. Japan 24 (1972), 132-188.

[26] _ On zeta-functions associated with the vector space of quadratic forms, J. Fac. Sci. Univ. Tokyo Sect. I A Math. 22 (1975), 25-65.

[27] C. L. Siegel, The average measure of quadratic forms with given determinant and signature, Ann. of Math. (2) 45 (1944), 667-685.

[28] T. Tamagawa, Adèles, in Algebraic Groups and Discontinuous Subgroups (Proc. Sympos. Pure Math., Boulder, Colo., 1965), 113-121, Amer. Math. Soc., Providence, R.I.

[29] I. M. Vinogradov, Selected works, Translated from the Russian by Naidu Psv [P. S. V. Naidu], Translation edited by Yu. A. Bakhturin, Springer, Berlin, 1985. 
[30] G. L. Watson, The 2-adic density of a quadratic form, Mathematika 23 (1976), no. 1, 94-106.

[31] A. Weil, Basic number theory, Third edition, Springer, New York, 1974

[32] Adeles and algebraic groups, Birkhäuser, Boston, Mass., 1982.

[33] D. J. Wright and A. Yukie, Prehomogeneous vector spaces and field extensions, Invent. Math. 110 (1992), no. 2, 283-314.

[34] A. Yukie, On the density of unnormalized Tamagawa numbers of orthogonal groups III, preprint.

[35] Shintani zeta functions, Cambridge Univ. Press, Cambridge, 1993.

[36] On the Shintani zeta function for the space of binary tri-Hermitian forms, Math. Ann. 307 (1997), no. 2, 325-339.

[37] On the Shintani zeta function for the space of pairs of binary Hermitian forms, J. Number Theory 92 (2002), no. 2, 205-256. 